\title{
The Wave Equation: Control and Numerics*
}

\author{
Sylvain Ervedoza and Enrique Zuazua
}

\begin{abstract}
In these Notes we make a self-contained presentation of the theory that has been developed recently for the numerical analysis of the controllability properties of wave propagation phenomena and, in particular, for the constant coefficient wave equation. We develop the so-called discrete approach. In other words, we analyze to which extent the semidiscrete or fully discrete dynamics arising when discretizing the wave equation by means of the most classical scheme of numerical analysis, shear the property of being controllable, uniformly with respect to the mesh-size parameters and if the corresponding controls converge to the continuous ones as the mesh-size tends to zero. We focus mainly on finite-difference approximation schemes for the one-dimensional constant coefficient wave equation. Using the well known equivalence of the control problem with the observation one, we analyze carefully the second one, which consists in determining the total energy of solutions out of partial measurements. We show how spectral analysis and the theory of non-harmonic Fourier series allows, first, to show that high frequency wave packets may behave in a pathological manner and, second, to design efficient filtering mechanisms. We also develop the multiplier approach that allows to provide energy identities relating the total energy of solutions and the energy concentrated
\end{abstract}

Sylvain Ervedoza

CNRS ; Institut de Mathématiques de Toulouse UMR 5219 F-31062 Toulouse, France, Université de Toulouse ; UPS, INSA, INP, ISAE, UT1, UTM ; IMT F-31062 Toulouse, France e-mail: sylvain.ervedoza@math.univ-toulouse.fr

Enrique Zuazua

BCAM - Basque Center for Applied Mathematics, Bizkaia Technology Park, B.500, E48160 Derio, Basque Country, Spain.

Ikerbasque Research Professor, Ikerbasque - Basque Foundation for Science, E48011 Bilbao, Basque Country, Spain.

e-mail: zuazua@bcamath.org.

* This work was supported by the ERC Advanced Grant FP7-246775 NUMERIWAVES, the Grant PI2010-04 of the Basque Government, the ESF Research Networking Program OPTPDE and Grant MTM2008-03541 of the MICINN, Spain. The first author acknowledges the hospitality and support of the Basque Center for Applied Mathematics where part of this work was done. 
on the boundary. This observability properties obtained after filtering, by duality, allow to build controls that, normally, do not control the full dynamics of the system but rather guarantee a relaxed controllability property. Despite of this they converge to the continuous ones. We also present a minor variant of the classical Hilbert Uniqueness Method allowing to build smooth controls for smooth data. This result plays a key role in the proof of the convergence rates of the discrete controls towards the continuous ones. These results are illustrated by means of several numerical experiments.

\section{Introduction}

In these notes, we make a survey presentation of the work done in the last years on the problems of controllability and observability of waves from a numerical analysis viewpoint. In particular, we explain that, even for numerical schemes that converge in the classical sense of numerical analysis, one cannot expect them to automatically be well behaved for observation and control purposes. This paper is essentially an updated version of [114], in which we collect most of the more recent developments.

Problems of control and observation of waves arise in many different contexts and for various models but, to be more precise and better present the milestones of the theory that has been developed so far, we will focus our analysis on the wave equation, and mainly in the 1-dimensional setting where several methods can be used to get rather explicit and complete results. We shall mainly focus on the finite difference method on a regular grid. Some of these results can be extended to several space dimensions but, still, a lot remains to be done to deal with general variable coefficient wave equations and with schemes on non-uniform grids in one and several space dimensions.

Controllability refers to the possibility of driving the system under consideration (here, the wave equation) to a prescribed final state at a given final time using a control function. Of course, this question is interesting when the control function does not act everywhere but is rather located in some part of the domain or on its boundary through suitable actuators.

On the other hand, observability refers to the possibility of measuring the whole energy of the solutions of the free trajectories (i.e., without control) through partial measurements. Again, one easily understands that such a property is interesting and non-trivial only when the measurements are not complete and done on the whole domain where waves propagate, but they are rather localized in part of the domain or on its boundary through suitable sensors.

It turns out that these two properties are equivalent and dual one from another. This is the basis of the so-called Hilbert Uniqueness Method [68, 69] introduced by J.-L. Lions, that we shall recall more precisely in Section 2, first in a finite dimensional setting and then for abstract conservative systems such as the wave equation.

In particular, on the basis of the Hilbert Uniqueness Method (HUM) one can also build algorithms to compute the optimal control, the one of of minimal norm, in a 
sense to be made precise (see details in Section 2). We shall in particular explain how, using the observability property, one can slightly modify HUM with a weight function in time, vanishing for the initial and final time, so that the control obtained minimizing this functional preserves the regularity properties of the data to be controlled, see Section 2.3 and [35]. Curiously enough, these results, inspired in [24] where the regularity of the control for the wave equation is analyzed through microlocal analysis, are very recent. The abstract version of these results in [35], was proved using a simplified proof without requiring microlocal analysis tools. Note however that the results in [24], which are specific to the wave equation, are stronger than the ones in [35] since they yield also a very precise dyadic decomposition of the controls.

In the context of wave propagation phenomena, observability and controllability properties are very much related to the propagation of rays, that, for the constant coefficient wave equation, are straight space-time lines traveling at velocity one and bouncing on the boundary according to the Descartes-Snell law of Geometric Optics (see Section 3.4 and [6], [14]). In view of the finite velocity of propagation of rays, as we shall explain, one needs the observation/control time to be large enough to allow all the characteristics to meet the observation/control region and ensure the observability/controllability properties to hold.

In 1-d, these properties of propagation and reflection are the essence of the method of characteristics leading to D'Alembert's formula. That is why our presentation of the observability/controllability of waves focuses mainly in the 1-d setting, see Section 3

As we said above, HUM characterizes the optimal control through a minimization process of a quadratic coercive functional for the solutions of the adjoint wave equation. This allows characterizing the controls through the corresponding EulerLagrange equations or Optimality System and building efficient algorithms for computing them. This is the so called continuous approach in which one first derives a complete characterization of the controls for the continuous wave model to later use numerical analysis tools to approximate them. There are of course different ways of implementing this continuous approach for the construction of numerical approximations of the controls. The first article devoted to this issue is, probably, [5]. Recently, the continuous approach to numerical control was developed differently by Cindae et al. [20]. They adapted at the numerical level the well-known iterative algorithm by D. Russell [96] in which the property of controllability is obtained from the stabilization one by an iterated back and forth application of the dissipative semigroup. We also refer to the recent works of D. Auroux et J. Blum [3] that have developed a similar approach in the context of the control of nonlinear viscous conservation laws. As we shall further explain in [32], the methods in [20] lead to very similar algorithms to those one would get by applying numerical approximations in the conjugate gradient algorithm associated to the continuous minimization problem that HUM leads to. While in [20] the back and forth iteration is done always on the dissipative system, when following HUM one alternates between the state equation and the adjoint one in dual functional settings. Both approaches lead to similar convergence rates but the HUM one is more flexible since it can be adapted to a large 
class of problems, including those in which the control operator is unbounded as it happens often in practice and in particular for the boundary control of the wave equation.

But, very often in practice, one frequently applies the more direct, so called, discrete approach which consists on, first, discretizing the equation using a convergent numerical approximation scheme, to later compute a control for this numerical approximation. The model obtained after numerical discretization being a finite dimensional time continuous or discrete system, the computation of its control can be performed using standard existing finite-dimensional methods and software. But such natural approach often fails. In particular, in the context of the wave equation under consideration, as we shall see below, for some initial data, this approach yields discrete controls that are not even bounded as the mesh-size goes to zero, see Theorem 8 .

Note that this point of view was systematically developed by R. Glowinski, J. L. Lions and coworkers (see [43], [44]) to build numerical approximation algorithms. In their works they developed and implement conjugate gradient descent algorithms combined with Finite Element Methods for approximating the wave equations. They observed the bad conditioning of the corresponding discrete problems and indicated the need of filtering the high frequencies. This was done in particular using two-grid filtering techniques (see R. Glowinski [40]) and motivated a substantial part of the work that we present in this article.

Part of this paper is devoted to develop a thorough study of this divergence or blow up phenomenon for the space finite-difference semidiscrete 1-d wave equation as a model example since other classical schemes, such as the ones given by finite element methods, exhibit the same behavior.

Our approach is based on the analysis of the observability property of these finitedimensional systems that approximate the 1-d wave equation. In particular, as we shall see, even when the convergence of the numerical method in the classical sense of numerical analysis is guaranteed, the discrete systems are not uniformly observable with respect to the space discretization parameter, see Theorem 6 As a consequence, by duality, there are initial data for which the sequence of corresponding discrete controls diverge (Theorem 8). In other words, the stability in what concerns the solvability of the initial-boundary value problem is not sufficient to guarantee the stability with respect to the observability property.

The lack of uniform observability can be explained and understood by looking to the propagation properties of the solutions of the numerical approximation schemes. In Section 4.3. we will explain that the numerical schemes generate spurious solutions traveling with the so called group velocity which, for high frequency numerical solutions, is of the order of the mesh-size parameter [104, 101, 72]. To be more precise, the high-frequencies involved in these wave packets are of the order of $1 / h, h$ being the space mesh-size. Asymptotically, as $h$ tends to zero, they weakly converge to zero, thus being compatible with the convergence of the numerical scheme in the classical sense of numerical analysis, while being an obstruction for the observability property to hold uniformly with respect to the mesh size parameter $h$. This is so since the time that these wave packets need to get into the observation region 
is of the order of $1 / h$. Actually, for $T>0$ fixed, the observability constant for the semidiscrete problems is of the order of $\exp (C / h)$, see [77].

Our analysis of the lack of uniform observability for the discrete waves also indicates the path to avoid these divergence and blow up phenomena to occur. A careful analysis of the velocity of propagation of numerical waves shows that low frequency components propagate with a uniform velocity, a fact that is compatible with uniform observability properties. Here, by low-frequencies we refer to those covering a fixed percentage of the spectrum of the corresponding discrete dynamics, independent of the mesh-size. These "low" frequencies end up filling all the frequency range as the discretization parameter goes to zero. This shows how, through filtering, i.e. focusing on the low-frequency components, one can prove uniform observability results and still, by letting $h$ tend to zero, recover the full dynamics of the continuous model. The need of filtering the high-frequency components to focus on the low-frequency ones was already observed in the papers by R. Glowinski, J.-L. Lions \& all [40, 43, 44]. Among the different ways of doing that, in this paper we shall present Fourier filtering techniques [53], Tychnonoff regularization methods and bi-grid techniques [2, 83, 82].

In Section 5 we show how these ideas yield observability properties that hold uniformly with respect to the discretization parameters within the subspace of filtered solutions. We will also briefly present the results in [111] and in [27] in the multi-dimensional setting.

These uniform observability properties lead to controllability results with uniformly bounded controls. However, the controls one obtains in this manner do not control the full state but only suitable low-frequency projections of the numerical solutions. We shall then show how to prove the convergence of the discrete controls towards the continuous ones and to derive convergence rates. The procedure we describe is general and can be adapted to various situations, i. e. different models and numerical approximation schemes.

Note that, to the best of our knowledge, this is the first time that convergence rates are proved. This requires, in particular, a systematic method to build controls preserving the regularity of the data to be controlled. This is done by a suitable weighted version of the HUM-method, see Theorem[3] and [35].

The paper is organized as follows. Section 2 recalls the basic facts on the Hilbert Uniqueness Method. It also includes the main results of [35] on the regularity of controls for smooth data. In Section 3 we present the main observability/controllability results for the constant coefficient one-dimensional (1-d) wave equation, and briefly comment on the works [6, 14] in the multi-dimensional setting, using microlocal analysis techniques. The main results on the lack of observability/controllability of finite difference semidiscretizations are then presented in Section 4 . In Section 5 , we discuss several methods for curing high-frequency pathologies and getting weak observability estimates. In Section 6 we describe how these observability results can be used to develop numerical methods to obtain discrete controls that converge towards the continuous ones, with explicit convergence rates whenever the data to be controlled are smoother. In Section 7 with discuss several other related issues and present a list of interesting open problems. 
Notations

In all these notes, we shall use several different notations:

- In an abstract setting, $x$ is the state, solution of the controlled equation, $A$ is the operator that prescribes the dynamics, $B$ is the control operator, $v$ is a generic control and $\varphi$ is the solution of the adjoint equation.

- When considering the wave equation, the state is denoted by $y$ and the adjoint state by $u$.

- Several controls shall appear. The notation $v$ is used to denote a generic control function. $V_{\text {hum }}$ refers to the control given by the Hilbert Uniqueness Method and $V$ to the control given by the method in [35], which will be explained hereafter in Section 2.1 .

- Indexes $h$ will refer to the space mesh size and all the above notations will be denoted with indexes $h$ when denoting quantities related to the semidiscrete system. Furthermore, all vectorial quantities depending on $h$ are noted in bold characters.

\section{Control and observation of finite-dimensional and abstract systems}

\subsection{Control of Finite-Dimensional Systems}

Numerical approximation schemes and, more precisely, those that are semidiscrete (discrete in space and continuous in time) yield finite-dimensional systems of Ordinary Differential Equations (ODEs).

There is by now an extensive literature on the control of finite-dimensional systems, and the problem is well understood for linear ones (see [66, 98]). In this Section we recall the basics ingredients of the theory and we present it in a manner well suited to be extended to the PDE setting and to the limit process from finite to infinite dimensions that numerical analysis requires (see [78, 36] for more details). Indeed, the problem of convergence of controls as the mesh size in the numerical approximation tends to zero is very closely related to passing to the limit as the dimension of finite-dimensional systems tends to infinity. The latter topic is widely open and this article aims at describing some of its key aspects.

Consider the finite-dimensional system of dimension $N$ :

$$
x^{\prime}=A x+B v, \quad 0 \leq t \leq T ; \quad x(0)=x^{0},
$$

where $x=x(t) \in \mathbb{R}^{N}$ is the $N$-dimensional state and $v=v(t) \in \mathbb{R}^{M}$ is the $M$ dimensional control, with $M \leq N$.

Here $A$ is an $N \times N$ matrix with constant real coefficients and $B$ is an $N \times M$ matrix. The matrix $A$ determines the dynamics of the system and $B$ models the way $M$ controls act on it. 
In practice, it is desirable to control the $N$ components of the system with a low number of controls, and the best would be to do it by a single one, in which case $M=1$.

System (1) is said to be controllable in time $T$ when every initial datum $x^{0} \in \mathbb{R}^{N}$ can be driven to any final datum $x^{T}$ in $\mathbb{R}^{N}$ in time $T$. In other words, we ask if for any $\left(x^{0}, x^{T}\right) \in\left(\mathbb{R}^{N}\right)^{2}$, there exists a control function $v:[0, T] \rightarrow \mathbb{R}^{M}$ so that the solution $x$ of (1) satisfies

$$
x(T)=x^{T} .
$$

Since we are in a linear finite dimensional setting, it is easy to check that system (1) is controllable in time $T>0$ if and only if it is null-controllable in time $T>0$, i.e. if for any $x^{0} \in \mathbb{R}^{N}$, there exists a control function $v:[0, T] \rightarrow \mathbb{R}^{M}$ so that the solution $x$ of (1) satisfies

$$
x(T)=0 .
$$

In the following we shall focus on the null-controllability and we shall refer to it simply as controllability.

There is a necessary and sufficient condition for controllability which is purely algebraic in nature. It is the so-called Kalman condition: System (1) is controllable in some time $T>0$ if and only if

$$
\operatorname{rank}\left[B, A B, \ldots, A^{N-1} B\right]=N .
$$

There is a direct proof of this result which uses the representation of solutions of (11) by means of the variations of constants formula. However, the methods we shall develop along this article rely more on the dual (but completely equivalent!) problem of observability of the adjoint system that we discuss now.

Consider the adjoint system

$$
-\varphi^{\prime}=A^{*} \varphi, \quad 0 \leq t \leq T ; \quad \varphi(T)=\varphi^{T} .
$$

Multiplying (1) by $\varphi$ and integrating it on $(0, T)$, one immediately gets that for all $\varphi^{T} \in \mathbb{R}^{N}$,

$$
0=\int_{0}^{T}\left\langle v, B^{*} \varphi\right\rangle_{\mathbb{R}^{M}} d t+\left\langle x^{0}, \varphi(0)\right\rangle_{\mathbb{R}^{N}} .
$$

Hence $v$ is a control function for (1) if and only if for all $\varphi^{T} \in \mathbb{R}^{N}$,

$$
0=\int_{0}^{T}\left\langle v, B^{*} \varphi\right\rangle_{\mathbb{R}^{M}} d t+\left\langle x^{0}, \varphi(0)\right\rangle_{\mathbb{R}^{N}} .
$$

This characterization of the controls for (1) is the heart of the duality methods we shall use in all these notes, the so-called Hilbert Uniqueness Method (HUM), introduced by J. L. Lions in [68, 69] and that has tremendously influenced the recent development of the field of PDE control and related topics.

Theorem 1. System (1) is controllable in time $T$ if and only if the adjoint system (5) is observable in time $T$, i.e., if there exists a constant $C_{o b s}=C_{o b s}(T)>0$ such that, 
for every solution $\varphi$ of (5) with initial data $\varphi^{T}$ it holds:

$$
|\varphi(0)|_{\mathbb{R}^{N}}^{2} \leq C_{o b s}^{2} \int_{0}^{T}\left|B^{*} \varphi\right|_{\mathbb{R}^{M}}^{2} d t .
$$

Both properties hold in all time $T$ if and only if the Kalman rank condition (4) is satisfied.

Remark 1. The equivalence between the controllability of the state equation and the observability of the adjoint one is one of the most classical ingredients of the controllability theory of finite-dimensional systems (see, for instance, Theorem 1.10.2 in [57]). In general, observability refers to the possibility of recovering the full solution by means of some partial measurements or observations. In the present context, i. e. in (8), one is allowed to measure the output $B^{*} \varphi$ during the time interval $(0, T)$ and wishes to recover complete information on the initial datum $\varphi(0)$. Since in finite-dimensions all norms are equivalent, and the ODEs under consideration are well-posed in the forward and backward sense of time, observing the value of the solution of the adjoint state equation $\varphi(0)$ at $t=0$ as in (8) is equivalent to observing its datum $\varphi^{T}$ at time $t=T$ or both of them.

Proof. We proceed in several steps.

Step 1. Construction of controls as minimizers of a quadratic functional. The proof we present here provides a constructive method for building controls from the observability inequality (8). Indeed, assume (8) holds and consider the quadratic functional $J: \mathbb{R}^{N} \rightarrow \mathbb{R}:$

$$
J\left(\varphi^{T}\right)=\frac{1}{2} \int_{0}^{T}\left|B^{*} \varphi(t)\right|_{\mathbb{R}^{M}}^{2} d t+\left\langle x^{0}, \varphi(0)\right\rangle_{\mathbb{R}^{N}} .
$$

If $\Phi^{T}$ is a minimizer for $J$, since $D J\left(\Phi^{T}\right)=0$, then the control

$$
V_{\text {hum }}=B^{*} \Phi,
$$

where $\Phi$ is the solution of (5) with initial datum $\Phi^{T}$ at time $t=T$ satisfies (7). Hence the corresponding solution $x$ of (1) satisfies the control requirement $x(T)=0$.

Thus, to build the control it is sufficient to minimize the functional $J$. For, we apply the direct method of the calculus of variations. The functional $J$ being continuous, quadratic, and nonnegative, since we are in finite space dimensions, it is sufficient to prove its coercivity, which holds if and only if (8) holds.

Step 2. Equivalence between the observability inequality (8) and the Kalman condition.

Since we are in finite-dimensions and all norms are equivalent, (8) is equivalent to the following uniqueness or unique continuation property: Does the fact that $B^{*} \varphi$ vanish for all $0 \leq t \leq T$ imply that $\varphi \equiv 0$ ?

Taking into account that solutions $\varphi$ are analytic in time, $B^{*} \varphi$ vanishes for all $t \in$ $(0, T)$ if and only if all the derivatives of $B^{*} \varphi$ of any order at time $t=T$ vanish. Since $\varphi=e^{-A^{*}(t-T)} \varphi^{T}$ this is equivalent to $B^{*}\left[A^{*}\right]^{k} \varphi^{T} \equiv 0$ for all $k \geq 0$. But, according 
to the Cayley-Hamilton theorem, this holds if and only if it is satisfied for all $k=$ $0, \ldots, N-1$. Therefore $B^{*} \varphi \equiv 0$ is equivalent to $\left.\varphi^{T} \in \cap_{k \in\{0, N-1\}} \operatorname{Ker}\left(B^{*}\left[A^{*}\right]^{k}\right]\right)$. Hence (8) holds if and only if $\left.\cap_{k \in\{0, \cdots, N-1\}} \operatorname{Ker}\left(B^{*}\left[A^{*}\right]^{k}\right]\right)=\{0\}$, which is obviously equivalent to (4).

Step 3. Lack of controllability when unique continuation fails. If the observability estimate (8) does not hold, there exists a non-trivial $\hat{\varphi}^{T} \neq 0$ so that $B^{*} \hat{\varphi}(t)=0$ for all $t \in(0, T)$. We claim that the initial data $x^{0}=\hat{\varphi}(0)$ cannot be steered to $x^{T}=0$. Otherwise, for some control function $v$, one would have from (7) that

$$
0=\int_{0}^{T}\left\langle v, B^{*} \hat{\varphi}\right\rangle_{\mathbb{R}^{M}}+\left\langle x^{0}, \hat{\varphi}(0)\right\rangle_{\mathbb{R}^{N}},
$$

which would imply $|\hat{\varphi}(0)|^{2}=0$ and then contradict the fact that $\hat{\varphi}^{T} \neq 0$.

Remark 2. The problem of observability can be formulated as that of determining uniquely the adjoint state everywhere in terms of partial measurements. The property of observability of the adjoint system (5) is equivalent to the inequality (8) because of the linear character of the system. In the context of infinite-dimensional systems or PDE this issue is sensitive to the norms under consideration.

Remark 3. It is important to note that in this finite-dimensional context, the value of time $T$ plays no role in what concerns the property of controllability. In particular, whether a system is controllable (or its adjoint observable) is independent of the time $T$ of control. Note that the situation is totally different for the wave equation. There, due to the finite velocity of propagation, the time needed to control/observe waves from the boundary needs to be large enough, of the order of the size of the ratio between the size of the domain and the velocity of propagation.

In fact, the main task to be undertaken to pass to the limit in numerical approximations of control problems for wave equations as the mesh size tends to zero is to explain why, even though at the finite-dimensional level the control time $T$ is irrelevant, it may play a key role for PDEs.

Note however that, even at the level of finite-dimensional systems, the problem of how the size of controls depend on the control time $T$ and in particular how they behave as $T \rightarrow 0$ is an interesting issue, see [97].

Remark 4. Using (7) with $\varphi=\Phi$ given by the minimization of the functional $J$ in (9), one easily checks that any control for (1)-(3) satisfies

$$
\begin{aligned}
\int_{0}^{T}\left\langle v, V_{\text {hum }}\right\rangle_{\mathbb{R}^{M}} d t=\int_{0}^{T}\left\langle v, B^{*} \Phi\right\rangle_{\mathbb{R}^{M}} d t & =-\left\langle x^{0}, \Phi(0)\right\rangle_{\mathbb{R}^{N}} \\
& =\int_{0}^{T}\left|B^{*} \Phi\right|_{\mathbb{R}^{M}}^{2} d t=\int_{0}^{T}\left|V_{\text {hum }}\right|_{\mathbb{R}^{M}}^{2} d t
\end{aligned}
$$

This immediately yields that the HUM control $V_{\text {hum }}$ is the one of minimal $L^{2}\left(0, T ; \mathbb{R}^{M}\right)$ norm.

The proof of Theorem 1 also yields the following important result: 
Corollary 1. Given $T>0$, we assume that $(8)$ holds.

Then for any $x^{0} \in \mathbb{R}^{N}$, there is only one control function satisfying (3) that can be written as $B^{*} \varphi$ for $\varphi$ solution of (5). This is the so-called HUM control $V_{\text {hum }}$ constructed in (9)-(10).

Proof. Such a control should satisfy $\mathbf{7 7}$, hence $\varphi(T)$ should be a critical point of $J$ defined in (96). But $J$ is strictly convex because of (8) and therefore has only one critical point.

Again, this has an important consequence:

Corollary 2. Given $T>0$, we assume that (8) holds.

Then the map constructed in Theorem 1

$$
\mathbb{V}_{\text {hum }}: x^{0} \in \mathbb{R}^{N} \mapsto V_{\text {hum }} \in L^{2}\left(0, T ; \mathbb{R}^{M}\right),
$$

where $V_{\text {hum }}$ is the control computed in [10, is linear.

Proof. Given any pairs $x_{1}^{0}, x_{2}^{0}$, obviously, by linearity, the solution $x$ of 11 with initial data $\left(x_{1}^{0}+\lambda x_{2}^{0}\right)$ and control function $\mathbb{V}_{\text {hum }}\left(x_{1}^{0}\right)+\lambda \mathbb{V}_{\text {hum }}\left(x_{2}^{0}\right)$ satisfies $x(T)=0$. Moreover, using Corollary (1), one easily deduces that $\mathbb{V}_{\text {hum }}\left(x_{1}^{0}+\lambda x_{2}^{0}\right)$ coincides with $\mathbb{V}_{\text {hum }}\left(x_{1}^{0}\right)+\lambda \mathbb{V}_{\text {hum }}\left(x_{2}^{0}\right)$.

The norm of this map can even be characterized:

Theorem 2. Given $T>0$, we assume that (8) holds, the norm of the control map $\mathbb{V}_{\text {hum }}: \mathbb{R}^{N} \rightarrow L^{2}\left(0, T ; \mathbb{R}^{M}\right)$ coincides with $C_{\text {obs }}$, the observability constant in $\mathbb{8}$.

Proof. The proof of the controllability in Theorem 1 yields explicit bounds on the controls $V_{\text {hum }}$ in (10) in terms of the observability constant in (8). Indeed, plugging $\varphi=\Phi$ in (7), the control $V_{\text {Hum }}$ given by (10) can be seen to satisfy

$$
\left\|V_{\text {hum }}\right\|_{L^{2}\left(0, T ; \mathbb{R}^{M}\right)} \leq C_{\text {obs }}\left|x^{0}\right|_{\mathbb{R}^{N}},
$$

$C_{o b s}$ being the same constant as in $(8)$. Therefore, $\left\|\mathbb{V}_{h u m}\right\|_{\mathfrak{L}\left(\left(\mathbb{R}^{N}\right)^{2} ; L^{2}\left(0, T ; \mathbb{R}^{M}\right)\right)} \leq C_{o b s}$. We shall now prove the reverse inequality. Take $\hat{\varphi}$ non-trivial such that it saturates (8), and set $x^{0}=-\hat{\varphi}(0)$. Then, using (7) with $\varphi=\hat{\varphi}$, any control $v$ for $x^{0}$ should satisfy

$$
|\hat{\varphi}(0)|_{\mathbb{R}^{N}}^{2}=\left|x^{0}\right|_{\mathbb{R}^{N}}^{2}=\int_{0}^{T}\left\langle v, B^{*} \hat{\varphi}\right\rangle_{\mathbb{R}^{M}} d t \leq\|v\|_{L^{2}\left(0, T ; \mathbb{R}^{M}\right)}\left\|B^{*} \hat{\varphi}\right\|_{L^{2}\left(0, T ; \mathbb{R}^{M}\right)} .
$$

Using that $\hat{\varphi}$ is non-trivial and saturates 8 , we find out that the control function $\mathbb{V}_{\text {hum }}(-\hat{\varphi}(0))$ should be of norm at least $C_{o b s}|\hat{\varphi}(0)|$, hence the result.

Remark 5. Step 3 of the proof of Theorem 1 and the proof of Theorem 2 rely on the same idea, that data that are difficult to observe correspond to the ones that are the most difficult to control. 


\subsection{Controllability and observability for abstract conservative systems}

In this Section, let $X$ be a Hilbert space endowed with the norm $\|\cdot\|_{X}$ and let $\mathbb{T}=$ $\left(\mathbb{T}_{t}\right)_{t \in \mathbb{R}}$ be a strongly continuous group on $X$, with generator $A: \mathscr{D}(A) \subset X \rightarrow X$.

We further assume that $A$ is a skew-adjoint operator $A^{*}=-A$.

For convenience, we also assume that $A$ is invertible with continuous inverse in $X$. This can be done without loss of generality by translating the semigroup if necessary using $\beta \in \mathbb{R}$ and replacing $A$ by $A-i \beta I$.

Define then the Hilbert space $X_{1}=\mathscr{D}(A)$ of elements of $X$ such that $\|A x\|_{X}<\infty$, endowed with the norm $\|\cdot\|_{1}=\|A \cdot\|_{X}$. Also define $X_{-1}$ as the completion of $X$ with respect to the norm $\|\cdot\|_{-1}=\left\|A^{-1} \cdot\right\|_{X}$.

Let us then consider the control system

$$
x^{\prime}=A x+B v, \quad t \geq 0, \quad x(0)=x^{0} \in X,
$$

where $B \in \mathfrak{L}\left(\mathscr{U}, X_{-1}\right), \mathscr{U}$ is an Hilbert space which describes the possible actions of the control, and $v \in L_{l o c}^{2}([0, \infty) ; \mathscr{U})$ is a control function.

We assume that the operator $B$ is admissible in the sense of [102, Def. 4.2.1]:

Definition 1. The operator $B \in \mathfrak{L}\left(\mathscr{U}, X_{-1}\right)$ is said to be an admissible control operator for $\mathbb{T}$ if for some $\tau>0$, the operator $\mathscr{R}_{\tau}$ defined on $L^{2}(0, T ; \mathscr{U})$ by

$$
\mathscr{R}_{\tau} v=\int_{0}^{\tau} \mathbb{T}_{\tau-s} B v(s) d s
$$

satisfies $\operatorname{Ran} \mathscr{R}_{\tau} \subset X$, where $\operatorname{Ran} \Phi_{\tau}$ denotes the range of the map $\Phi_{\tau}$.

When $B$ is an admissible control operator for $\mathbb{T}$, system (13) is called admissible.

Note that, obviously, if $B$ is a bounded operator, that is if $B \in \mathfrak{L}(\mathscr{U}, X)$, then $B$ is admissible for $\mathbb{T}$. But there are non-trivial examples as, for instance, the boundary control of the wave equation with Dirichlet boundary conditions, in which $B$ is unbounded but admissible, see [68]. In such cases, the admissibility property is then a consequence of a suitable hidden regularity result for the solutions of the adjoint system.

To be more precise, the admissibility of $B$ for $\mathbb{T}$ is equivalent to the existence of a time $T>0$ and a constant $K_{T}>0$ such that any solution of

$$
\varphi^{\prime}=A \varphi, \quad t \in(0, T), \quad \varphi(T)=\varphi^{T}
$$

satisfies

$$
\int_{0}^{T}\left\|B^{*} \varphi(t)\right\|_{\mathscr{U}}^{2} d t \leq K_{T}\left\|\varphi^{T}\right\|_{X}^{2}
$$

In this Section we will always assume that $B$ is an admissible control operator for $\mathbb{T}$. Then, for every $x^{0} \in X$ and $v \in L_{l o c}^{2}([0, \infty) ; \mathscr{U})$, equation (13) has a unique mild solution $x$ which belongs to $C([0, \infty) ; X)$ (see [102, Prop. 4.2.5]). 
Our purpose is to study the controllability of system (13).

System (13) is said to be null controllable in time $T$ if for any $x^{0} \in X$, there exists a control function $v \in L^{2}(0, T ; \mathscr{U})$ such that the solution of 13 satisfies

$$
x(T)=0 .
$$

System (13) is said to be null controllable if it is null controllable in some time $T>0$.

Note that since system (13) is linear and time-reversible, an easy argument left to the reader shows that system (13) is null controllable in time $T$ if and only if it is exactly controllable, i.e. for all $x^{0}, x^{T}$ in $X$, there exists a control function $v \in$ $L^{2}(0, T ; \mathscr{U})$ such that the solution $x$ of 13 with initial data $x^{0}$ and control function $v$ satisfies $x(T)=x^{T}$. Hence we will focus on the null-controllability property in the sequel, and we shall refer to it simply as controllability.

Here again, we claim that system (13) is controllable in time $T$ if and only if there exists a constant $C_{o b s}$ such that all solutions $\varphi$ of the adjoint equation (14) satisfy

$$
\|\varphi(0)\|_{X}^{2} \leq C_{o b s}^{2} \int_{0}^{T}\left\|B^{*} \varphi(t)\right\|_{\mathscr{U}}^{2} d t
$$

We shall refer the interested reader to [68] for the proof of the fact that the exact controllability in time $T$ implies the observability (17) for the adjoint system (14). This is based on a closed graph theorem.

The other implication is actually proved in Step 1 of the proof of Theorem 1 , which describes the Hilbert Uniqueness Method. The idea is to find a minimizer of the functional

$$
J\left(\varphi^{T}\right)=\frac{1}{2} \int_{0}^{T}\left\|B^{*} \varphi(t)\right\|_{\mathscr{U}}^{2} d t+\left\langle x^{0}, \varphi(0)\right\rangle_{X}, \quad \varphi^{T} \in X .
$$

Note that such a minimizer exists and is unique due to the observability property (17). Then, if $\Phi^{T}$ denotes the minimizer of $J$, since $D J\left(\Phi^{T}\right)=0$, the function

$$
V_{\text {hum }}=B^{*} \Phi,
$$

where $\Phi$ is the corresponding solution of (14) is a control function. Indeed, it satisfies for all $\varphi^{T} \in X$,

$$
\int_{0}^{T}\left\langle V_{\text {hum }}, B^{*} \varphi(t)\right\rangle_{\mathscr{U}} d t+\left\langle x^{0}, \varphi(0)\right\rangle_{X}=0
$$

which, as in (7), characterizes the controls of (13). 


\subsection{Smoothness results for HUM controls}

In this Section, we assume that the adjoint system (14) satisfies the observability assumption (17) in some time $T^{*}$. We also assume that the admissibility property holds.

We now address the issue of the regularity of the control function $V_{\text {hum }}$ obtained by minimizing the functional $J$ in (18). To be more precise, we analyze whether this control preserves the smoothness of the initial data to be controlled.

According to a counterexample that we will present later on in Section 3.3 , we will see that, under the very general assumptions under consideration, no smoothness of the control computed by the minimization of the functional $J$ in $(96$ can be expected.

We thus propose an alternate method, based on HUM, which yields a control of minimal norm in some weighted (in time) $L^{2}$ space, and for which we prove that, with no further assumptions, if $x^{0} \in X_{1}$, then this control function belongs to $H_{0}^{1}(0, T ; \mathscr{U})$. Thus, this result can be readily applied to the most relevant examples, as it is for instance the case of the wave equation with Dirichlet boundary control. In particular, this implies that the controlled solution $x$ of $[13)$ belongs to $C^{1}([0, T], X)$ and also, in various situations (see Section 3.5), to a strict subspace of $X$ for all time $t \in[0, T]$, which will reflect the extra regularity of the initial data to be controlled. In particular, if $B B^{*}$ maps $X_{1}$ into $X_{1}$, then the controlled solution $x$ will belong to $C^{0}\left([0, T] ; X_{1}\right)$.

Fix $T>T^{*}$ and choose $\delta>0$ such that $T-2 \delta \geq T^{*}$. Let $\eta=\eta(t) \in L^{\infty}(\mathbb{R})$ be such that

$$
\eta: \mathbb{R} \rightarrow[0,1], \quad \eta(t)= \begin{cases}0 & \text { if } t \notin(0, T), \\ 1 & \text { if } t \in[\delta, T-\delta] .\end{cases}
$$

In particular, there exists a positive constant $C_{o b s}$ such that any solution $\varphi$ of (14) satisfies

$$
\|\varphi(0)\|_{X}^{2} \leq C_{o b s}^{2} \int_{0}^{T} \eta(t)\left\|B^{*} \varphi(t)\right\|_{\mathscr{U}}^{2} d t .
$$

Then define the functional $J$ by

$$
J\left(\varphi^{T}\right)=\frac{1}{2} \int_{0}^{T} \eta(t)\left\|B^{*} \varphi(t)\right\|_{\mathscr{U}}^{2} d t+\left\langle x^{0}, \varphi(0)\right\rangle_{X},
$$

where $\varphi$ denotes the solution of the adjoint system (14) with initial data $\varphi^{T}$.

Inequality (22) then implies the strict convexity of the functional $J$ and its coercivity, but with respect to the norm

$$
\left\|\varphi^{T}\right\|_{o b s}^{2}=\int_{0}^{T} \eta(t)\left\|B^{*} \varphi(t)\right\|_{\mathscr{U}}^{2} d t
$$

Let us now remark that, since we assumed that $\mathbb{T}$ is a strongly continuous unitary group, the three norms $\left\|\varphi^{T}\right\|_{X},\|\varphi(0)\|_{X}$ and $\left\|\varphi^{T}\right\|_{\text {obs }}$ (in view of (15)-22) are equivalent. 
We are now in position to state our first result:

Proposition 1. Let $x^{0} \in X$. Assume that system (13) is admissible and exactly observable in some time $T^{*}$. Let $T>T^{*}$ and $\eta \in L^{\infty}(\mathbb{R})$ as in 21 .

Then the functional $J$ in (23) has a unique minimizer $\Phi^{T} \in X$ on X. Besides, the function $V$ given by

$$
V(t)=\eta(t) B^{*} \Phi(t)
$$

where $\Phi(t)$ is the solution of (14) with initial datum $\Phi^{T}$, is a control function for system 13. This control can also be characterized as the one of minimal $L^{2}(0, T ; d t / \eta ; \mathscr{U})$-norm among all possible controls for which the solution of 13) satisfies the control requirement (16). Besides,

$$
\int_{0}^{T}\|V(t)\|_{\mathscr{U}}^{2} \frac{d t}{\eta(t)}=\left\|\Phi^{T}\right\|_{o b s}^{2} \leq C_{o b s}^{2}\left\|x_{0}\right\|_{X}^{2}
$$

where $C_{o b s}$ is the constant in the observability inequality (22).

Moreover, this process defines linear maps

$$
\mathbb{V}_{a}:\left\{\begin{array}{l}
X \longrightarrow X^{*}=X \\
x^{0} \mapsto \Phi^{T}
\end{array} \quad \text { and } \quad \mathbb{V}:\left\{\begin{array}{l}
X \longrightarrow L^{2}\left(0, T, \frac{d t}{\eta(t)} ; \mathscr{U}\right) \\
x^{0} \mapsto V .
\end{array}\right.\right.
$$

Besides, $V$ is the unique admissible control function that can be written $v(t)=$ $\eta(t) B^{*} \varphi(t)$ for some $\varphi$ solution of the adjoint equation (14).

This result is similar to those obtained in the context of HUM (see [68] and previous paragraphs) and follows the same lines as Step 1 of the proof of Theorem 1 Normally the weight $\eta$ is simply taken to be $\eta \equiv 1$ on $[0, T]$ while in the present formulation, the fact that it vanishes at $t=0, T$ plays a key role.

The main novelty and advantage of using the weight function $\eta$ is that, with no further assumption on the control operator $B$, the control inherits the regularity of the data to be controlled.

To state our results, it is convenient to introduce, for $s \in \mathbb{R}_{+}$, some notations: $\lceil s\rceil$ denotes the smallest integer satisfying $\lceil s\rceil \geq s,\lfloor s\rfloor$ is the largest integer satisfying $\lfloor s\rfloor \leq s$ and $\{s\}=s-\lfloor s\rfloor$. Finally, the space $C^{s}$ denotes the classical Hölder space.

Theorem $3(\mid[35])$. Assume that the hypotheses of Proposition 1 are satisfied.

Let $s \in \mathbb{R}_{+}$be a nonnegative real number and further assume that $\eta \in C^{\lceil s\rceil}(\mathbb{R})$.

If the initial datum $x^{0}$ to be controlled belongs to $\mathscr{D}\left(A^{s}\right)$, then the minimizer $\Phi^{T}$ given by Proposition 1 and the control function $V$ given by (25), respectively, belong to $\mathscr{D}\left(A^{s}\right)$ and $H_{0}^{s}(0, T ; \mathscr{U})$.

Besides, there exists a positive constant $C_{s}=C_{s}\left(\eta, C_{o b s}, K_{T}\right)$ independent of $x^{0} \in$ $\mathscr{D}\left(A^{s}\right)$ such that

$$
\left\|\Phi^{T}\right\|_{\mathscr{D}\left(A^{s}\right)}^{2}+\|V\|_{H_{0}^{s}(0, T ; \mathscr{U})}^{2} \leq C_{s}\left\|x^{0}\right\|_{\mathscr{D}\left(A^{s}\right)}^{2} .
$$

In other words, the maps $\mathbb{V}_{a}$ and $\mathbb{V}$ defined in 27] satisfy: 


$$
\mathbb{V}_{a}: \mathscr{D}\left(A^{s}\right) \longrightarrow \mathscr{D}\left(A^{s}\right), \quad \mathbb{V}: \mathscr{D}\left(A^{s}\right) \longrightarrow H_{0}^{s}(0, T ; \mathscr{U}) .
$$

In other words, the constructive method we have proposed, strongly inspired by HUM, naturally reads the regularity of the initial data to be controlled, and provides smoother controls for smoother initial data. Note however that if one is interested to the regularity in space of the controlled trajectory, one needs to work slightly more.

Indeed, one of the main consequences of Theorem 3 is the following regularity result for the controlled trajectory:

Corollary 3 ([35]). Under the assumptions of Theorem 3 if the initial datum $x^{0}$ to be controlled belongs to $\mathscr{D}\left(A^{s}\right)$, then the controlled solution $x$ of 13 with the control function $V$ given by Proposition 1 belongs to

$$
C^{s}([0, T] ; X) \underset{k=0}{\lfloor s\rfloor} C^{k}\left([0, T] ; \mathscr{X}_{s-k}\right),
$$

where the spaces $\left(\mathscr{X}_{j}\right)_{j \in \mathbb{N}}$ are defined by induction by

$$
\mathscr{X}_{0}=X, \quad \mathscr{X}_{j}=A^{-1}\left(\mathscr{X}_{j-1}+B B^{*} \mathscr{D}\left(A^{j}\right)\right),
$$

and the spaces $\mathscr{X}_{s}$ for $s \geq 0$ are defined by interpolation by

$$
\mathscr{X}_{s}=\left[\mathscr{X}_{\lfloor s\rfloor}, \mathscr{X}_{[s]}\right]_{\{s\}} \text {. }
$$

The spaces $\mathscr{X}_{j}$ are not explicit in general. However, there are several cases in which they can be shown to be included in Hilbert spaces of the form $\mathscr{D}\left(A^{j}\right)$, which in practical applications to PDE are constituted by functions that are smoother than $X$ with respect to the space variable.

In particular, if $B B^{*}$ maps $\mathscr{D}\left(A^{j}\right)$ to itself for all $j \in \mathbb{N}$, then the spaces $\mathscr{X}_{j}$ can be shown to coincide with $\mathscr{D}\left(A^{j}\right)$ for all $j>0$. Of course, this is sharp, since one cannot expect the controlled solution to be better than $C^{0}\left([0, T] ; \mathscr{D}\left(A^{s}\right)\right)$ for initial data $x^{0} \in \mathscr{D}\left(A^{s}\right)$.

Proof (Sketch of the proof of Theorem 3). We focus on the case $s=1$, the others being completely similar.

Since $V(t)=\eta(t) B^{*} \Phi(t)$ is a control function, for any $\varphi^{T} \in X$, identity 20 holds. Then, assuming that $\varphi^{T}=A^{2} \Phi^{T} \in X$, we get

$$
\int_{0}^{T} \eta(t)\left\langle B^{*} \Phi(t), B^{*} A^{2} \Phi(t)\right\rangle_{\mathscr{U}} d t+\left\langle x^{0}, A^{2} \Phi(0)\right\rangle_{X}=0 .
$$

But 


$$
\begin{aligned}
& \int_{0}^{T} \eta(t)\left\langle B^{*} \Phi(t), B^{*} A^{2} \Phi(t)\right\rangle_{\mathscr{U}} d t \\
& =\int_{0}^{T} \eta(t)\left\langle B^{*} \Phi(t), B^{*} \Phi^{\prime \prime}(t)\right\rangle_{\mathscr{U}} d t \\
& =-\int_{0}^{T} \eta(t)\left\|B^{*} \Phi(t)\right\|_{\mathscr{U}}^{2} d t-\int_{0}^{T} \eta^{\prime}(t)\left\langle B^{*} \Phi(t), B^{*} \Phi^{\prime}(t)\right\rangle_{\mathscr{U}} d t
\end{aligned}
$$

and

$$
\left\langle x^{0}, A^{2} \Phi(0)\right\rangle_{X}=-\left\langle A x^{0}, A \Phi(0)\right\rangle_{X} .
$$

Therefore, assuming some regularity on $\Phi_{T}$, namely $\Phi_{T} \in \mathscr{D}\left(A^{2}\right)$, one can prove

$$
\int_{0}^{T} \eta(t)\left\|B^{*} \Phi^{\prime}(t)\right\|_{U}^{2} d t+\int_{0}^{T} \eta^{\prime}(t)\left\langle B^{*} \Phi(t), B^{*} \Phi^{\prime}(t)\right\rangle_{U} d t+\left\langle A x^{0}, A \Phi(0)\right\rangle_{X}=0 .
$$

But, since $\eta \in C^{1}(\mathbb{R})$, for any $\varepsilon>0$, (the constants $C$ below denote various positive constants which do not depend on $\varepsilon$ and that may change from line to line)

$$
\begin{aligned}
\left|\int_{0}^{T} \eta^{\prime}(t)\left\langle B^{*} \Phi(t), B^{*} \Phi^{\prime}(t)\right\rangle_{\mathscr{U}} d t\right| & \leq \frac{C}{\varepsilon} \int_{0}^{T}\left\|B^{*} \Phi(t)\right\|_{\mathscr{U}}^{2} d t+\varepsilon \int_{0}^{T}\left\|B^{*} \Phi^{\prime}(t)\right\|_{\mathscr{U}}^{2} d t \\
& \leq \frac{C}{\varepsilon}\left\|\Phi^{T}\right\|_{X}^{2}+C \varepsilon\left\|\Phi^{\prime}(T)\right\|_{X}^{2} \\
& \leq \frac{C}{\varepsilon}\left\|\Phi^{T}\right\|_{o b s}^{2}+C \varepsilon\left\|\Phi^{\prime}(0)\right\|_{X}^{2} \\
& \leq \frac{C}{\varepsilon}\left\|\Phi^{T}\right\|_{X}^{2}+C \varepsilon \int_{0}^{T} \eta(t)\left\|B^{*} \Phi^{\prime}(t)\right\|_{\mathscr{U}}^{2} d t,
\end{aligned}
$$

where we used the equivalence of the norms $\left\|\varphi^{T}\right\|_{X},\|\varphi(0)\|_{X},\left\|B^{*} \varphi\right\|_{L^{2}(0, T ; \mathscr{U})}$ and $\left\|\varphi^{T}\right\|_{o b s}$, the admissibility and observability inequalities (15) and (22) and estimate (26).

In particular, taking $\varepsilon>0$ small enough,

$$
\left|\int_{0}^{T} \eta^{\prime}(t)\left\langle B^{*} \Phi(t), B^{*} \Phi^{\prime}(t)\right\rangle_{\mathscr{U}} d t\right| \leq C\left\|x^{0}\right\|_{X}^{2}+\frac{1}{2} \int_{0}^{T} \eta(t)\left\|B^{*} \Phi(t)\right\|_{\mathscr{U}}^{2} d t
$$

It then follows from 33 that

$$
\frac{1}{2} \int_{0}^{T} \eta(t)\left\|B^{*} \Phi^{\prime}(t)\right\|_{\mathscr{U}}^{2} d t \leq C\left\|x^{0}\right\|_{X}^{2}+\left\|A x^{0}\right\|_{X}\left\|A \Phi^{T}\right\|_{X} .
$$

But $\left\|x^{0}\right\|_{X} \leq C\left\|A x^{0}\right\|_{X}$ and, applying the observability inequality 22 to $A \Phi(0)$, which reads

$$
\|A \Phi(0)\|_{X}^{2} \leq C_{o b s}^{2} \int_{0}^{T} \eta(t)\left\|B^{*} \Phi^{\prime}(t)\right\|_{\mathscr{U}}^{2} d t
$$

we obtain 


$$
\left\|A \Phi^{T}\right\|_{X}^{2}=\|A \Phi(0)\|_{X}^{2} \leq C_{o b s}^{2} \int_{0}^{T} \eta(t)\left\|B^{*} \Phi^{\prime}(t)\right\|_{\mathscr{U}}^{2} d t \leq C\left\|A x^{0}\right\|_{X}^{2} .
$$

Since $V^{\prime}=\eta^{\prime} B^{*} \Phi+\eta(t) B^{*} \Phi^{\prime}$,

$$
\int_{0}^{T}\left\|V^{\prime}(t)\right\|_{\mathscr{U}}^{2} d t \leq 2 \int_{0}^{T} \eta^{\prime}(t)^{2}\left\|B^{*} \Phi(t)\right\|_{\mathscr{U}}^{2} d t+2 \int_{0}^{T} \eta(t)^{2}\left\|B^{*} \Phi^{\prime}(t)\right\|_{\mathscr{U}}^{2} d t .
$$

But

$$
\begin{aligned}
\int_{0}^{T} \eta^{\prime}(t)^{2}\left\|B^{*} \Phi(t)\right\|_{\mathscr{U}}^{2} d t & \leq C \int_{0}^{T}\left\|B^{*} \Phi(t)\right\|_{\mathscr{U}}^{2} d t \\
& \leq C\left\|\Phi^{T}\right\|_{X}^{2} \leq C\left\|\Phi^{T}\right\|_{o b s}^{2} \leq C\left\|x^{0}\right\|_{X}^{2},
\end{aligned}
$$

where we used (15), the equivalence of the norms $\left\|\Phi^{T}\right\|_{X}$ and $\|\Phi(0)\|_{X}$, (22) and (26) in the last estimate.

Then estimate (28) follows from estimates (36), (37) and 38).

To make the arguments in the formal proof above rigorous, one should take, instead of $\varphi^{T}=A^{2} \Phi^{T}$, which is a priori not allowed in (7),

$$
\varphi_{\tau}^{T}=\frac{1}{\tau^{2}}\left(\Phi(T+\tau)+\Phi(T-\tau)-2 \Phi^{T}\right)
$$

and then pass to the limit in $\tau \rightarrow 0$.

As we said, the proof for integers $s \in \mathbb{N}$ can be made following the same lines. And the general case $s \geq 0$ can then be deduced using interpolation results. Details can be found in [35].

Remark 6. When the operator $B$ is bounded from $X$ to $\mathscr{U}$, the HUM functional $J$ in (18), without the time cut-off function $\eta$, satisfies the same regularity results as the one in Theorem 3 for $s=1$. For larger $s$, and if one furthermore assumes that $B B^{*} \in \mathfrak{L}\left(\mathscr{D}\left(A^{k}\right)\right)$ for all $k \leq s$, then Theorem 3 holds. One immediately deduces Corollary 3 as well. Of course, in this case, an easy induction argument shows that $\mathscr{X}_{k}=\mathscr{D}\left(A^{k}\right)$ for all $k \leq s$.

The main difference appearing in the proof when $\eta \equiv 1$ is that, when integrating by parts, boundary terms appear at $t=0, T$. But they can be suitably bounded when $B$ is bounded. Note that when the cut-off function $\eta$ is introduced, these boundary terms vanish and are transformed into time-integrated terms that are bounded by the weaker admissibility condition.

Remark 7. Note that such regularity results can be found in [24] for the wave equation with internal control and a control operator satisfying $B B^{*} \in \cap_{k \geq 0} \mathfrak{L}\left(\mathscr{D}\left(A^{k}\right)\right)$. There, the authors propose a thorough study of the operator $\mathbb{V}_{a}$ in 29 and give precise estimates on how it acts on each range of frequencies. This is of course much more precise than the results presented in Theorem 3 .

But the proof of the results in [24] requires the use of very deep technical tools such as microlocal analysis and Littlewood-Paley decomposition. 
Let us also point out the article [64] which illustrates numerically the estimates obtained in [24] on the operator $\mathbb{V}_{a}$ in (27).

Also remark however that our approach, though it yields less precise results in the context of the distributed control wave equation, is much more robust and applies also for boundary control problems and any linear conservative equations.

\section{The Constant Coefficient Wave Equation}

\subsection{Problem Formulation: the 1-d case}

Let us first consider the constant coefficient 1 -d wave equation:

$$
\begin{cases}u_{t t}-u_{x x}=0, & 0<x<1,0<t<T \\ u(0, t)=u(1, t)=0, & 0<t<T \\ u(x, 0)=u^{0}(x), u_{t}(x, 0)=u^{1}(x), & 0<x<1\end{cases}
$$

In (39) $u=u(x, t)$ describes the displacement of a vibrating string occupying $(0,1)$.

The energy of solutions of 39 is conserved in time, i.e.,

$$
E(t)=\frac{1}{2} \int_{0}^{1}\left[\left|u_{x}(x, t)\right|^{2}+\left|u_{t}(x, t)\right|^{2}\right] d x=E(0) \quad \forall 0 \leq t \leq T .
$$

The problem of boundary observability of (39) can be formulated as follows: To give sufficient conditions on $T$ such that there exists $C_{o b s}(T)>0$ for which the following inequality holds for all solutions of (39):

$$
E(0) \leq C_{o b s}(T)^{2} \int_{0}^{T}\left|u_{x}(1, t)\right|^{2} d t .
$$

Inequality 441, when it holds, guarantees that the total energy of solutions can be "observed" from the boundary measurement on the extreme $x=1$. The best constant $C_{o b s}(T)$ in 41 is the so-called observability constant 2

Similarly as in the previous Section, the observability problem above is equivalent to the following boundary controllability property: For any $\left(y^{0}, y^{1}\right) \in L^{2}(0,1) \times$ $H^{-1}(0,1)$ there exists $v \in L^{2}(0, T)$ such that the solution of the controlled wave equation

$$
\begin{cases}y_{t t}-y_{x x}=0, & 0<x<1,0<t<T, \\ y(0, t)=0 ; y(1, t)=v(t), & 0<t<T, \\ y(x, 0)=y^{0}(x), y_{t}(x, 0)=y^{1}(x), & 0<x<1,\end{cases}
$$

${ }^{2}$ Inequality 41 is just an example of a variety of similar observability problems: (a) one could observe the energy concentrated on the extreme $x=0$ or in the two extremes $x=0$ and 1 simultaneously; (b) the $L^{2}(0, T)$-norm of $u_{x}(1, t)$ could be replaced by some other norm; (c) one could also observe the energy concentrated in a subinterval $(\alpha, \beta)$ of $(0,1)$, etc. 
satisfies

$$
y(x, T)=y_{t}(x, T)=0, \quad 0<x<1 .
$$

Note that system (39) fits in the abstract setting given in (14) with

$$
\begin{aligned}
\varphi= & \left(\begin{array}{c}
u \\
u_{t}
\end{array}\right), \quad A=\left(\begin{array}{cc}
0 & I d \\
\partial_{x x} & 0
\end{array}\right), \\
& \text { with } \quad X=H_{0}^{1}(0,1) \times L^{2}(0,1), \quad \mathscr{D}(A)=H^{2} \cap H_{0}^{1}(0,1) \times H_{0}^{1}(0,1) .
\end{aligned}
$$

Hence the corresponding control system should be given by duality as in Section 2 However, in the PDE context, it is classical to identify $L^{2}(0,1)$ with its dual. Of course, once this identification is done, though $X$ is an Hilbert space, its dual $X^{*}$ cannot be identified anymore with itself. That explains why the control system (42) is considered with initial data $L^{2}(0,1) \times H^{-1}(0,1)$, which is a natural candidate for $X^{*}$. But our presentation in the abstract setting in Section 2 can still be done in that case, but that would require the introduction of further notations that may be confusing.

Thus, we directly address this example showing why controllability of 42 is a consequence of (41) by a minimization method which yields the control of minimal $L^{2}(0, T)$-norm, similarly as the one developed in the previous Section.

Given $\left(y^{0}, y^{1}\right) \in L^{2}(0,1) \times H^{-1}(0,1)$, a control $V_{\text {hum }} \in L^{2}(0, T)$ can be computed as

$$
V_{\text {hum }}(t)=U_{x}(1, t),
$$

where $U$ is the solution of 39 ) corresponding to initial data $\left(U^{0}, U^{1}\right) \in H_{0}^{1}(0,1) \times$ $L^{2}(0,1)$ minimizing the functional

$$
J\left(\left(u^{0}, u^{1}\right)\right)=\frac{1}{2} \int_{0}^{T}\left|u_{x}(1, t)\right|^{2} d t+\int_{0}^{1} y^{0} u^{1} d x-\left\langle y^{1}, u^{0}\right\rangle_{H^{-1} \times H_{0}^{1}}
$$

in the space $H_{0}^{1}(0,1) \times L^{2}(0,1)$.

Note that $J$ is convex. The continuity of $J$ in $H_{0}^{1}(0,1) \times L^{2}(0,1)$ is guaranteed by the fact that the solutions of (39) satisfy $u_{x}(1, t) \in L^{2}(0, T)$ (the so-called hidden regularity property, that holds also for the Dirichlet problem for the wave equation in several space dimensions; see [60, 68, 69]). More, precisely, for all $T>0$ there exists a constant $K(T)>0$ such that, for all solution of (39),

$$
\int_{0}^{T}\left[\left|u_{x}(0, t)\right|^{2}+\left|u_{x}(1, t)\right|^{2}\right] d t \leq K(T) E(0)
$$

Thus, to prove the existence of a minimizer for $J$, it is sufficient to prove that it is coercive. This is guaranteed by the observability inequality 41]. Also note that the observability inequality (41) also guarantees the strict convexity of $J$ and then the uniqueness of a minimizer for $J$.

Let us see that the minimum of $J$ provides the control. The functional $J$ is of class $C^{1}$. Consequently, the gradient of $J$ at the minimizer vanishes: 


$$
\begin{aligned}
0=\left\langle D J\left(\left(U^{0}, U^{1}\right)\right),\left(w^{0}, w^{1}\right)\right\rangle=\int_{0}^{T} U_{x}(1, t) w_{x}(1, t) d t & \\
& +\int_{0}^{1} y^{0} w^{1} d x-\left\langle y^{1}, w^{0}\right\rangle_{H^{-1} \times H_{0}^{1}}
\end{aligned}
$$

for all $\left(w^{0}, w^{1}\right) \in H_{0}^{1}(0,1) \times L^{2}(0,1)$, where $w$ stands for the solution of 39] with initial data $\left(w^{0}, w^{1}\right)$. By choosing the control as in 45) this identity yields

$$
\int_{0}^{T} V_{\text {hum }}(t) w_{x}(1, t) d t+\int_{0}^{1} y^{0} w^{1} d x-\left\langle y^{1}, w^{0}\right\rangle_{H^{-1} \times H_{0}^{1}}=0 .
$$

On the other hand, multiplying in (42) by $w$ and integrating by parts, we get

$$
\begin{aligned}
\int_{0}^{T} v(t) w_{x}(1, t) d t+\int_{0}^{1} y^{0} w^{1} d x & -\left\langle y^{1}, w^{0}\right\rangle_{H^{-1} \times H_{0}^{1}} \\
& -\int_{0}^{1} y(T) w_{t}(T) d x+\left\langle y_{t}(T), w(T)\right\rangle_{H^{-1} \times H_{0}^{1}}=0 .
\end{aligned}
$$

Combining these two identities we get $\int_{0}^{1} y(T) w_{t}(T) d x-\left\langle y_{t}(T), w(T)\right\rangle_{H^{-1} \times H_{0}^{1}}=0$ for all $\left(w^{0}, w^{1}\right) \in H_{0}^{1}(0,1) \times L^{2}(0,1)$, which is equivalent to the exact controllability condition (43).

This argument shows that observability implies controllability. The reverse is also true. If controllability holds, then, using Banach closed graph Theorem, the linear map that to all initial data $\left(y^{0}, y^{1}\right) \in L^{2}(0,1) \times H^{-1}(0,1)$ of the state equation (42) associates the control of the minimal $L^{2}(0, T)$-norm, which can be still denoted by $V_{\text {hum }}$ in view of Remark 4, is bounded. Multiplying the state equation (42) with that control by $u$, solution of (39), and using (43), we obtain

$$
\int_{0}^{T} V_{\text {hum }}(t) u_{x}(1, t) d t+\int_{0}^{1} y^{0} u^{1} d x-\left\langle y^{1}, u^{0}\right\rangle_{H^{-1} \times H_{0}^{1}}=0 .
$$

Consequently,

$$
\begin{array}{r}
\left|\int_{0}^{1}\left[y^{0} u^{1}-y^{1} u^{0}\right] d x\right|=\left|\int_{0}^{T} V_{\text {hum }}(t) u_{x}(1, t) d t\right| \leq\|v\|_{L^{2}(0, T)}\left\|u_{x}(1, t)\right\|_{L^{2}(0, T)} \\
\leq C||\left(y^{0}, y^{1}\right)\left\|_{L^{2}(0,1) \times H^{-1}(0,1)}\right\| u_{x}(1, t) \|_{L^{2}(0, T)}
\end{array}
$$

for all $\left(y^{0}, y^{1}\right) \in L^{2}(0,1) \times H^{-1}(0,1)$, which implies the observability inequality (41).

Throughout this paper we shall mainly focus on the problem of observability. However, in view of the equivalence above, all the results we present have immediate consequences for controllability. The most important ones will also be stated. Note, however, that controllability is not the only application of the observability inequalities, which are also of systematic use in the context of inverse problems 
([12, 58, 56, 59]). We shall discuss this issue briefly in open problem \# 6 in Section 7.2

Remark 8. Note that here, we consider the adjoint equation (39) with initial data at time $t=0$, whereas in the previous Section, we have considered the adjoint equation (14) with initial data at time $t=T$. This can be done because of the time-reversibility of the wave equation under consideration.

\subsection{Observability for the 1-d wave equation}

The following holds.

Proposition 2. For any $T \geq 2$, system (39) is observable. In other words, for any $T \geq 2$ there exists $C_{\text {obs }}(T)>0$ such that (41) holds for any solution of 39 . Conversely, if $T<2$, [39] is not observable, or, equivalently,

$$
\inf _{u \text { solution of } 39}\left[\frac{1}{E(0)} \int_{0}^{T}\left|u_{x}(1, t)\right|^{2} d t\right]=0 .
$$

The proof of observability for $T \geq 2$ can be carried out in several ways, including Fourier series (and generalizations to non-harmonic Fourier series, see [105]), multipliers (Komornik [60]; Lions [68, 69]), sidewise energy estimates ([110]), Carleman inequalities (Zhang [106]), and microloca $]^{3}$ tools (Bardos, Lebeau, and Rauch [6]; Burq and Gérard [14]).

Let us explain how it can be proved using Fourier series. Solutions of 39 b can be written in the form

$$
\begin{aligned}
& u(x, t)=\sum_{k \geq 1}\left(a_{k} \cos (k \pi t)+\frac{b_{k}}{k \pi} \sin (k \pi t)\right) \sin (k \pi x), \\
& u^{0}(x)=\sum_{k \geq 1} a_{k} \sin (k \pi x), \quad u^{1}(x)=\sum_{k \geq 1} b_{k} \sin (k \pi x) .
\end{aligned}
$$

It follows that

$$
E(0)=\frac{1}{4} \sum_{k \geq 1}\left[a_{k}^{2} k^{2} \pi^{2}+b_{k}^{2}\right]
$$

On the other hand,

$$
u_{x}(1, t)=\sum_{k \geq 1}(-1)^{k}\left[k \pi a_{k} \sin (k \pi t)+b_{k} \cos (k \pi t)\right] .
$$

\footnotetext{
${ }^{3}$ Microlocal analysis deals, roughly speaking, with the possibility of localizing functions and its singularities not only in the physical space but also in the frequency domain. Localization in the frequency domain may be done according to the size of frequencies but also to sectors in the euclidean space in which they belong to. This allows introducing the notion of microlocal regularity; see, for instance, [48].
} 


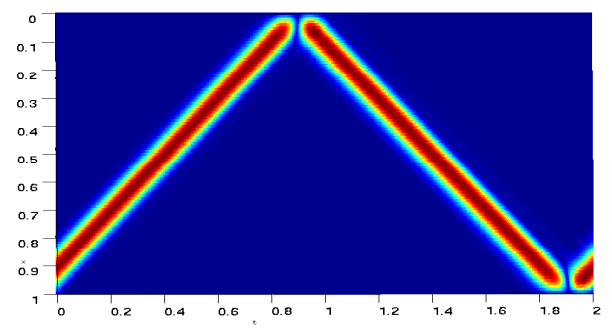

Fig. 1 Wave localized at $t=0$ near the endpoint $x=1$ that propagates with velocity 1 to the left, bounces at $x=0$, and reaches $x=1$ again in a time of the order of 2 .

Using the orthogonality properties of $\sin (k \pi t)$ and $\cos (k \pi t)$ in $L^{2}(0,2)$, it follows that

$$
\int_{0}^{2}\left|u_{x}(1, t)\right|^{2} d t=\sum_{k \geq 1}\left(\pi^{2} k^{2} a_{k}^{2}+b_{k}^{2}\right) .
$$

The two identities above show that the observability inequality holds when $T=2$ and therefore for any $T>2$ as well. In fact, in this particular case, we even have the identity

$$
E(0)=\frac{1}{4} \int_{0}^{2}\left|u_{x}(1, t)\right|^{2} d t .
$$

On the other hand, for $T<2$ the observability inequality does not hold. Indeed, suppose that $T=2-2 \delta$ with $\delta \in(0,2)$. Solve

$$
u_{t t}-u_{x x}=0, \quad(x, t) \in(0,1) \times(0, T), \quad u(0, t)=u(1, t)=0, \quad 0<t<T,
$$

with data at time $t=T / 2=1-\delta$ with support in the subinterval $(0, \delta)$. This solution is such that $u_{x}(1, t)=0$ for $0<t<T=2-2 \delta$ since the segment $x=1, t \in(0, T)$ remains outside the domain of influence of the space segment $t=T / 2, x \in(0, \delta)$ (see Figure 1).

Note that the observability time $(T=2)$ is twice the length of the string. This is due to the fact that an initial disturbance concentrated near $x=1$ may propagate to the left (in the space variable) as $t$ increases and only reach the extreme $x=1$ of the interval after bouncing at the left extreme $x=0$ (as described in Figure 1).

As we have seen, in one dimension and with constant coefficients, the observability inequality is easy to understand. The same results are true for sufficiently smooth coefficients ( $B V$-regularity suffices). However, when the coefficients are simply Hölder continuous, these properties may fail, thereby contradicting an initial intuition (see [19]). 


\subsection{Computing the boundary control}

In this Section, we compute explicitly the control function given by HUM. As a consequence, we will explain in particular that one cannot hope similar regularity results as the ones in Corollary 3 when no cut-off function in time is introduced within the functional $J$ as in 23.

Let us consider the 1-d wave equation (42) controlled by the boundary in time $T=4$. The time $T=4$ is larger than the critical time of controllability, corresponding to $T^{*}=2$, which is the time needed by the waves to go from $x=1$ to $x=0$ and bounce back at $x=0$.

The application of the classical Hilbert Uniqueness Method in this case consists in minimizing the functional $J$ given by (46) to obtain a control $V_{\text {hum }}$ from (45) in terms of the minimizer of $J$.

We now use the fact that, when the control time horizon is $T=4$ (actually it is true for any even integer), the functional $J$ acts diagonally on the Fourier coefficients of the solutions $u$ of (39) and then the minimizer of $J$ can be computed explicitly.

Using (53) for the solutions of (39), one easily checks that

$$
\begin{aligned}
\frac{1}{2} \int_{0}^{T}\left|u_{x}(1, t)\right|^{2} d t & =\frac{1}{2} \int_{0}^{4}\left|\sum_{|k|=1}^{\infty}\left(a_{k} \cos (k \pi t)+\frac{b_{k}}{k \pi} \sin (k \pi t)\right) k \pi(-1)^{k}\right|^{2} d t \\
& =\sum_{k=1}^{\infty}\left(\left|a_{k}\right|^{2} k^{2} \pi^{2}+\left|b_{k}\right|^{2}\right) .
\end{aligned}
$$

The initial datum to be controlled $\left(y^{0}, y^{1}\right) \in L^{2}(0,1) \times H^{-1}(0,1)$ can be written in Fourier series as

$$
\left(y^{0}, y^{1}\right)=\sum_{k=1}^{\infty}\left(\hat{y}_{k}^{0}, \hat{y}_{k}^{1}\right) \sin (k \pi x)
$$

with

$$
\sum_{k=1}^{\infty}\left(\left|\hat{y}_{k}^{0}\right|^{2}+\frac{\left|\hat{y}_{k}^{1}\right|^{2}}{k^{2} \pi^{2}}\right)<\infty .
$$

Thus, for $\left(u^{0}, u^{1}\right)$ as in 53 ,

$$
J\left(\left(u^{0}, u^{1}\right)\right)=\sum_{k=1}^{\infty}\left(\left|a_{k}\right|^{2} k^{2} \pi^{2}+\left|b_{k}\right|^{2}\right)+\frac{1}{2} \sum_{k=1}^{\infty}\left(\hat{y}_{k}^{0} b_{k}-\hat{y}_{k}^{1} a_{k}\right) .
$$

Therefore the minimizer $\left(U^{0}, U^{1}\right)$ of $J$ can be given as

$$
\left(U^{0}, U^{1}\right)=\sum_{k=1}^{\infty}\left(A_{k}, B_{k}\right) \sin (k \pi x), \quad \text { with }\left\{\begin{array}{l}
A_{k}=\frac{\hat{y}_{k}^{1}}{4 k^{2} \pi^{2}}, \\
B_{k}=-\frac{\hat{y}_{k}^{0}}{4},
\end{array}\right.
$$




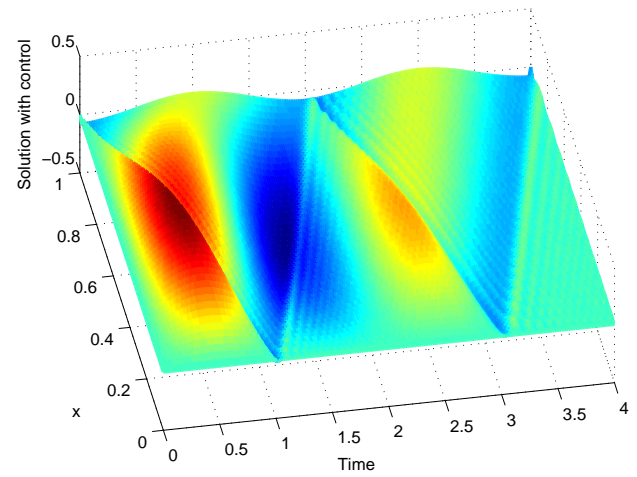

Fig. 2 The controlled trajectory for the wave equation with initial data $\left(y^{0}(x), y^{1}(x)\right)=(0, \sin (\pi x))$ for the HUM control in time $T=4$. A kick is introduced by the control function at $(t, x)=(0,1)$ and travels in the domain, hence making the solution non-smooth.

and the control function $V_{\text {hum }}$ is simply

$$
V_{\text {hum }}(t)=\partial_{x} U(1, t)=\frac{1}{4} \sum_{k=1}^{\infty}(-1)^{k}\left(\frac{\hat{y}_{k}^{1}}{k \pi} \cos (k \pi t)-\hat{y}_{0}^{k} \sin (k \pi t)\right) .
$$

In particular, it is obvious that, for $\left(y^{0}, y^{1}\right) \in H_{0}^{1}(0,1) \times L^{2}(0,1)$, this method yields $\left(U^{0}, U^{1}\right) \in H^{2} \cap H_{0}^{1}(0,1) \times H_{0}^{1}(0,1)$ and $V \in H^{1}(0, T)$.

However,

$$
V_{\text {hum }}(0)=\frac{\sqrt{2}}{4} \sum_{k=1}^{\infty}(-1)^{k} \frac{\hat{y}_{k}^{1}}{k \pi} .
$$

Therefore, if $y^{0} \in H_{0}^{1}(0,1)$, the controlled solution $y$ of 411) with that control function cannot be a strong solution if

$$
\sum_{k=1}^{\infty}(-1)^{k} \frac{\hat{y}_{k}^{1}}{k \pi} \neq 0
$$

whatever the regularity of the initial datum to be controlled is, because the compatibility condition $y(1,0)=V(0)$ does not hold.

Of course, such case happens, for instance when the initial datum to be controlled simply is $\left(y^{0}(x), y^{1}(x)\right)=(0, \sin (\pi x))$. This is illustrated in Figure 2 There, with the control given by HUM, we see that the controlled solution is singular along the characteristic emanating from $(t, x)=(0,1)$.

As this example shows, the regularity of the initial datum does not yield additional regularity for the controlled wave equation when using the HUM control. 


\subsection{The Multidimensional Wave Equation}

In several space dimensions the observability problem for the wave equation is much more complex and cannot be solved using Fourier series except in some particular geometry. The velocity of propagation is still one for all solutions but energy propagates along bicharacteristic rays.

Before going further, let us give the precise definition of bicharacteristic ray. Consider the wave equation with a scalar, positive, and smooth variable coefficient $a=a(x)$ :

$$
u_{t t}-\operatorname{div}(a(x) \nabla u)=0 .
$$

Bicharacteristic rays $s \mapsto(x(s), t(s), \xi(s), \tau(s))$ solve the Hamiltonian system

$$
\begin{cases}x^{\prime}(s)=-a(x) \xi, & t^{\prime}(s)=\tau, \\ \xi^{\prime}(s)=\nabla a(x)|\xi|^{2}, & \tau^{\prime}(s)=0 .\end{cases}
$$

Rays describe the microlocal propagation of energy. The projections of the bicharacteristic rays in the $(x, t)$ variables are the rays of Geometric Optics that play a fundamental role in the analysis of the observation and control properties through the Geometric Control Condition (GCC), that will be introduced below. As time evolves, the rays move in the physical space according to the solutions of (60). Moreover, the direction in the Fourier space $(\xi, \tau)$ in which the energy of solutions is concentrated as they propagate is given precisely by the projection of the bicharacteristic ray in the $(\xi, \tau)$ variables. When the coefficient $a=a(x)$ is constant, the ray is a straight line and carries the energy outward, which is always concentrated in the same direction in the Fourier space, as expected. But for variable coefficients the dynamics is more complex. This Hamiltonian system describes the dynamics of rays in the interior of the domain where the equation is satisfied. When rays reach the boundary they are reflected according to the Snell-Descartes laws of Geometric Optics 4

When the coefficient $a=a(x)$ varies in space, the dynamics of this system may be quite complex and can lead to some unexpected behavior [74].

Let us now address the control problem for smooth domains 5 in the constant coefficient case.

Let $\Omega$ be a bounded domain of $\mathbb{R}^{n}, n \geq 1$, with boundary $\partial \Omega$ of class $C^{2}$, let $\omega$ be an open and nonempty subset of $\Omega$, and let $T>0$. Consider the linear controlled wave equation in the cylinder $Q=\Omega \times(0, T)$ :

$$
\begin{cases}y_{t t}-\Delta y=f 1_{\omega} & \text { in } Q, \\ y=0 & \text { on } \partial \Omega \times(0, T) \\ y(x, 0)=y^{0}(x), y_{t}(x, 0)=y^{1}(x) & \text { in } \Omega .\end{cases}
$$

\footnotetext{
${ }^{4}$ Note, however, that tangent rays may be diffractive or even enter the boundary. We refer to [6] for a deeper discussion of these issues.

${ }^{5}$ We refer to Grisvard [45] for a discussion of these problems in the context of non-smooth domains.
} 


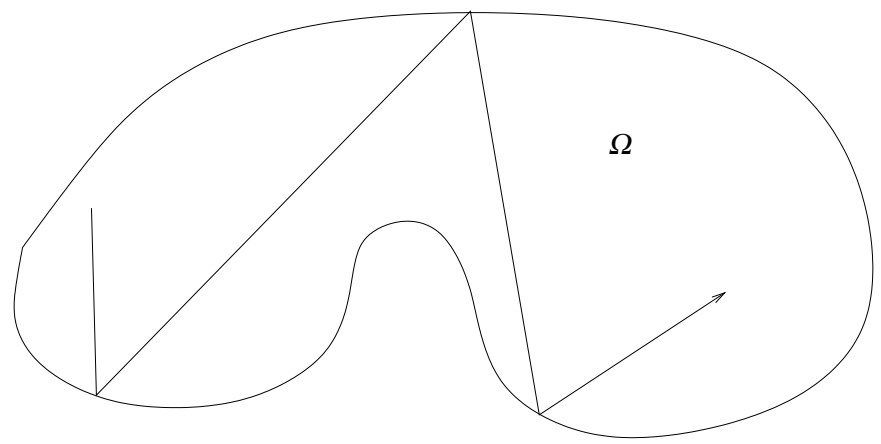

Fig. 3 Ray that propagates inside the domain $\Omega$ following straight lines that are reflected on the boundary according to the laws of Geometric Optics.

In 599 $\Sigma$ represents the lateral boundary of the cylinder $Q$, i.e., $\Sigma=\partial \Omega \times(0, T), 1_{\omega}$ is the characteristic function of the set $\omega, y=y(x, t)$ is the state, and $f=f(x, t)$ is the control variable. Since $f$ is multiplied by $1_{\omega}$ the action of the control is localized in $\omega$.

When $\left(y^{0}, y^{1}\right) \in H_{0}^{1}(\Omega) \times L^{2}(\Omega)$ and $f \in L^{2}(Q)$, the system 59 , has a unique solution $y \in C\left([0, T] ; H_{0}^{1}(\Omega)\right) \cap C^{1}\left([0, T] ; L^{2}(\Omega)\right)$.

The problem of controllability, generally speaking, is as follows: Given $\left(y^{0}, y^{1}\right) \in$ $H_{0}^{1}(\Omega) \times L^{2}(\Omega)$, to find $f \in L^{2}(Q)$ such that the solution of system (59) satisfies

$$
y(T) \equiv y_{t}(T) \equiv 0 .
$$

The method of Section 3 , the so-called HUM, shows that the exact controllability property is equivalent to the following observability inequality:

$$
\left\|\left(u^{0}, u^{1}\right)\right\|_{H_{0}^{1}(\Omega) \times L^{2}(\Omega)}^{2} \leq C \int_{0}^{T} \int_{\omega} u_{t}^{2} d x d t
$$

for every solution of the adjoint uncontrolled system

$$
\begin{cases}u_{t t}-\Delta u=0 & \text { in } \Omega \times(0, T), \\ u=0 & \text { on } \partial \Omega \times(0, T), \\ u(x, 0)=u^{0}(x), u_{t}(x, 0)=u^{1}(x) & \text { in } \Omega .\end{cases}
$$

The main result concerning (63) is that the observability inequality holds if and only if the GCC is satisfied (see, for instance, Bardos, Lebeau, and Rauch [6] and Burq and Gérard [14]): Roughly speaking, the $\operatorname{GCC}$ for $(\Omega, \omega, T)$ states that all rays of Geometric Optics should enter in the domain $\omega$ in a time smaller than $T$.

For instance, when the domain is a ball, the subset of the boundary where the control is being applied needs to contain a point of each diameter. Otherwise, if a diameter skips the control region, it may support solutions that are not observed (see Ralston [87]). In the case of the square domain $\Omega$, observability/controllability fails 
if the control is supported on a set which is strictly smaller than two adjacent sides, as shown in Figure 4

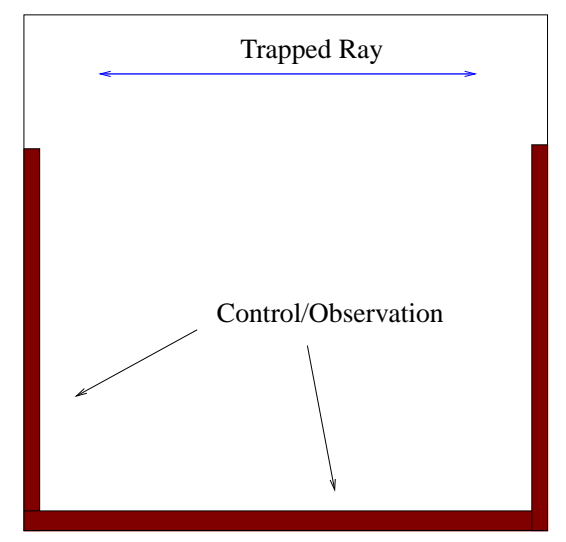

Fig. 4 A geometric configuration in which the GCC is not satisfied, whatever $T>0$ is. The domain where waves evolve is a square. The control is located on a subset of three adjacent sides of the boundary, leaving a small horizontal subsegment uncontrolled. There is a horizontal line that constitutes a ray that bounces back and forth for all time perpendicularly on two points of the vertical boundaries where the control does not act.

Several remarks are in order.

Remark 9. Since we are dealing with solutions of the wave equation, for the GCC to hold, the control time $T$ has to be sufficiently large due to the finite speed of propagation, the trivial case $\omega=\Omega$ being the exception. However, the time being large enough does not suffice, since the control subdomain $\omega$ needs to satisfy the GCC in a finite time. Figure 4 provides an example of this fact.

Remark 10. Most of the literature on the controllability of the wave equation has been written in the framework of the boundary control problem discussed in the previous Section in the 1-dimensional setting. The control problems formulated above for (59) are usually referred to as internal controllability problems since the control acts on the subset $\omega$ of $\Omega$. The latter is easier to deal with since it avoids considering non homogeneous boundary conditions, in which case solutions have to be defined in the sense of transposition [68, 69] and lie in $C^{0}\left([0, T] ; L^{2}(\Omega)\right) \cap$ $C^{1}\left([0, T] ; H^{-1}(\Omega)\right)$ for boundary controls in $L^{2}((0, T) \times \partial \Omega)$.

Note that, if $\Gamma$ denotes an open subset of the boundary $\partial \Omega$, the HUM then expresses the link between controllability of data in $L^{2}(\Omega) \times H^{-1}(\Omega)$ with controls in $L^{2}((0, T) \times \Gamma)$ with the following observability inequality, of course similar to 411): There exists a constant $C_{o b s}$ such that every solution of the adjoint control system (64) satisfies

$$
\left\|\left(u^{0}, u^{1}\right)\right\|_{H_{0}^{1}(\Omega) \times L^{2}(\Omega)}^{2} \leq C_{o b s}^{2} \int_{0}^{T} \int_{\Gamma}\left|\partial_{n} u\right|^{2} d \sigma d t
$$


for every solution of the adjoint uncontrolled system (64).

Let us now discuss what is known about (65):

(a) Using multiplier techniques, Ho [47] proved that if one considers subsets of $\Gamma$ of the form $\Gamma\left(x^{0}\right)=\left\{x \in \Gamma:\left(x-x^{0}\right) \cdot n(x)>0\right\}$ for some $x^{0} \in \mathbb{R}^{n}$ (we denote by $n(x)$ the outward unit normal to $\Omega$ in $x \in \Gamma$ and by $\cdot$ the scalar product in $\mathbb{R}^{n}$ ) and if $T>0$ is large enough, the following boundary observability inequality holds:

$$
\left\|\left(u^{0}, u^{1}\right)\right\|_{H_{0}^{1}(\Omega) \times L^{2}(\Omega)}^{2} \leq C_{o b s}^{2} \int_{0}^{T} \int_{\Gamma\left(x^{0}\right)}\left|\partial_{n} u\right|^{2} d \sigma d t
$$

for all $\left(u^{0}, u^{1}\right) \in H_{0}^{1}(\Omega) \times L^{2}(\Omega)$, which is the observability inequality that is required to solve the boundary controllability problem.

Later, (66) was proved in [68, 69] for any $T>T\left(x^{0}\right)=2\left\|x-x^{0}\right\|_{L^{\infty}(\Omega)}$. This is the optimal observability time that one may derive by means of this multiplier (see Osses [84] for other variants).

Proceeding as in [68], one can easily prove that (66) implies (63) when $\omega$ is a neighborhood of $\Gamma\left(x^{0}\right)$ in $\Omega$, i.e., $\omega=\Omega \cap \Theta$, where $\Theta$ is a neighborhood of $\Gamma\left(x^{0}\right)$ in $\mathbb{R}^{n}$, with $T>2\left\|x-x^{0}\right\|_{L^{\infty}(\Omega \backslash \omega)}$. In particular, exact controllability holds when $\omega$ is a neighborhood of the boundary of $\Omega$.

(b) Bardos, Lebeau, and Rauch [6] proved that, in the class of $C^{\infty}$ domains, the observability inequality 63 ) holds if and only if $(\Omega, \Gamma, T)$ satisfies the GCC: Every ray of Geometric Optics that propagates in $\Omega$ and is reflected on its boundary $\partial \Omega$ intersects $\Gamma$ at a non-diffractive point in time less than $T$.

This result was proved by means of microlocal analysis. Later the microlocal approach was simplified by Burq [7] by using the microlocal defect measures introduced by Gérard [38] in the context of homogenization and kinetic equations. In [7] the GCC was shown to be sufficient for exact controllability for domains $\Omega$ of class $C^{3}$ and equations with $C^{2}$ coefficients. The result for variable coefficients is the same: The observability inequality and, thus, the exact controllability property hold if and only if all rays of Geometric Optics intersect the control region before the control time. However, it is important to note that, although in the constant coefficient equation all rays are straight lines, in the variable coefficient case this is no longer the case, which makes it harder to gain intuition about the GCC.

\subsection{Smoothness properties}

Note that the results in Section 2.3 also apply once observability (63) holds. In particular, adding a cut-off function in time $\eta(t)$ as in 21 within the functional $J$ in (46) implies gentle regularity results for the corresponding minimizers of $J$ and the corresponding control functions. 


\subsubsection{Internal Control Operators}

Assume that, for some time $T^{*}$,

$$
\left\|\left(u^{0}, u^{1}\right)\right\|_{H_{0}^{1}(\Omega) \times L^{2}(\Omega)}^{2} \leq C_{o b s}^{2} \int_{0}^{T^{*}} \int_{\Omega} \chi_{\omega}^{2} u_{t}^{2} d x d t,
$$

for all solutions $u$ of (64), where $\chi_{\omega}=\chi_{\omega}(x)$ is a non-negative function on $\Omega$ which is localized in $\omega$. (21).

Let $T>T^{*}$, choose $\delta>0$ such that $T-2 \delta \geq T^{*}$ and fix a function $\eta$ satisfying

Then the functional $J$ introduced in $(23)$ is defined for $\left(u^{0}, u^{1}\right) \in L^{2}(\Omega) \times$ $H^{-1}(\Omega)$ by

$$
\begin{aligned}
J\left(\left(u^{0}, u^{1}\right)\right)=\frac{1}{2} \int_{0}^{T} \int_{\Omega} \eta(t) \chi_{\omega}^{2}(x)|u(x, t)|^{2} d x d t & +\int_{\Omega} y^{1} u^{0} d x \\
& -\left\langle y^{0}, u^{1}\right\rangle_{H_{0}^{1}(\Omega) \times H^{-1}(\Omega)}
\end{aligned}
$$

where $u$ is the solution of 64 with initial data $\left(u^{0}, u^{1}\right) \in L^{2}(\Omega) \times H^{-1}(\Omega)$.

This functional is not exactly the one corresponding to the abstract presentation above since we did not identify the energy space $H_{0}^{1}(\Omega) \times L^{2}(\Omega)$ with its dual. We have rather shifted by one derivative the regularity of the adjoint solutions under consideration so that their initial data lie in $L^{2}(\Omega) \times H^{-1}(\Omega)$. Note that this functional is more natural when doing PDE because of the classical identification of $L^{2}(\Omega)$ with its dual.

But now, the relevant estimate is, instead of 67 ,

$$
\left\|\left(u^{0}, u^{1}\right)\right\|_{L^{2}(\Omega) \times H^{-1}(\Omega)}^{2} \leq C_{o b s}^{2} \int_{0}^{T^{*}} \int_{\Omega} \chi_{\omega}^{2}|u|^{2} d x d t,
$$

Let us also emphasize that the two estimates 67 and 69) are completely equivalent and can be deduced one from another by differentiating or integrating the solutions of 64 with respect to the time $t$.

To state our results precisely, we define the operator $A$ as in (44). In particular, $\mathscr{D}\left(A^{s}\right)$ is the space $H^{s+1}(\Omega) \times H^{s}(\Omega)$ for $s \geq 0$ with compatibility boundary conditions depending on $s \geq 0$. To be more precise, $\left(y^{0}, y^{1}\right) \in \mathscr{D}\left(A^{s}\right)$ if and only $\left(y^{0}, y^{1}\right) \in H^{s+1}(\Omega) \times H^{s}(\Omega)$ and satisfies

$$
y_{\mid \partial \Omega}^{0}=-\Delta y_{\mid \partial \Omega}^{0}=(-\Delta)^{j} y_{\mid \partial \Omega}^{0}=0 \quad j \in\{0, \cdots,\lfloor s / 2+1 / 4\rfloor\}
$$

and

$$
y_{\mid \partial \Omega}^{1}=-\Delta y_{\mid \partial \Omega}^{1}=(-\Delta)^{j} y_{\mid \partial \Omega}^{1}=0 \quad j \in\{0, \cdots,\lfloor s / 2-1 / 4\rfloor\} .
$$

To simplify the notations in a consistent way, we also introduce $\mathscr{D}\left(A^{s}\right)$ for $s \in$ $[-1,0]$, which is, for $s=-1, \mathscr{D}\left(A^{-1}\right)=L^{2}(\Omega) \times H^{-1}(\Omega)$, for $s=0, \mathscr{D}\left(A^{0}\right)=$ 
$X=H_{0}^{1}(\Omega) \times L^{2}(\Omega)$ and for $s \in(-1,0), \mathscr{D}\left(A^{s}\right)$ is the corresponding interpolation between $\mathscr{D}\left(A^{-1}\right)$ and $X=\mathscr{D}\left(A^{0}\right)$.

Actually, for explaining these notations, we emphasize that we did not identify $X$ with its dual. Therefore, we shall introduce the space $X^{*}=L^{2}(\Omega) \times H^{-1}(\Omega)$, the operator

$$
A^{*}=\left(\begin{array}{cc}
0 & I \\
\Delta & 0
\end{array}\right), \quad \text { with } \mathscr{D}\left(A^{*}\right)=H_{0}^{1}(\Omega) \times L^{2}(\Omega) .
$$

Of course, with the above notations, for all $s \geq 0, \mathscr{D}\left(\left(A^{*}\right)^{s}\right)=\mathscr{D}\left(A^{s-1}\right)$.

Theorem 3 and its corollaries then imply:

Theorem 4. Let $\eta$ be a smooth weight function satisfying (21). Let $\chi_{\omega}$ be a cut-off function as above localizing the support of the control. Then, under the controllability conditions above, given any $\left(y^{0}, y^{1}\right) \in H_{0}^{1}(\Omega) \times L^{2}(\Omega)$, there exists a unique minimizer $\left(U^{0}, U^{1}\right)$ of $J$ over $L^{2}(\Omega) \times H^{-1}(\Omega)$. The function

$$
V(x, t)=\eta(t) \chi_{\omega}(x) U(x, t)
$$

is a control for

$$
\begin{cases}y_{t t}-\Delta y=V \chi_{\omega}, & \text { in } \Omega \times(0, \infty), \\ y=0, & \text { on } \partial \Omega \times(0, \infty) \\ \left(y(0), y_{t}(0)\right)=\left(y^{0}, y^{1}\right) \in H_{0}^{1}(\Omega) \times L^{2}(\Omega)\end{cases}
$$

which is characterized as the control function of minimal $L^{2}\left(0, T ; d t / \eta ; L^{2}(\omega)\right)$ norm, defined by

$$
\|v\|_{L^{2}\left(0, T ; d t / \eta ; L^{2}(\omega)\right)}^{2}=\int_{0}^{T} \int_{\omega}|v(x, t)|^{2} d x \frac{d t}{\eta(t)} .
$$

Furthermore, if the weight function $\eta$ satisfies $\eta \in C^{\infty}(\mathbb{R})$, then if $\left(y^{0}, y^{1}\right)$ belongs to $\mathscr{D}\left(A^{s}\right)$ for some $s \in \mathbb{R}_{+},\left(U_{0}, U_{1}\right) \in \mathscr{D}\left(\left(A^{*}\right)^{s}\right)=\mathscr{D}\left(A^{s-1}\right)$.

In particular, when $\chi_{\omega}$ is smooth and all its normal derivatives vanish at the boundary, the control function $V$ given by (70) belongs to

$$
V \in H^{s}\left(0, T ; L^{2}(\omega)\right) \cap \stackrel{\lfloor s-1\rfloor}{\cap} C_{k=0}^{k}\left([0, T] ; H^{s-k}(\omega)\right),
$$

the controlled solution y of (71) belongs to

$$
\left(y, y_{t}\right) \in C^{s}\left([0, T] ; H_{0}^{1}(\Omega) \times L^{2}(\Omega)\right) \underset{k=0}{\stackrel{\lfloor s\rfloor}{h}} C^{k}\left([0, T] ; \mathscr{D}\left(A^{s-k}\right)\right),
$$

and, in particular,

$$
\left(y, y_{t}\right) \in \underset{k=0}{\stackrel{\lfloor s\rfloor}{\cap}} C^{k}\left([0, T] ; H^{s+1-k}(\Omega) \times H^{s-k}(\Omega)\right) .
$$


Remark that in this case, the time-dependent cut-off function is not needed if $\chi_{\omega}$ is assumed to map $\mathscr{D}\left(A^{s}\right)$ to $\mathscr{D}\left(A^{s}\right)$ for all $s>0$. Note that this requires not only that $\chi_{\omega} \in H^{s}(\Omega)$ but also some suitable compatibility conditions on the boundary, that are satisfied for instance when all the normal derivatives of $\chi_{\omega}$ on the boundary vanish.

For more details, we refer to our work [35].

\subsubsection{Boundary Control Operators}

Let us assume that

$$
\left\|\left(u^{0}, u^{1}\right)\right\|_{H_{0}^{1}(\Omega) \times L^{2}(\Omega)}^{2} \leq C_{o b s}^{2} \int_{0}^{T^{*}} \int_{\partial \Omega} \chi_{\Gamma}^{2}\left|\partial_{n} u\right|^{2} d \sigma d t
$$

for all solutions $u$ of (64), where $\chi_{\Gamma}=\chi_{\Gamma}(x)$ is a function localized on some part $\Gamma$ of the boundary $\partial \Omega$.

Then the functional $J$ introduced in $(23)$ is now defined on $H_{0}^{1}(\Omega) \times L^{2}(\Omega)$ and reads as

$$
\begin{array}{r}
J\left(\left(u^{0}, u^{1}\right)\right)=\frac{1}{2} \int_{0}^{T} \int_{\partial \Omega} \eta(t) \chi_{\Gamma}(x)^{2}\left|\partial_{n} u(x, t)\right|^{2} d \Gamma d t+\int_{0}^{1} y^{0}(x) u^{1}(x, 0) d x \\
-\left\langle y^{1}, u^{0}\right\rangle_{H^{-1}(\Omega) \times H_{0}^{1}(\Omega)},
\end{array}
$$

where $u$ is the solution of 64.

Note that, here again, we have identified $L^{2}(\Omega)$ with its topological dual. This artificially creates a shift between the spaces $X, X^{*}$, and also between $\mathscr{D}\left(A^{j}\right)$ and $\mathscr{D}\left(\left(A^{*}\right)^{j}\right)=\mathscr{D}\left(A^{j+1}\right)$. Besides, this is done in the reverse situation as in the previous paragraph, ie:

- The natural space for the controlled trajectory is $X^{*}=L^{2}(\Omega) \times H^{-1}(\Omega)$ and therefore the controlled trajectory should lie in the space $\mathscr{D}\left(\left(A^{*}\right)^{s}\right)=\mathscr{D}\left(A^{s-1}\right)$.

- The natural space for the adjoint equation is $X=H_{0}^{1}(\Omega) \times L^{2}(\Omega)$ and therefore the regularity of the trajectory of the adjoint equation should be quantified with the spaces $\mathscr{D}\left(A^{s}\right)$.

Then our results imply the following:

Theorem 5. Assume that $\chi_{\Gamma}$ is compactly supported in $\Gamma \subset \partial \Omega$ and that $\eta$ is a smooth weight function satisfying 21]. Also assume (75).

Given any $\left(y^{0}, y^{1}\right) \in L^{2}(\Omega) \times H^{-1}(\Omega)$, there exists a unique minimizer $\left(U^{0}, U^{1}\right)$ of $J$ over $H_{0}^{1}(\Omega) \times L^{2}(\Omega)$. The function

$$
V(x, t)=\eta(t) \chi_{\Gamma}(x) \partial_{n} U(x, t)_{\mid \Gamma}
$$

is a control function for 


$$
\begin{cases}y_{t t}-\Delta y=0, & \text { in } \Omega \times(0, \infty), \\ y=\chi_{\Gamma} v, & \text { on } \partial \Omega \times(0, \infty), \\ \left(y(0), y_{t}(0)\right)=\left(y^{0}, y^{1}\right) & \in H_{0}^{1}(\Omega) \times L^{2}(\Omega),\end{cases}
$$

with target $\left(y(T), y_{t}(T)\right)=(0,0)$.

Besides, $V$ can be characterized as the control function which minimizes the $L^{2}\left(0, T ; d t / \eta ; L^{2}(\Gamma)\right)$-norm, defined by

$$
\|v\|_{L^{2}\left(0, T ; d t / \eta ; L^{2}(\Gamma)\right)}^{2}=\int_{0}^{T} \int_{\Gamma}|v(x, t)|^{2} d \Gamma \frac{d t}{\eta(t)},
$$

among all possible controls.

Furthermore, if the function $\chi$ is smooth, then if $\left(y^{0}, y^{1}\right)$ belongs to $\mathscr{D}\left(\left(A^{*}\right)^{s}\right)=$ $\mathscr{D}\left(A^{s-1}\right)$ for some real number $s \in \mathbb{R}_{+}$, the control function $V$ given by (77) belongs to

$$
V \in H_{0}^{s}\left(0, T ; L^{2}(\Gamma)\right) \underset{k=0}{\stackrel{\lfloor s\rfloor}{\cap}} C^{k}\left([0, T] ; H_{0}^{s-k-1 / 2}(\Gamma)\right)
$$

and $\left(U^{0}, U^{1}\right) \in \mathscr{D}\left(A^{s}\right)$. In particular, the controlled solution y of 78 then belongs to

$$
\left(y, y_{t}\right) \in C^{s}\left([0, T] ; L^{2}(\Omega) \times H^{-1}(\Omega)\right) \underset{k=0}{\lfloor s\rfloor} C^{k}\left([0, T] ; H^{s-k}(\Omega) \times H^{s-1-k}(\Omega)\right) .
$$

\section{1-d Finite Difference Semidiscretizations}

\subsection{Orientation}

In Section 3 we showed how the observability/controllability problem for the constant coefficient wave equation can be solved by Fourier series expansions. We now address the problem of the continuous dependence of the observability constant $C_{o b s}(T)$ in (41) with respect to finite difference space semidiscretizations as the mesh-size parameter $h$ tends to zero. This problem arises naturally in the numerical implementation of the controllability and observability properties of the continuous wave equation but is of independent interest in the analysis of discrete models for vibrations.

There are several important facts and results that deserve emphasis and that we shall discuss below:

- The observability constant for the semidiscrete model tends to infinity for any $T$ as $h \rightarrow 0$. This is related to the fact that the velocity of propagation of solutions tends to zero as $h \rightarrow 0$ and the wavelength of solutions is of the same order as the size of the mesh.

- As a consequence of this fact and of the Banach-Steinhaus theorem, there are initial data for the wave equation for which the controls of the semidiscrete mod- 
els diverge. This proves that one cannot simply rely on the classical convergence (consistency + stability) analysis of the underlying numerical schemes to design algorithms for computing the controls.

However, as we shall explain in Section 5 , one can establish weaker observability results that hold uniformly with respect to $h>0$. As a consequence, see Section 6 , we will be able to propose numerical methods for which "weak" discrete controls converge.

\subsection{Finite Difference Approximations}

Given $N \in \mathbb{N}$ we define $h=1 /(N+1)>0$. We consider the mesh $\left\{x_{j}=j h, j=\right.$ $0, \ldots, N+1\}$ which divides $[0,1]$ into $N+1$ subintervals $I_{j}=\left[x_{j}, x_{j+1}\right], j=0, \ldots, N$.

Consider the following finite difference approximation of the wave equation (39):

$$
\begin{cases}u_{j}^{\prime \prime}-\frac{1}{h^{2}}\left[u_{j+1}+u_{j-1}-2 u_{j}\right]=0, & 0<t<T, j=1, \ldots, N, \\ u_{j}(t)=0, & j=0, N+1,0<t<T, \\ u_{j}(0)=u_{j}^{0}, u_{j}^{\prime}(0)=u_{j}^{1}, & j=1, \ldots, N,\end{cases}
$$

which is a coupled system of $N$ linear differential equations of second order. In 811 the function $u_{j}(t)$ provides an approximation of $u\left(x_{j}, t\right)$ for all $j=1, \ldots, N, u$ being the solution of the continuous wave equation 39). The conditions $u_{0}=u_{N+1}=0$ take account of the homogeneous Dirichlet boundary conditions, and the second order differentiation with respect to $x$ has been replaced by the three-point finite difference. Symbol ' denotes differentiation with respect to the time $t$.

We shall use a vector notation to simplify the expressions. In particular, the column vector

$$
\mathbf{u}_{h}(t)=\left(\begin{array}{c}
u_{1}(t) \\
\vdots \\
u_{N}(t)
\end{array}\right)
$$

will represent the whole set of unknowns of the system. Introducing the matrix

$$
A_{h}=\frac{1}{h^{2}}\left(\begin{array}{cccc}
2 & -1 & 0 & 0 \\
-1 & \ddots & \ddots & 0 \\
0 & \ddots & \ddots & -1 \\
0 & 0 & -1 & 2
\end{array}\right)
$$

the system 81 reads as follows:

$$
\mathbf{u}_{h}^{\prime \prime}(t)+A_{h} \mathbf{u}_{h}(t)=0, \quad 0<t<T ; \quad \mathbf{u}_{h}(0)=\mathbf{u}_{h}^{0}, \mathbf{u}_{h}^{\prime}(0)=\mathbf{u}_{h}^{1} .
$$


The solution $\mathbf{u}_{h}$ of (84) depends also on $h$, but most often we shall denote it simply by $\mathbf{u}$.

The energy of the solutions of 81 is

$$
E_{h}(t)=\frac{h}{2} \sum_{j=0}^{N}\left[\left|u_{j}^{\prime}\right|^{2}+\left|\frac{u_{j+1}-u_{j}}{h}\right|^{2}\right],
$$

and it is constant in time. It is also a natural discretization of the continuous energy (40).

The problem of observability of system 81 can be formulated as follows: To find $T>0$ and $C_{h}(T)>0$ such that

$$
E_{h}(0) \leq C_{h}(T)^{2} \int_{0}^{T}\left|\frac{u_{N}(t)}{h}\right|^{2} d t
$$

holds for all solutions of (81).

Observe that $\left|u_{N} / h\right|^{2}$ is a natural approximation 6 of $\left|u_{x}(1, t)\right|^{2}$ for the solution of the continuous system [39]. Indeed $u_{x}(1, t) \sim\left[u_{N+1}(t)-u_{N}(t)\right] / h$ and, taking into account that $u_{N+1}=0$, it follows that $u_{x}(1, t) \sim-u_{N}(t) / h$.

System (81) is finite-dimensional. Therefore, if observability holds for some $T>$ 0 , then it holds for all $T>0$, as we have seen in Section 3

Note also that the existence of a constant $C_{h}(T)$ in 86 follows from the equivalence of norms in finite dimensional spaces and the fact that if $\mathbf{u}_{h}$ is a solution of (81) that satisfies $u_{N}(t)=u_{N+1}(t)=0$, then $\mathbf{u}_{h}=0$. This can be easily seen on 81) using a iteration argument.

We are interested mainly in the uniformity of the constant $C_{h}(T)$ as $h \rightarrow 0$. If $C_{h}(T)$ remains bounded as $h \rightarrow 0$, we say that system (81) is uniformly observable as $h \rightarrow 0$. Taking into account that the observability of the limit system (39) holds only for $T \geq 2$, it would be natural to expect $T \geq 2$ to be a necessary condition for the uniform observability of 81 . This is indeed the case but, as we shall see, the condition $T \geq 2$ is far from being sufficient. In fact, uniform observability fails for all $T>0$. In order to explain this fact it is convenient to analyze the spectrum of 817 .

Let us consider the eigenvalue problem

$$
-\frac{1}{h^{2}}\left[w_{j+1}+w_{j-1}-2 w_{j}\right]=\lambda w_{j}, \quad j=1, \ldots, N ; \quad w_{0}=w_{N+1}=0 .
$$

The spectrum can be computed explicitly in this case (Isaacson and Keller [55]):

$$
\lambda_{h}^{k}=\frac{4}{h^{2}} \sin ^{2}\left(\frac{k \pi h}{2}\right), \quad k=1, \ldots, N,
$$

and the corresponding eigenvectors are

${ }^{6}$ Here and in what follows $u_{N}$ refers to the $N$ th component of the solution $\mathbf{u}$ of the semidiscrete system, which obviously depends also on $h$. 


$$
\mathbf{w}_{h}^{k}=\left(w_{1, h}^{k}, \ldots, w_{N, h}^{k}\right)^{T}: w_{j, h}^{k}=\sin (k \pi j h), \quad k, j=1, \ldots, N .
$$

Obviously, $\lambda_{h}^{k} \rightarrow \lambda^{k}=k^{2} \pi^{2}$, as $h \rightarrow 0$ for each $k \geq 1, \lambda^{k}=k^{2} \pi^{2}$ being the $k$ th eigenvalue of the continuous wave equation (39). On the other hand we see that the eigenvectors $\mathbf{w}_{h}^{k}$ of the discrete system 87 coincide with the restriction to the mesh points of the eigenfunctions $w^{k}(x)=\sin (k \pi x)$ of the continuous wave equation (39) 7

According to (88) we have $\sqrt{\lambda_{h}^{k}}=\frac{2}{h} \sin \left(\frac{k \pi h}{2}\right)$, and therefore, in a first approximation, we have

$$
\left|\sqrt{\lambda_{h}^{k}}-k \pi\right| \sim \frac{k^{3} \pi^{3} h^{2}}{24} .
$$

This indicates that the spectral convergence is uniform only in the range $k \ll h^{-2 / 3}$, see [91]. Thus, one cannot solve the problem of uniform observability for the semidiscrete system (81) as a consequence of the observability property of the continuous wave equation and a perturbation argument with respect to $h$.

\subsection{Nonuniform Observability}

Multiplying (87) by $j\left(w_{j+1}-w_{j}\right)$, one easily obtains (see [53]) the following identity:

Lemma 1. For any $h>0$ and any eigenvector of 87 associated with the eigenvalue $\lambda$,

$$
h \sum_{j=0}^{N}\left|\frac{w_{j+1}-w_{j}}{h}\right|^{2}=\frac{2}{4-\lambda h^{2}}\left|\frac{w_{N}}{h}\right|^{2} .
$$

We now observe that the largest eigenvalue $\lambda_{h}^{N}$ of 87 is such that $\lambda_{h}^{N} h^{2} \rightarrow$ 4 as $h \rightarrow 0$ and note the following result on nonuniform observability.

Theorem 6. For any $T>0$,

$$
\lim _{h \rightarrow 0} \inf _{\left.\mathbf{u}_{h} \text { solution of } 81\right]}\left[\frac{1}{E_{h}(0)}\left(\int_{0}^{T}\left|\frac{u_{N}}{h}\right|^{2} d t\right)\right]=0 .
$$

Proof (Proof of Theorem 6). We consider solutions of 81) of the form $\mathbf{u}_{h}=$ $\cos \left(\sqrt{\lambda_{h}^{N}} t\right) \mathbf{w}_{h}^{N}$, where $\lambda_{h}^{N}$ and $\mathbf{w}_{h}^{N}$ are the $N$ th eigenvalue and eigenvector of 87, respectively. We have

$$
E_{h}(0)=\frac{h}{2} \sum_{j=0}^{N}\left|\frac{w_{j+1, h}^{N}-w_{j, h}^{N}}{h}\right|^{2}
$$

${ }^{7}$ This is a non generic fact that occurs only for the constant coefficient 1-d problem with uniform meshes. 
and

$$
\int_{0}^{T}\left|\frac{u_{N, h}}{h}\right|^{2} d t=\left|\frac{w_{N, h}^{N}}{h}\right|^{2} \int_{0}^{T} \cos ^{2}\left(\sqrt{\lambda_{h}^{N}} t\right) d t
$$

Taking into account that $\lambda_{h}^{N} \rightarrow \infty$ as $h \rightarrow 0$, it follows that

$$
\int_{0}^{T} \cos ^{2}\left(\sqrt{\lambda_{h}^{N}} t\right) d t \rightarrow T / 2 \quad \text { as } h \rightarrow 0 .
$$

By combining 91, 93, 94 and 95, 92 follows immediately.

It is important to note that the solution we have used in the proof of Theorem 6 is not the only impediment for the uniform observability inequality to hold.

Indeed, let us consider the following solution of the semidiscrete system (81), constituted by the last two eigenvectors:

$$
\mathbf{u}_{h}=\frac{1}{\sqrt{\lambda_{h}^{N}}}\left[\exp \left(i \sqrt{\lambda_{h}^{N}} t\right) \mathbf{w}_{h}^{N}-\frac{w_{N, h}^{n}}{w_{N, h}^{N-1}} \exp \left(i \sqrt{\lambda_{h}^{N-1}} t\right) \mathbf{w}_{h}^{N-1}\right] .
$$

This solution is a simple superposition of two monochromatic semidiscrete waves corresponding to the last two eigenfrequencies of the system. The total energy of this solution is of the order 1 (because each of both components has been normalized in the energy norm and the eigenvectors are orthogonal one to each other). However, the trace of its discrete normal derivative is of the order of $h$ in $L^{2}(0, T)$. This is due to two facts.

- First, the trace of the discrete normal derivative of each eigenvector is very small compared to its total energy.

- Second, and more important, the gap between $\sqrt{\lambda_{h}^{N}}$ and $\sqrt{\lambda_{h}^{N-1}}$ is of the order of $h$, as is shown in Figure 5 left. The wave packet (96) then has a group velocity of the order of $h$.

To be more precise, let us compute $\left|\mathbf{u}_{h}\right|^{2}$, with $\mathbf{u}_{h}$ as in (96):

$$
\begin{array}{r}
\left|u_{j, h}(t)\right|^{2}=\frac{1}{\lambda_{h}^{N}}\left(\left|w_{j, h}^{N}-\frac{w_{N, h}^{n}}{w_{N, h}^{N-1}} w_{j, h}^{N-1}\right|^{2} \cos ^{2}\left(\left(\sqrt{\lambda_{h}^{N}}-\sqrt{\lambda_{h}^{N-1}}\right) \frac{t}{2}\right)\right. \\
\left.+\left|\frac{w_{N, h}^{n}}{w_{N, h}^{N-1}} w_{j, h}^{N-1}+w_{j, h}^{N}\right|^{2} \sin ^{2}\left(\left(\sqrt{\lambda_{h}^{N}}-\sqrt{\lambda_{h}^{N-1}}\right) \frac{t}{2}\right)\right) .
\end{array}
$$

By Taylor expansion, the difference between the two frequencies $\sqrt{\lambda_{h}^{N}}$ and $\sqrt{\lambda_{h}^{N-1}}$ is of the order $h$, and thus we see that the solution is periodic of period of the order of $1 / h$.

Note that here, from 91, explicit computations yield 

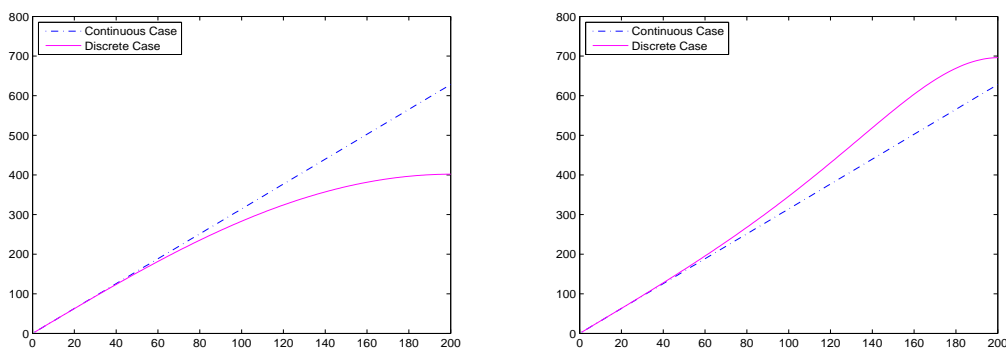

Fig. 5 Left: Square roots of the eigenvalues in the continuous and discrete cases (finite difference semidiscretization). The gaps are clearly independent of $k$ in the continuous case and of order $h$ for large $k$ in the discrete one. Right: Dispersion diagram for the piecewise linear finite element space semidiscretization versus the continuous wave equation.

$$
\begin{aligned}
\left|\frac{u_{N, h}(t)}{h}\right|^{2} & =\left|\frac{w_{N, h}^{N}}{h}\right|^{2} \frac{4}{\lambda_{h}^{N}} \sin ^{2}\left(\left(\sqrt{\lambda_{h}^{N}}-\sqrt{\lambda_{h}^{N-1}}\right) \frac{t}{2}\right) \\
& =\left(1-\frac{\lambda_{h}^{N} h^{2}}{4}\right) \sin ^{2}\left(\left(\sqrt{\lambda_{h}^{N}}-\sqrt{\lambda_{h}^{N-1}}\right) \frac{t}{2}\right) \\
& =\sin ^{2}\left(\frac{\pi h}{2}\right) \sin ^{2}\left(\left(\sqrt{\lambda_{h}^{N}}-\sqrt{\lambda_{h}^{N-1}}\right) \frac{t}{2}\right) .
\end{aligned}
$$

Thus, the integral of the square of the normal derivative of $\mathbf{u}_{h}$ between 0 and $T$ is of order of $h^{4}$, where the smallness comes from both the fact that $\sqrt{\lambda_{h}^{N}}-$ $\sqrt{\lambda_{h}^{N-1}} \simeq h$ and 91 .

High frequency wave packets may be used to show that the observability constant has to blow up at infinite order as $h \rightarrow 0$ (see [75], [76]). To do this it is sufficient to proceed as above but combining an increasing number of eigenfrequencies. Actually, Micu in [77] proved that the constant $C_{h}(T)$ blows up exponentially by means of a careful analysis of the biorthogonal sequences to the family of exponentials $\left\{\exp \left(i \sqrt{\lambda_{h}^{k}} t\right)\right\}_{k=1, \ldots, N}$ as $h \rightarrow 0$.

All these high-frequency pathologies are in fact very closely related to the notion of group velocity. We refer to [104, 101] for an in-depth analysis of this notion that we discuss briefly in the context of this example. Since the eigenvectors $\mathbf{w}_{h}^{k}$ are sinusoidal functions (see (89)) the solutions of the semidiscrete system may be written as linear combinations of complex exponentials (in space-time):

$$
\exp \left( \pm i k \pi\left(\frac{\sqrt{\lambda_{h}^{k}}}{k \pi} t-x\right)\right)
$$


In view of this, we see that each monochromatic wave propagates at a speed

$$
\frac{\sqrt{\lambda_{h}^{k}}}{k \pi}=\frac{2 \sin (k \pi h / 2)}{k \pi h}=\left.\frac{\omega(\xi)}{\xi}\right|_{\{\xi=k \pi h\}}=\left.c(\xi)\right|_{\{\xi=k \pi h\}},
$$

with $\omega(\xi)=2 \sin (\xi / 2)$. This is the so-called phase velocity. The velocity of propagation of monochromatic semidiscrete waves $97 \mathrm{p}$ turns out to be bounded above and below by positive constants, independently of $h$ : $0<2 / \pi \leq c(\xi) \leq 1<\infty$ for all $h>0, \xi \in[0, \pi]$. Note that $[0, \pi]$ is the relevant range of $\xi$. Indeed, $\xi=k \pi h$ and $k=1, \ldots, N, N h=1-h$. This corresponds to frequencies $\zeta=\xi / h$ in $(-\pi / h, \pi / h]$ which is natural due to the sampling of the uniform grid.

But wave packets may travel at a different speed because of the cancellation phenomena we discussed above. The corresponding speed for those semidiscrete wave packets is given by the derivative of $\omega(\cdot)$ (see [101]). At high frequencies $(k \sim N)$ the derivative of $\omega(\xi)$ at $\xi=N \pi h=\pi(1-h)$ is of the order of $h$, the velocity of propagation of the wave packet.

This is illustrated in Figure 6, where we have chosen a discrete initial datum concentrated in space around $x=0.5$ at $t=0$ and in frequency at $\zeta \simeq 0.95 / h$. As one can see, this discrete wave propagate at a very small velocity.

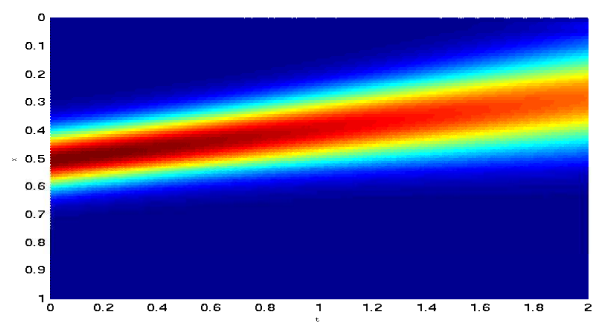

Fig. 6 A discrete wave packet and its propagation. In the horizontal axis we represent the time variable, varying between 0 and 2, and the vertical one the space variable $x$ ranging from 0 to 1 .

The fact that the group velocity is of order $h$ is equivalen 8 to the fact that the gap between $\sqrt{\lambda_{h}^{N-1}}$ and $\sqrt{\lambda_{h}^{N}}$ is of order $h$.

According to this analysis, the group velocity being bounded below is a necessary condition for the uniform observability inequality to hold. Moreover, this is equivalent to a uniform spectral gap condition.

${ }^{8}$ Defining group velocity as the derivative of $\omega$, i.e., of the curve in the dispersion diagram (see Figure 5, is a natural consequence of the classical properties of the superposition of linear harmonic oscillators with close but not identical phases (see [21]). There is a one-to-one correspondence between the group velocity and the spectral gap which may be viewed as a discrete derivative of this diagram. In particular, when the group velocity decreases, the gap between consecutive eigenvalues also decreases. 
The convergence property of the numerical scheme guarantees only that the group velocity of numerical waves is the correct one, close to that of the continuous wave equation, for low-frequency wave packets and this is compatible with the high frequency pathologies mentioned above 9

The careful analysis of this negative example will be useful to design possible remedies, i.e., to propose weaker observability results that would be uniform with respect to the discretization parameter $h>0$. Actually, all the weak observability results that we shall propose in Section 5 (and others, see [36] for extensive references and examples) are based, in a way or another, on removing the high-frequency pathologies generated by the numerical scheme under consideration.

As we will see below in the next paragraph, the fact that the observability inequality (86) is not uniform with respect to $h>0$ has an important consequence in controllability: There are some data to be controlled for which the discrete controls diverge.

Remark 11. According to Figure 5, both finite-difference and finite element methods exhibit a frequency on which the group velocity vanishes. This actually is a generic fact. Indeed, as soon as the discretization method is implemented on a uniform mesh in a symmetric way, the dispersion diagram is given by a continuous function of $\zeta \in(-\pi / h, \pi / h)$ that scales as $\omega(\zeta h) / h$, for some smooth function $\omega$ describing the numerical method under consideration. But this function $\omega$ can actually be defined for $\zeta \in \mathbb{R}$ as the output of the discrete laplacian when the input is $\exp (i \zeta x)$. Doing that, one easily checks that $\omega$ is necessarily $2 \pi$-periodic. According to this, if $\omega$ is smooth, it necessarily has a critical point in $(-\pi, \pi)$.

Therefore, the existence of waves traveling at zero group velocity is generic with respect to the discretization schemes.

To our knowledge, only the mixed finite element method escapes this pathological fact, but this is so since it corresponds to a non-smooth dispersion raltion $\omega(\xi)=2 \tan (\xi / 2)$, which is produced by introducing a mass matrix that degenerates at frequency of order $\pi / h$ where the dispersion relation of the discretization of the laplacian has a critical point. We refer to [17] for a more precise discussion on that particular numerical scheme.

\subsection{Blow up of discrete controls}

This Section is devoted to analyze the consequences of the negative results on observability obtained in Theorem 6 at the level of the controllability of the semidiscrete wave equation (98). The finite-dimensional control system reads as follows

\footnotetext{
${ }^{9}$ Note that in Figure 5 both for finite differences and elements, the semidiscrete and continuous curves are tangent at low frequencies. This is in agreement with the convergence property of the numerical scheme under consideration and with the fact that low-frequency wave packets travel essentially with the velocity of the continuous model.
} 


$$
\begin{cases}y_{j}^{\prime \prime}-\frac{1}{h^{2}}\left[y_{j+1}+y_{j-1}-2 y_{j}\right]=0, & 0<t<T, j=1, \ldots, N, \\ y_{0}(0, t)=0 ; y_{N+1}(1, t)=v(t), & 0<t<T \\ y_{j}(0)=y_{j}^{0}, y_{j}^{\prime}(0)=y_{j}^{1}, & j=1, \ldots, N,\end{cases}
$$

and it is the semidiscrete version of the controlled wave equation (42).

It is easy to see that this semidiscrete system, for all $h>0$ and all $T>0$, is exactly controllable because the Kalman rank condition is satisfied. More precisely, for any given $T>0, h>0$ and initial data $\left(\mathbf{y}_{h}^{0}, \mathbf{y}_{h}^{1}\right)$, there exists a control $\mathbf{v}_{h} \in L^{2}(0, T)$ such that

$$
\mathbf{y}_{h}(T)=\mathbf{y}_{h}^{\prime}(T)=0 .
$$

But, of course, we are interested in the limit process $h \rightarrow 0$. In particular, we would like to understand whether, when the initial data in (98) are "fixed" 10 to be $\left(y^{0}, y^{1}\right) \in L^{2}(0,1) \times H^{-1}(0,1)$, the controls $\mathbf{v}_{h}$ of (98) converge in $L^{2}(0, T)$ as $h \rightarrow 0$ to the control of the continuous wave equation (42). The negative results on the observability problem, and the fact that these two problems, observability and controllability are equivalent, see Section 2 make us predict that, in fact, the convergence of the controls may fail. This is what happens in practice, indeed. In fact for suitable choices of the initial data the controls may diverge as $h \rightarrow 0$, whatever $T>0$ is.

This negative result shows that the discrete approach to numerical control may fail. In other words, controlling a numerical approximation of a controllable system is not necessarily a good way of computing an approximation of the control of the PDE model. Summarizing, the stability and convergence of the numerical scheme for solving the initial-boundary value problem do not guarantee its stability at the level of controllability.

\subsubsection{Controllability of the discrete schemes}

In this Section, we prove that the discrete systems (98) are exactly controllable for any $h>0$ and characterize the controls of minimal norm. This actually is a byproduct of (86) and Section 2.1 We only rewrite it in our setting for the convenience of the reader.

Theorem 7. For any $T>0$ and $h>0$ system (98) is exactly controllable. More precisely, for any $\left(\mathbf{y}_{h}^{0}, \mathbf{y}_{h}^{1}\right) \in \mathbb{R}^{N} \times \mathbb{R}^{N}$, there exists a control $\mathbf{V}_{\text {hum }, h} \in L^{2}(0, T)$ given by HUM such that the solution of (98) satisfies (99).

Moreover, the control $\mathbf{V}_{\text {hum, }}$ of minimal $L^{2}(0, T)$-norm can be characterized through the minimization of the functional

$$
J_{h}\left(\left(\mathbf{u}_{h}^{0}, \mathbf{u}_{h}^{1}\right)\right)=\frac{1}{2} \int_{0}^{T}\left|\frac{u_{N}(t)}{h}\right|^{2} d t+h \sum_{j=1}^{N} y_{j}^{0} u_{j}^{1}-h \sum_{j=1}^{N} y_{j}^{1} u_{j}^{0},
$$

\footnotetext{
${ }^{10}$ For given initial data $\left(y^{0}, y^{1}\right)$, the initial data for the controlled semidiscrete system 98 are taken to be approximations of $\left(y^{0}, y^{1}\right)$ on the discrete mesh. The convergence of the controls $\mathbf{v}_{h}$ in $L^{2}(0, T)$ is then analyzed for the controls corresponding to these approximate initial data.
} 
in $\mathbb{R}^{N} \times \mathbb{R}^{N}$, where $\mathbf{u}_{h}$ is the solution of the adjoint system 81 . More precisely, the control $\mathbf{V}_{\text {hum, } h}$ is of the form

$$
\mathbf{V}_{\text {hum }, h}(t)=-\frac{U_{N}(t)}{h},
$$

where $\mathbf{U}_{h}$ is the solution of the adjoint system (81) corresponding to the initial data $\left(\mathbf{U}_{h}^{0}, \mathbf{U}_{h}^{1}\right)$ minimizing the functional $J_{h}$.

For each $h>0$, as explained in Corollary 2 the control function $\mathbf{V}_{\text {hum }, h}$ of minimal $L^{2}(0, T)$-norm of system (98) is given by a linear map $\mathbb{V}_{h}$ of the initial data $\left(\mathbf{y}_{h}^{0}, \mathbf{y}_{h}^{1}\right)$ and can be written as $\mathbf{V}_{\text {hum }, h}=\mathbb{V}_{h}\left(\mathbf{y}_{h}^{0}, \mathbf{y}_{h}^{1}\right)$.

For convenience, for $h>0$ we introduce the norms

$$
\left\|\left(\mathbf{u}_{h}^{0}, \mathbf{u}_{h}^{1}\right)\right\|_{H_{h}^{1} \times L_{h}^{2}}^{2}=h \sum_{j=0}^{N}\left[\left(\frac{u_{j+1}^{0}-u_{j}^{0}}{h}\right)^{2}+\left|u_{j}^{1}\right|^{2}\right]
$$

and

$$
\left\|\left(\mathbf{y}_{h}^{0}, \mathbf{y}_{h}^{1}\right)\right\|_{L_{h}^{2} \times H_{h}^{-1}}=\sup _{\left\|\left(\mathbf{u}_{h}^{0}, \mathbf{u}_{h}^{1}\right)\right\|_{H_{h}^{1} \times L_{h}^{2}}}\left\{h \sum_{j=1}^{N} y_{j}^{0} u_{j}^{1}-h \sum_{j=1}^{N} u_{j}^{0} y_{j}^{1}\right\} .
$$

The first one corresponds to the energy of 855 and the second one stands for the norm of the space in which the solutions of the controlled semidiscrete system belong to.

In particular, if one extends the discrete functions $\left(\mathbf{u}_{h}^{0}, \mathbf{u}_{h}^{1}\right)$ to continuous ones using Fourier extension, denoted by $\left(u_{h}^{0}, u_{h}^{1}\right)$, the following norms are equivalent:

$$
\left\|\left(\mathbf{u}_{h}^{0}, \mathbf{u}_{h}^{1}\right)\right\|_{H_{h}^{1} \times L_{h}^{2}}^{2} \simeq\left\|\left(u_{h}^{0}, u_{h}^{1}\right)\right\|_{H_{0}^{1} \times L^{2}}^{2} .
$$

We thus deduce by duality the equivalence between the norms

$$
\left\|\left(\mathbf{y}_{h}^{0}, \mathbf{y}_{h}^{1}\right)\right\|_{L_{h}^{2} \times H_{h}^{-1}} \simeq\left\|\left(y_{h}^{0}, y_{h}^{1}\right)\right\|_{L^{2} \times H^{-1}}
$$

As a simple consequence of the equivalence stated in Theorem 2, we have

$$
\left\|\mathbb{V}_{h}\right\|_{\mathfrak{L}\left(L_{h}^{2} \times H_{h}^{-1}, L^{2}(0, T)\right)}=\sqrt{2} C_{h}(T),
$$

where $C_{h}(T)$ is the observability constant in (86).

By Theorems 2 and 6 this indicates that the norms of the discrete control operators blow up when $h \rightarrow 0$ :

Proposition 3. We have

$$
\lim _{h \rightarrow 0}\left\|\mathbb{V}_{h}\right\|_{\mathfrak{L}\left(L_{h}^{2} \times H_{h}^{-1}, L^{2}(0, T)\right)}=+\infty .
$$


Remark 12. Identity (104) indicates that the norm of the discrete controls map blows up when $h \rightarrow 0$ at the same rate as $C_{h}(T)$. In view of the results presented in [77], it blows up with an exponential rate.

As a consequence of Proposition 3 there are continuous data $\left(y^{0}, y^{1}\right) \in L^{2}(0,1) \times$ $H^{-1}(0,1)$ for which the sequence of discrete controls computed on the discrete controlled system 98 is not even bounded.

To state our results precisely, we must explain how the continuous data $\left(y^{0}, y^{1}\right)$ are approximated by discrete ones $\left(\mathbf{y}_{h}^{0}, \mathbf{y}_{h}^{1}\right)$.

For $\left(y^{0}, y^{1}\right) \in L^{2}(0,1) \times H^{-1}(0,1)$, with Fourier expansion

$$
\left(y^{0}, y^{1}\right)=\sum_{k=1}^{\infty}\left(\hat{y}_{k}^{0}, \hat{y}_{k}^{1}\right) w^{k},
$$

we introduce a sequence $\left(\mathbb{A}_{h}\right)_{h>0}$ of discretization operators

$$
\begin{aligned}
L^{2}(0,1) \times H^{-1}(0,1) & \rightarrow \mathbb{R}^{N} \times \mathbb{R}^{N}, \\
\mathbb{A}_{h}: \quad\left(y^{0}, y^{1}\right) & \mapsto\left(\mathbf{y}_{h}^{0}, \mathbf{y}_{h}^{1}\right)=\mathbb{A}_{h}\left(y^{0}, y^{1}\right)=\sum_{k=1}^{N}\left(\hat{y}_{k}^{0}, \hat{y}_{k}^{1}\right) \mathbf{w}^{k} .
\end{aligned}
$$

To simplify notations, we will denote similarly by $\mathbb{A}_{h}\left(y^{0}, y^{1}\right)$ the discrete functions and their continuous corresponding Fourier extensions.

These operators $\mathbb{A}_{h}$ map continuous data $\left(y^{0}, y^{1}\right)$ to discrete ones by truncating the Fourier expansion, and describe a natural relevant discretization process for initial data in $L^{2}(0,1) \times H^{-1}(0,1)$.

For instance, as one can easily check, for any $\left(y^{0}, y^{1}\right) \in L^{2}(0,1) \times H^{-1}(0,1)$,

$$
\mathbb{A}_{h}\left(y^{0}, y^{1}\right) \underset{h \rightarrow 0}{\longrightarrow}\left(y^{0}, y^{1}\right) \quad \text { in } L^{2}(0,1) \times H^{-1}(0,1) .
$$

We now prove the following divergence result:

Theorem 8. There exists an initial datum $\left(y^{0}, y^{1}\right) \in L^{2}(0,1) \times H^{-1}(0,1)$ such that the sequence $\left(\mathbb{V}_{h} \circ \mathbb{A}_{h}\left(y^{0}, y^{1}\right)\right)_{h>0}$ is not bounded in $L^{2}(0, T)$.

Proof. The proof is by contradiction.

Assume that for all $\left(y^{0}, y^{1}\right) \in L^{2}(0,1) \times H^{-1}(0,1)$, the sequence of discrete controls $\left(\mathbb{V}_{h} \circ \mathbb{A}_{h}\left(y^{0}, y^{1}\right)\right)_{h>0}$ is bounded in $L^{2}(0, T)$.

Then, applying Banach-Steinhaus Theorem (or the Principle of Uniform Boundedness) to the operators $\left(\mathbb{V}_{h} \circ \mathbb{A}_{h}\right)_{h>0}$, there is a constant $C>0$ such that for all $h>0$ and $\left(y^{0}, y^{1}\right) \in L^{2}(0,1) \times H^{-1}(0,1)$,

$$
\left\|\mathbb{V}_{h} \circ \mathbb{A}_{h}\left(y^{0}, y^{1}\right)\right\|_{L^{2}(0, T)} \leq C\left\|\left(y^{0}, y^{1}\right)\right\|_{L^{2} \times H^{-1}} .
$$

Due to the particular form of $\mathbb{A}_{h}$, this implies that for all

$$
\left(\mathbf{y}_{h}^{0}, \mathbf{y}_{h}^{1}\right)=\sum_{k=1}^{N}\left(\hat{y}_{k, h}^{0}, \hat{y}_{k, h}^{1}\right) \mathbf{w}_{h}^{k}
$$


we have

$$
\left\|\mathbb{V}_{h}\left(\mathbf{y}_{h}^{0}, \mathbf{y}_{h}^{1}\right)\right\|_{L^{2}(0, T)} \leq C\left\|\left(\mathbf{y}_{h}^{0}, \mathbf{y}_{h}^{1}\right)\right\|_{L_{h}^{2} \times H_{h}^{-1}} .
$$

But this is in contradiction with Proposition 3 and the equivalence (103), which proves the result.

Remark 13. According to Theorem 8 , not only the global cost of controllability diverges, but there exist specific initial data such that its cost diverges. This is a direct consequence of the Principle of Uniform Boundedness. As we indicated above here we refer to the cost of controlling the sequence of discrete initial data $\left(\mathbf{y}_{h}^{0}, \mathbf{y}_{h}^{1}\right)$ built specifically from the initial data $\left(y^{0}, y^{1}\right)$ by truncating Fourier series.

But the approximation $\mathbb{A}_{h}$ of the initial data can be defined differently as well, and the result will remain true. For instance, we may take discrete averages of the continuous data over intervals centered on the mesh-points $x_{j}=j h$. Of course, in what concerns $y^{1}$, we have to be particularly careful since the fact that it belongs to $H^{-1}(0,1)$ allows only doing averages against test functions in $H_{0}^{1}(0,1)$. The use of these test functions can be avoided by first, taking a smooth approximation of $y^{1}$ in $H^{-1}(0,1)$ and then taking averages.

Remark 14. This lack of convergence of the semidiscrete controls $\mathbf{V}_{\text {hum }, h}$ towards the continuous one $V$ can be understood easily. Indeed, as we have shown above, the semidiscrete system, even in the absence of controls, generates a lot of spurious high frequency oscillations. The control $\mathbf{V}_{h u m, h}$ of the semidiscrete system (98) has to take all these spurious components into account. When doing this it gets further and further away from the true control $V$ of the continuous wave equation 427, as the numerical experiments in the following Section illustrate.

\subsection{Numerical experiments}

In this Section, we describe some numerical experiments showing both the instability of the numerical controls for suitable initial data to be controlled. These simulations were performed by Alejandro Maass Jr. using Matlab.

We consider the wave equation in time $T=4$ on the space interval $(0,1)$. This suffices for the boundary control of the continuous wave equation for which the minimal time is $T=2$, see Proposition 2

Given an initial datum to be controlled, we can then compute explicitly the control of the continuous equation.

The control function can then be computed explicitly using Fourier series, see Section 3.3 In Figure 8 we present its plot.

The control can also be computed explicitly by using D'Alembert formula. This also explains the form of the control in Figure 7 right, which looks very much like the superposition of the initial data to be controlled.

We now consider the finite-difference semidiscrete approximation of the wave equation by finite-differences. We then compute the exact control of the semidiscrete system (98) for several values of $N$. 

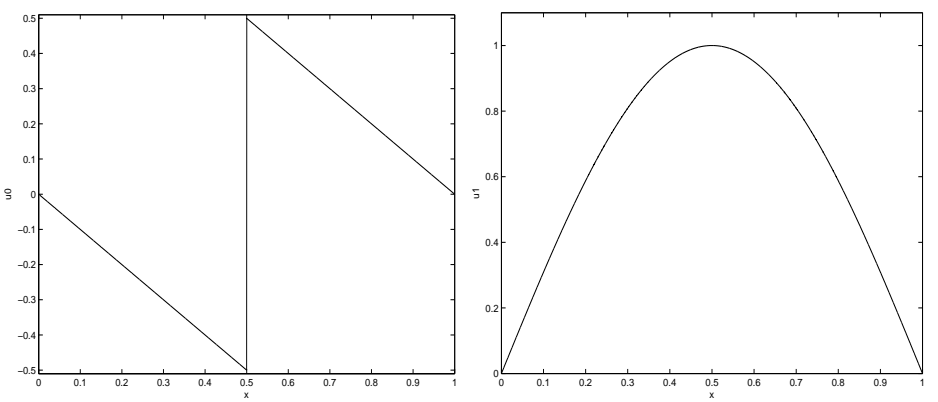

Fig. 7 Plot of the initial datum to be controlled: Left, the position $y^{0}$. Right, the velocity $y^{1}$.

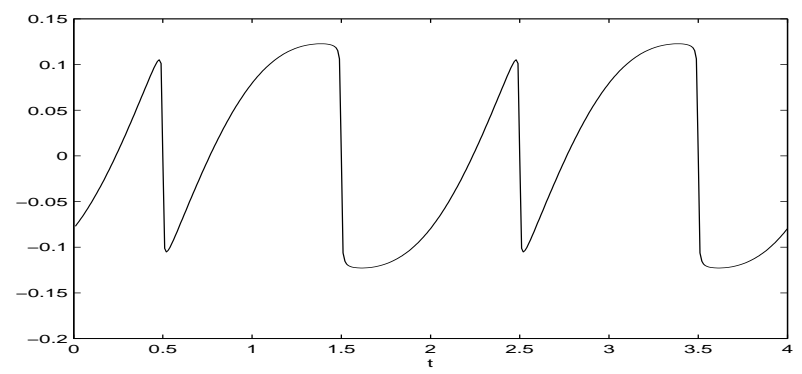

Fig. 8 Plot of the continuous control corresponding to the initial data $\left(y^{0}, y^{1}\right)$ in Figure 7

Of course, in practice, we do not deal with the space semidiscrete adjoint equations (81) but rather with fully discrete approximations. In our experiments we employ the centered discretization in time with time-step $\Delta t=0.5 \mathrm{~h}$, which, of course, guarantees the convergence of the scheme.

Following the discrete approach for numerical control, we compute the controls for the resulting fully discrete system. This is done minimizing the corresponding time-discrete version of the functional $J_{h}$ in (100) using a conjugate gradient algorithm. It turns out that the number of iterations needed for convergence is huge. We stop the conjugate gradient algorithm after 500 iterations. The obtained results are plot in Figure 9 for $N=50$ and $N=150$. Increasing the number of iterations would not change significantly the shape of the obtained controls. Note that they are very far form the shape of the actual control above. This is a clear evidence of the divergence of the discrete procedure to compute an effective numerical approximation of the control by controlling the approximate discrete dynamics. This is due to the very weak observability of the corresponding discrete system which makes the coercivity of the corresponding $J_{h}$ functional to be very weak. This produces two effects. First, the descent algorithms are very slow and, second, the norm of the minimizers is huge. This is what we see in these numerical experiments. 
It is also very surprising that the conjugate gradient method needs so many iterations whereas it minimizes a functional on a finite-dimensional space of dimension $2 N$. Indeed, it is well-known that the conjugate gradient algorithm yields the exact minimizer after $K$ iterations, where $K$ is the size of dimension of the space we are working in, hence, in our case $K=2 N$. Then the functional is very ill-conditioned and the numerical errors cannot be negligible and prevent the conjugate gradient algorithm from converging in $2 \mathrm{~N}$ iterations.

The descent iterative method does converge in 500 iterations when the number of mesh points is less than $N \leq 44$. But the controls one obtains when doing that are very similar to those plotted in Figure 9
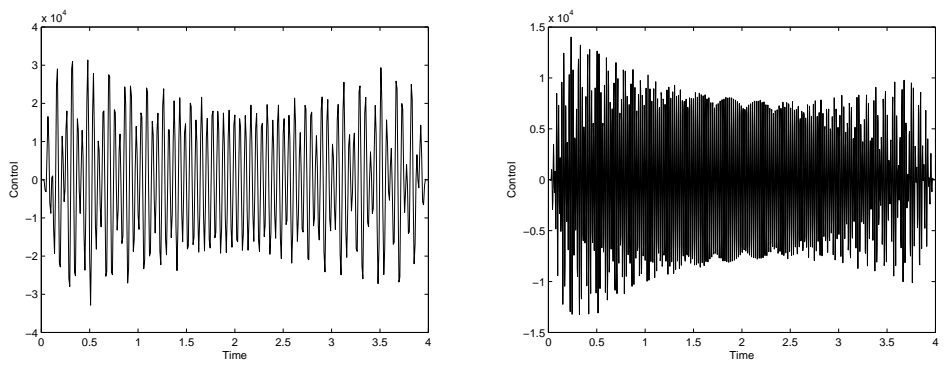

Fig. 9 Divergent evolution of the discrete exact controls when the number $N$ of mesh-points increases. Left: the number of mesh points is $N=50$. Right: $N=150$. In both cases, we plot the control obtained after 500 iterations of the conjugate gradient algorithm for the minimization of $J_{h}$

\section{Remedies for High-Frequency Pathologies}

In the previous Section we have shown that the discrete wave equations are not uniformly (with respect to the space mesh size $h$ ) observable, whatever the time $T>0$ is.

We have mentioned that this is due to high-frequency spurious waves. In this Section, we show that, when employing convenient filtering mechanisms, ruling out the high frequency components, one can recover uniform observability inequalities. At this point it is important to observe that the high-frequency pathologies cannot be avoided by simply taking, for instance, a different approximation of the discrete normal derivative since the fact that the group velocity vanishes is due to the numerical approximation scheme itself and, therefore, cannot be compensated by suitable boundary measurements. One has really to take care of the spurious high frequency solutions that the numerical scheme generates. 


\subsection{Fourier Filtering}

We introduce a Fourier filtering mechanism that consists in eliminating the high frequency Fourier components and restricting the semidiscrete wave equation under consideration to the subspace of solutions generated by the Fourier components corresponding to the eigenvalues $\lambda \leq \gamma h^{-2}$ with $0<\gamma<4$ or with indices $0<j<$ $\delta h^{-1}$ with $0<\delta<1$. In this subspace the observability inequality becomes uniform. Note that these classes of solutions correspond to taking projections of the complete solutions by cutting off all frequencies with $\sqrt{\gamma} h^{-1}<\zeta<2 h^{-1}$.

The following classical result due to Ingham in the theory of nonharmonic Fourier series (see Ingham [54] and Young [105]) is useful for proving the uniform observability of filtered solutions.

Theorem 9 (Ingham [56]). Let $\left\{\mu_{k}\right\}_{k \in \mathbb{Z}}$ be a sequence of real numbers such that $\mu_{k+1}-\mu_{k} \geq \sigma>0$ for all $k \in \mathbb{Z}$. Then for any $T>2 \pi / \sigma$ there exists a positive constant $C(T, \sigma)>0$ depending only on $T$ and $\sigma$ such that

$$
\frac{1}{C(T, \sigma)^{2}} \sum_{k \in \mathbb{Z}}\left|a_{k}\right|^{2} \leq \int_{0}^{T}\left|\sum_{k \in \mathbb{Z}} a_{k} e^{i \mu_{k} t}\right|^{2} d t \leq C(T, \sigma)^{2} \sum_{k \in \mathbb{Z}}\left|a_{k}\right|^{2}
$$

for all sequences of complex numbers $\left\{a_{k}\right\} \in \ell^{2}$.

Remark 15. Ingham's inequality can be viewed as a generalization of the orthogonality property of trigonometric functions we used to prove the observability of the $1-\mathrm{d}$ wave equation in Section 3 , known as Paserval's identity.

Ingham's inequality allows showing that, as soon as the gap condition is satisfied, there is uniform observability provided the time is large enough.

All these facts confirm that a suitable cutoff or filtering of the spurious numerical high frequencies may be a cure for these pathologies.

Let us now describe the basic Fourier filtering mechanism in more detail. We recall that solutions of 81 can be developed in Fourier series as follows:

$$
\mathbf{u}_{h}(t)=\sum_{k=1}^{N}\left(a_{k} \cos \left(\sqrt{\lambda_{h}^{k}} t\right)+\frac{b_{k}}{\sqrt{\lambda_{h}^{k}}} \sin \left(\sqrt{\lambda_{h}^{k}} t\right)\right) \mathbf{w}_{h}^{k},
$$

where $a_{k}, b_{k}$ are the Fourier coefficients of the initial data, i.e., $\mathbf{u}_{h}^{0}=\sum_{k=1}^{N} a_{k} \mathbf{w}_{h}^{k}$, $\mathbf{u}_{h}^{1}=\sum_{k=1}^{N} b_{k} \mathbf{w}_{h}^{k}$.

Given $s>0$, we introduce the following classes of solutions of 81 :

$$
\mathscr{C}_{h}(s)=\left\{\mathbf{u}_{h}(t)=\sum_{\lambda_{h}^{k} \leq s}\left(a_{k} \cos \left(\sqrt{\lambda_{h}^{k} t}\right)+\frac{b_{k}}{\sqrt{\lambda_{h}^{k}}} \sin \left(\sqrt{\lambda_{h}^{k}} t\right)\right) \mathbf{w}_{h}^{k}\right\},
$$


in which the high frequencies corresponding to the indices $j>\lfloor\delta(N+1)\rfloor$ have been cut off. As a consequence of Ingham's inequality and the analysis of the gap of the spectra of the semidiscrete systems we have the following result 11

Theorem 10 (see [53]). For any $\gamma \in(0,4)$ there exists $T(\gamma)>0$ such that for all $T>T(\gamma)$ there exists $C=C(T, \gamma)>0$ such that

$$
\frac{1}{C^{2}} E_{h}(0) \leq \int_{0}^{T}\left|\frac{u_{N}(t)}{h}\right|^{2} d t \leq C^{2} E_{h}(0)
$$

for every solution $\mathbf{u}_{h}$ of 810 in the class $\mathscr{C}_{h}\left(\gamma / h^{2}\right)$ and for all $h>0$. Moreover, the minimal time $T(\gamma)$ for which 109 holds is such that $T(\gamma) \rightarrow 2$ as $\gamma \rightarrow 0$ and $T(\gamma) \rightarrow \infty$ as $\gamma \rightarrow 4$.

Remark 16. Theorem[10guarantees the uniform observability in each class $\mathscr{C}_{h}\left(\gamma / h^{2}\right)$ for all $0<\gamma<4$, provided the time $T$ is larger than $T(\gamma)$.

The last statement in the theorem shows that when the filtering parameter $\gamma$ tends to zero, i.e., when the solutions under consideration contain fewer and fewer frequencies, the time for uniform observability converges to $T=2$, which is the corresponding one for the continuous equation. This is in agreement with the observation that the group velocity of the low-frequency semidiscrete waves coincides with the velocity of propagation in the continuous model.

By contrast, when the filtering parameter increases, i.e., when the solutions under consideration contain more and more frequencies, the time of uniform control tends to infinity. This is in agreement and explains further the negative result showing that, in the absence of filtering, there is no finite time $T$ for which the uniform observability inequality holds.

The proof of Theorem 10 below provides an explicit estimate on the minimal observability time in the class $\mathscr{C}_{h}\left(\gamma / h^{2}\right): T(\gamma)=2 / \sqrt{1-\gamma / 4}$.

Remark 17. In the context of the numerical computation of the boundary control for the wave equation the need of an appropriate filtering of the high frequencies was observed by Glowinski [40] and further investigated numerically by Asch and Lebeau in [2].

Let us now briefly sketch the proof of Theorem 10 The easiest one relies on the explicit representation of the solutions in $\mathscr{C}_{h}\left(\gamma / h^{2}\right)$ and the application of Ingham's theorem. This can be made possible since for all $k$ with $\lambda_{h}^{k} \leq \gamma h^{-2}, \sqrt{\lambda_{h}^{k+1}}-\sqrt{\lambda_{h}^{k}} \geq$ $\pi \cos (k \pi h / 2) \geq \pi \sqrt{1-\gamma / 4}$, as explicit computations yield.

Another proof can be derived using the so-called discrete multiplier identity: for all solutions $\mathbf{u}_{h}$ of (81),

$$
T E_{h}(0)+\left.X_{h}(t)\right|_{0} ^{T}=\frac{1}{2} \int_{0}^{T}\left|\frac{u_{N}(t)}{h}\right|^{2} d t+\frac{h^{3}}{4} \sum_{j=0}^{N} \int_{0}^{T}\left|\frac{u_{j+1}^{\prime}-u_{j}^{\prime}}{h}\right|^{2} d t,
$$

11 These results may also be obtained using discrete multiplier techniques (see [53] and [36] for an improved version with a sharp estimate of the time $T(\delta)$ ). 
with

$$
X_{h}(t)=h \sum_{j=1}^{N} j h\left(\frac{u_{j+1}-u_{j-1}}{2 h}\right) u_{j}^{\prime} .
$$

Using (110) and straightforward bounds on the time boundary term $X_{h}$ and on the extra term

$$
\frac{h^{3}}{4} \sum_{j=0}^{N} \int_{0}^{T}\left|\frac{u_{j+1}^{\prime}-u_{j}^{\prime}}{h}\right|^{2} d t
$$

one will be able to prove Theorem 10 in any time $T>2 /(1-\gamma / 4)$, see [53]. However, using more refined estimates on these terms, one can recover the observability time $T(\gamma)=2 / \sqrt{1-\gamma / 4}$, see [36].

Let us also note that the time $T(\gamma)=2 / \sqrt{1-\gamma / 4}$ is sharp. More precisely, when $T<T(\gamma)$, there is no uniform observability results in the class $\mathscr{C}_{h}\left(\gamma / h^{2}\right)$ since $T(\gamma)$ is the time corresponding to the minimum group velocity within the class $\mathscr{C}_{h}\left(\gamma / h^{2}\right)$. But the proof is technically more involved and is beyond the scope of these notes. We refer to [72] and [36] for detailed proofs.

\subsection{A Two-Grid Algorithm}

Glowinski and $\mathrm{Li}$ in [42] introduced a two-grid algorithm that makes it possible to compute efficiently the control of the continuous model. The method was further developed by Glowinski in [40].

The relevance and impact of using two grids can be easily understood in view of the analysis of the 1-d semidiscrete equation developed in the previous paragraph.

In (88) we have seen that all the eigenvalues of the semidiscrete system satisfy $\lambda \leq 4 / h^{2}$. We have also seen that the observability inequality becomes uniform when one considers solutions involving eigenvectors corresponding to eigenvalues $\lambda \leq \gamma / h^{2}$, with $\gamma<4$, see Theorem 10 .

The key idea of this two-grid filtering mechanism consists in using two grids: one, the computational one in which the discrete wave equations are solved, with step size $h$ and a coarser one of size $2 h$. In the fine grid, the eigenvalues satisfy the sharp upper bound $\lambda \leq 4 / h^{2}$. And the coarse grid will "select" half of the eigenvalues, the ones corresponding to $\lambda \leq 2 / h^{2}$. This indicates that in the fine grid the solutions obtained in the coarse one would behave very much as filtered solutions.

To be more precise, let $N \in \mathbb{N}$ be an odd number, and still consider the semidiscrete wave equation 81 . We then define the class

$$
\begin{aligned}
\mathscr{V}_{h}=\left\{\left(\mathbf{u}_{h}^{0}, \mathbf{u}_{h}^{1}\right) \in \mathbb{R}^{N} \times \mathbb{R}^{N}, \quad u_{2 j+1}^{\ell}\right. & =\frac{u_{2 j}^{\ell}+u_{2 j+2}^{\ell}}{2}, \\
j & \in\{0, \cdots,(N-1) / 2\}, \ell \in\{0,1\}\} .
\end{aligned}
$$


The idea of Glowinski and $\mathrm{Li}$ is then to consider initial data lying in this space, which can be easily described, as we said, in the physical space.

Formally, the oscillations in the coarse mesh that correspond to the largest eigenvalues $\lambda \simeq 4 \sin (\pi / 4)^{2} / h^{2}$, in the finer mesh are associated to eigenvalues in the class of filtered solutions with parameter $\gamma=4 \sin (\pi / 4)^{2}=2$. Formally, this corresponds to a situation where the observability inequality is uniform for $T>2 / \sqrt{1-\gamma / 4}=2 \sqrt{2}$.

The following holds:

Theorem 11. For $N \in \mathbb{N}$ an odd integer and $T>2 \sqrt{2}+2 h$, for any initial data $\left(\mathbf{u}_{h}^{0}, \mathbf{u}_{h}^{1}\right) \in \mathscr{V} h$, the solution $\mathbf{u}_{h}$ of (81) satisfies:

$$
E_{h}(0) \leq \frac{2}{T-2 \sqrt{2}-2 h} \int_{0}^{T}\left|\frac{u_{N}}{h}\right|^{2} d t .
$$

Theorem 11 has been obtained recently in [36] using the multiplier identity (110) and careful estimates on each term in this identity. This approach yields the most explicit estimate on the observability constant for bi-grid techniques.

This issue has also been studied theoretically in the article [83] using the multiplier techniques in 1-d (but getting an observation time $T>4$ ), and later in [50] in $2 \mathrm{~d}$ using a dyadic decomposition argument. The time has later been improved in 1-d to $T>2 \sqrt{2}$ using Ingham techniques in [71], loosing track of the observability constants.

Theorem 11justifies the efficiency of the two-grid algorithm for computing the control of the continuous wave equation, as we shall derive more explicitly in Section 6

This method was introduced by Glowinski [40] in the context of the full finite difference (in time) and finite element space discretization in 2D. It was then further developed in the framework of finite differences by M. Asch and G. Lebeau in [2], where the Geometric Control Condition for the wave equation in different geometries was tested numerically.

\subsection{Tychonoff Regularization}

Glowinski, Li, and Lions [43] proposed a Tychonoff regularization technique that allows one to recover the uniform (with respect to the mesh size) coercivity of the functional that one must minimize to get the controls in the HUM approach. The method was tested to be efficient in numerical experiments.

In the context of observability Tychonoff regularization corresponds to relaxing the boundary observability inequality by adding an extra observation, distributed everywhere in the domain and at the right scale so that it asymptotically vanishes as $h$ tends to zero but it is strong enough to capture the energy of the pathological high frequency components. The corresponding observability inequality is as follows: 


$$
E_{h}(0) \leq C(T)^{2}\left[\int_{0}^{T}\left|\frac{u_{N}(t)}{h}\right|^{2} d t+h^{3} \sum_{j=0}^{N} \int_{0}^{T}\left|\frac{u_{j+1}^{\prime}-u_{j}^{\prime}}{h}\right|^{2} d t\right]
$$

The following holds:

Theorem 12 ([100]). For any time $T>2$, there exists a constant $C(T)$ such that, for all $h>0$, inequality (115) holds for all solutions $\mathbf{u}_{h}$ of 181 . Furthermore, $C(T)^{2}$ can be taken to be $2 /(T-2)$.

In 115 we have the extra term (112) that has already been encountered in the multiplier identity 110. By inspection of the solutions of 81) in separated variables it is easy to understand why this added term is a suitable one to reestablish the uniform observability property. Indeed, consider the solution of the semidiscrete system $\mathbf{u}_{h}=\exp \left( \pm i \sqrt{\lambda_{h}^{k}} t\right) \mathbf{w}_{h}^{k}$. The extra term we have added is of the order of $h^{2} \lambda_{h}^{k} E_{h}(0)$. Obviously this term is negligible as $h \rightarrow 0$ for the low-frequency solutions (for $k$ fixed) but becomes relevant for the high-frequency ones when $\lambda_{h}^{k} \sim 1 / h^{2}$. Accordingly, when inequality (86) fails, i.e., for the high-frequency solutions, the extra term in (115) reestablishes the uniform character of the estimate with respect to $h$. It is important to emphasize that both terms are needed for 115 to hold. Indeed, 112 by itself does not suffice since its contribution vanishes as $h \rightarrow 0$ for the low-frequency solutions.

We do not give the proof of Theorem 12, which is an easy consequence of the discrete multiplier identity 110-111.

\subsection{Space semidiscretizations of the 2D Wave Equations}

In this Section we briefly discuss the results in [111] on the space finite difference semidiscretizations of the $2 \mathrm{D}$ wave equation in the square $\Omega=(0, \pi) \times(0, \pi)$ of $\mathbb{R}^{2}$ :

$$
\begin{cases}u_{t t}-\Delta u=0 & \text { in } Q=\Omega \times(0, T), \\ u=0 & \text { on } \partial \Omega \times(0, T), \\ u(x, 0)=u^{0}(x), u_{t}(x, 0)=u^{1}(x) & \text { in } \Omega .\end{cases}
$$

Obviously, the fact that classical finite differences provide divergent results for 1$\mathrm{d}$ problems in what concerns observability and controllability indicates that the same should be true in two dimensions as well. This is indeed the case. However, the multidimensional case exhibits some new features and deserves additional analysis, in particular in what concerns filtering techniques. Given $\left(u^{0}, u^{1}\right) \in H_{0}^{1}(\Omega) \times L^{2}(\Omega)$, system (116) admits a unique solution $u \in C\left([0, T] ; H_{0}^{1}(\Omega)\right) \cap C^{1}\left([0, T] ; L^{2}(\Omega)\right)$. Moreover, the energy

$$
E(t)=\frac{1}{2} \int_{\Omega}\left[\left|u_{t}(x, t)\right|^{2}+|\nabla u(x, t)|^{2}\right] d x
$$


remains constant, i.e.,

$$
E(t)=E(0) \quad \forall 0<t<T .
$$

Let $\Gamma$ denote a subset of the boundary of $\Omega$ constituted by two consecutive sides, for instance,

$$
\Gamma=\left\{\left(x_{1}, \pi\right): x_{1} \in(0, \pi)\right\} \cup\left\{\left(\pi, x_{2}\right): x_{2} \in(0, \pi)\right\} .
$$

It is well known (see [68, 69]) that for $T>2 \sqrt{2} \pi$ there exists $C_{o b s}(T)>0$ such that

$$
E(0) \leq C_{o b s}(T)^{2} \int_{0}^{T} \int_{\Gamma}\left|\frac{\partial u}{\partial n}\right|^{2} d \sigma d t
$$

holds for every finite-energy solution of (116).

We can now address the standard five-point finite difference space semidiscretization scheme for the 2-d wave equation.

As in one dimension we may perform a complete description of both the continuous solutions and those of the semidiscrete systems in terms of Fourier series. One can then deduce the following:

- The semidiscrete system is observable for all time $T$ and mesh size $h$;

- The observability constant $C_{h}(T)$ blows up as $h$ tends to 0 because of the spurious high-frequency numerical solutions.

- The uniform (with respect to $h$ ) observability property may be reestablished by a suitable filtering of the high frequencies.

However, filtering needs to be implemented more carefully in the multi-dimensional case.

Indeed, the upper bound on the spectrum of the semidiscrete system in two dimensions is $8 / h^{2}$ but it is not sufficient to filter by a constant $0<\gamma<8$, i.e., to consider solutions that do not contain the contribution of the high frequencies $\lambda>\gamma h^{-2}$, to guarantee uniform observability.

In fact, one has to filter by means of a constant $0<\gamma<4$. This is due to the existence of solutions corresponding to high-frequency oscillations in one direction and very slow oscillations in the other. Roughly speaking, one needs to filter efficiently in both space directions, and this requires taking $\gamma<4$ (see [111]).

In order to better understand the necessity of filtering and getting sharp observability times it is convenient to adopt the approach of [72, 73] based on the use of discrete Wigner measures. The symbol of the semidiscrete system for solutions of wavelength $h$ is

$$
\tau^{2}-4\left(\sin ^{2}\left(\xi_{1} / 2\right)+\sin ^{2}\left(\xi_{2} / 2\right)\right)
$$

and can be easily obtained as in the von Neumann analysis of the stability of numerical schemes by taking the Fourier transform of the semidiscrete equation: the continuous one in time and the discrete one in space 12

12 This argument can be easily adapted to the case where the numerical approximation scheme is discrete in both space and time by taking discrete Fourier transforms in both variables. 
Note that in the symbol in 121) the parameter $h$ disappears. This is due to the fact that we are analyzing the propagation of waves of wavelength of the order of $h$.

The bicharacteristic rays are then defined as follows:

$$
\left\{\begin{array}{l}
x_{j}^{\prime}(s)=-2 \sin \left(\xi_{j} / 2\right) \cos \left(\xi_{j} / 2\right)=-\sin \left(\xi_{j}\right), \quad j=1,2, \\
t^{\prime}(s)=\tau, \\
\xi_{j}^{\prime}(s)=0, \quad j=1,2, \\
\tau^{\prime}(s)=0 .
\end{array}\right.
$$

It is interesting to note that the rays are still straight lines, as for the constant coefficient wave equation, since the coefficients of the equation and the numerical discretization are both constant. We see, however, that in (122) the velocity of propagation changes with respect to that of the continuous wave equation.

Let us now consider initial data for this Hamiltonian system with the following particular structure: $x^{0}$ is any point in the domain $\Omega$, the initial time $t_{0}=0$, and the initial microlocal direction $\left(\tau^{*}, \xi^{*}\right)$ is such that

$$
\left(\tau^{*}\right)^{2}=4\left(\sin ^{2}\left(\xi_{1}^{*} / 2\right)+\sin ^{2}\left(\xi_{2}^{*} / 2\right)\right) .
$$

Note that the last condition is compatible with the choice $\xi_{1}^{*}=0$ and $\xi_{2}^{*}=\pi$ together with $\tau^{*}=2$. Thus, let us consider the initial microlocal direction $\xi_{2}^{*}=\pi$ and $\tau^{*}=2$. In this case the ray remains constant in time, $x(t)=x^{0}$, since, according to the first equation in (122), $x_{j}^{\prime}$ vanishes both for $j=1$ and $j=2$. Thus, the projection of the ray over the space $x$ does not move as time evolves. This ray never reaches the exterior boundary $\partial \Omega$ where the equation evolves and excludes the possibility of having a uniform boundary observability property. More precisely, this construction allows one to show that, as $h \rightarrow 0$, there exists a sequence of solutions of the semidiscrete problem whose energy is concentrated in any finite time interval $0 \leq t \leq T$ as much as one wishes in a neighborhood of the point $x^{0}$.

This example corresponds to the case of very slow oscillations in the space variable $x_{1}$ and very rapid ones in the $x_{2}$-direction, and it can be ruled out, precisely, by taking the filtering parameter $\gamma<4$. In view of the structure of the Hamiltonian system, it is clear that one can be more precise when choosing the space of filtered solutions. Indeed, it is sufficient to exclude by filtering the rays that do not propagate at all to guarantee the existence of a minimal velocity of propagation (see Figure 10). Roughly speaking, this suffices for the observability inequality to hold uniformly in $h$ for a sufficiently large time [72, 73].

This ray approach makes it possible to conjecture the optimal uniform observability time depending on the class of filtered solutions under consideration. The optimal time is the one that all characteristic rays entering in the class of filtered solutions need to reach the controlled region. This constitutes the discrete version of the GCC for the continuous wave equation. Moreover, if the filtering is done so that the wavelength of the solutions under consideration is of an order strictly less than $h$, then one recovers the classical observability result for the constant coefficient continuous wave equation with the optimal observability time. 


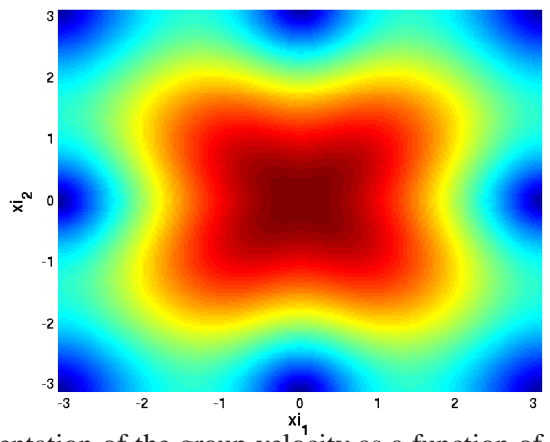

Fig. 10 Level set representation of the group velocity as a function of the frequency $\left(h \xi_{1}, h \xi_{2}\right) \in$ $(-\pi, \pi)$. In red, the points where the group velocity is 1 , which is the velocity of propagation of continuous waves. In blue, the points where the group velocity is close to zero. When, by means of a filtering method the blue areas are removed, the velocity of propagation of rays is uniformly bounded from below.

\subsection{A more general result}

Here, we describe the most general result available in the literature for uniform observability of space semidiscrete wave equations.

This concerns the finite-element discretization of (59) observed through some subdomain $\omega$. Let us emphasize from the beginning that the results presented in that Section hold under the Geometric Control Condition for $(\Omega, \omega, T)$, whatever the dimension is and under very mild assumptions on the finite-element discretization under consideration.

In the following, to simplify the presentation, we focus on the constant coefficient wave equation:

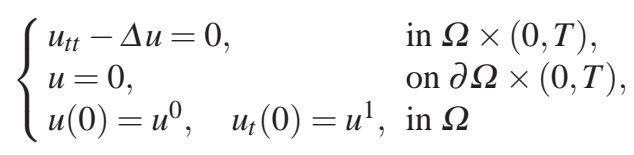

observed through $\chi_{\omega} u_{t}$ on $\omega \times(0, T)$.

The corresponding observability inequality is

$$
\left\|\nabla u^{0}\right\|_{L^{2}(\Omega)}^{2}+\left\|u^{1}\right\|_{L^{2}(\Omega)}^{2} \leq C_{o b s}^{2} \int_{0}^{T}\left\|\chi_{\omega} u_{t}(t)\right\|_{L^{2}(\Omega)}^{2} d t .
$$

Let us now describe the finite element method we use to discretize (124).

Consider $\left(V_{h}\right)_{h>0}$ a sequence of vector spaces of finite dimension $n_{h}$ that embed $V_{h}$ into $L^{2}(\Omega)$ using a linear morphism $\rho_{h}: V_{h} \rightarrow L^{2}$. For each $h>0$, the inner product $\langle\cdot, \cdot\rangle_{L^{2}}$ in $L^{2}$ induces a structure of Hilbert space for $V_{h}$ endowed by the scalar product $\langle\cdot, \cdot\rangle_{h}=\left\langle\rho_{h^{\cdot}}, \rho_{h^{\cdot}}\right\rangle_{L^{2}}$. We assume that for each $h>0$, the vector space $\rho_{h}\left(V_{h}\right)$ is a subspace of $\mathscr{D}\left(\left(-\Delta_{D}\right)^{1 / 2}\right)=H_{0}^{1}(\Omega)$. We thus define the linear operator 
$A_{0 h}: V_{h} \rightarrow V_{h}$ by

$$
\left\langle A_{0 h} \phi_{h}, \psi_{h}\right\rangle_{h}=\left\langle\nabla \rho_{h} \phi_{h}, \nabla \rho_{h} \psi_{h}\right\rangle_{L^{2}}, \quad \forall\left(\phi_{h}, \psi_{h}\right) \in V_{h}^{2} .
$$

The operator $A_{0 h}$ defined in (126) obviously is self-adjoint and positive definite.Formally, definition (126) implies that

$$
A_{0 h}=\left(\nabla \rho_{h}\right)^{*} \nabla \rho_{h} .
$$

This operator $A_{0 h}$ corresponds to the finite element discretization of $-\Delta_{D}$, the Laplace operator with Dirichlet boundary conditions. System (124) is then discretized into

$$
\mathbf{u}_{h}^{\prime \prime}+A_{0 h} \mathbf{u}_{h}=0, \quad \mathbf{u}_{h}(0)=\mathbf{u}_{h}^{0} \in V_{h}, \quad \mathbf{u}_{h}^{\prime}(0)=\mathbf{u}_{h}^{1} \in V_{h} .
$$

In this context, for all $h>0$, the observation operator naturally becomes $\chi_{\omega} \rho_{h} u_{h}^{\prime}(t)$.

We now make precise the assumptions we have, usually, on $\rho_{h}$, and which will be needed in our analysis. For this, we introduce the adjoint of $\rho_{h}$ from $V_{h}$ endowed with the scalar product of $\left\langle A_{0 h}^{1 / 2} \cdot, A_{0 h}^{1 / 2} \cdot\right\rangle_{h}$ to $\mathscr{D}\left(A_{0}^{1 / 2}\right)=H_{0}^{1}(\Omega)$ endowed with the scalar product $\langle\nabla \cdot, \nabla \cdot\rangle_{L^{2}}$.

One easily checks that $\rho_{h}^{*} \rho_{h}=I d_{V_{h}}$. Besides, the embedding $\rho_{h}$ describes the finite element approximation we have chosen. In particular, the vector space $\rho_{h}\left(V_{h}\right)$ approximates, in the sense given hereafter, the space $\mathscr{D}\left(A_{0}^{1 / 2}\right)=H_{0}^{1}(\Omega)$ : There exist $\theta>0$ and $C_{0}>0$, such that for all $h>0$,

$$
\left\{\begin{array}{l}
\left\|\nabla\left(\rho_{h} \rho_{h}^{*}-I\right) u\right\|_{L^{2}(\Omega)} \leq C_{0}\|\nabla u\|_{L^{2}(\Omega)}, \quad \forall u \in H_{0}^{1}(\Omega), \\
\left\|\nabla\left(\rho_{h} \rho_{h}^{*}-I\right) u\right\|_{L^{2}(\Omega)} \leq C_{0} h^{\theta}\|-\Delta u\|_{L^{2}(\Omega)}, \quad \forall u \in H^{2} \cap H_{0}^{1}(\Omega) .
\end{array}\right.
$$

Note that in many applications, estimates (129) are satisfied for $\theta=1$. This is in particular true when discretizing on uniformly regular meshes (see [92]).

We will not discuss convergence results for the numerical approximation schemes presented here, which are classical under assumption (129), and which can be found for instance in the textbook [92].

In view of the previous results, it is natural to restrict ourselves to filtered initial data. For all $h>0$, since $A_{0 h}$ is a self adjoint positive definite matrix, the spectrum of $A_{0 h}$ is given by a sequence of positive eigenvalues

$$
0<\lambda_{h}^{1} \leq \lambda_{h}^{2} \leq \cdots \leq \lambda_{h}^{n_{h}}
$$

and normalized (in $V_{h}$ ) eigenvectors $\left(\mathbf{w}_{h}^{k}\right)_{1 \leq k \leq n_{h}}$. For any $s$, we can now define, for each $h>0$, the filtered space (to be compared with (108))

$$
\mathscr{C}_{h}(s)=\left\{\mathbf{u}_{h}=\sum_{\lambda_{h}^{k} \leq s}\left(a_{k} \cos \left(\sqrt{\lambda_{h}^{k}} t\right)+\frac{b_{k}}{\sqrt{\lambda_{h}^{k}}} \sin \left(\sqrt{\lambda_{h}^{k}} t\right)\right) \mathbf{w}_{h}^{k}\right\} .
$$


We are now in position to state the following results:

Theorem 13 ([27]). Assume that the maps $\left(\rho_{h}\right)_{h>0}$ satisfy property (129) and that $(\omega, \Omega, T)$ satisfies the Geometric Control Condition, i.e. that system (124) is exactly observable.

Then there exist $\varepsilon>0$, a time $T^{*}$ and a positive constant $C_{\text {obs }}$ such that, for any $h \in(0,1)$, any solution of (128) lying in $\mathscr{C}_{h}\left(\varepsilon / h^{\theta}\right)$ satisfies

$$
\left\|\nabla \rho_{h} \mathbf{u}_{h}^{0}\right\|_{L^{2}(\Omega)}^{2}+\left\|\rho_{h} \mathbf{u}_{h}^{1}\right\|_{L^{2}(\Omega)}^{2} \leq C_{o b s}^{2} \int_{0}^{T^{*}}\left\|\chi_{\omega} \rho_{h} \mathbf{u}_{h}^{\prime}(t)\right\|_{L^{2}(\Omega)}^{2} d t .
$$

Note in particular that this yields the same results as the one obtained in [90] in a 1-d framework and generalizes it to any dimension.

The proof of this Theorem combines, essentially, the observability inequality of the continuous wave equation and sharp estimates on the convergence of the numerical scheme towards the continuous model. Roughly speaking, one needs to build the subspace of initial data so that numerical solutions are uniformly close to the continuous ones so that they inherit the observability properties of the later.

The interest of this result is that it holds in any space dimension and in a very general Galerkin approximation setting. To our knowledge, [27] and the companion paper [29] are the first ones in which this kind of results are presented with such a degree of generality.

The proof of this statement can be derived using resolvent estimates [16, 79] (see also [88] for a similar estimate) but this method does not yield sharp estimates on the observability time. Hence $T^{*}$ in Theorem 13 may be much larger than the time for which $(\omega, \Omega, T)$ satisfies GCC and the one one could expect to be sharp in view of the analysis of the dispersion diagram of the numerical scheme.

Note also that (131) holds within a class of functions that are much more filtered than in Theorem 10. The later holds up to the critical scale within subclasses of the form $\mathscr{C}_{h}\left(\gamma / h^{2}\right), \gamma<4$. Whether the result in Theorem 13 is true or not in these optimal subclasses is an interesting open problem. Note, in any case, that Theorem 13 holds in a much more general setting, where new phenomena could occur. Even in 1-d, for the finite element method on non-uniform meshes, whether Theorem 13 can be improved or not is an open problem.

\section{Convergence results}

The goal of this Section is to describe a general approach to show the convergence of the discrete controls, obtaining convergence rates, from the observability results presented in the previous Section. 


\subsection{A general procedure for the convergence of the discrete controls}

In this Section, we describe the setting in which we are working, and present the main ideas.

Let $A$ be a skew-adjoint operator $A: \mathscr{D}(A) \subset X \rightarrow X$ with compact resolvent and dense domain, and $B$ be an admissible control operator $B \in \mathfrak{L}\left(\mathscr{U}, X_{-1}\right)$.

We assume that the continuous system (13) is controllable in some time $T>0$.

Now, we approximate the continuous model (13) by a sequence of finite-dimensional systems

$$
\mathbf{x}_{h}^{\prime}=A_{h} \mathbf{x}_{h}+B_{h} \mathbf{v}_{h}, \quad t \geq 0, \quad \mathbf{x}_{h}(0)=\mathbf{x}_{h}^{0} \in X_{h},
$$

where $\left(A_{h}, B_{h}\right)$ is a sequence of finite-dimensional approximations of the operators $(A, B)$ respectively, where for each $h>0, A_{h}$ is a skew-adjoint operator defined on a finite dimensional space $X_{h}$ embedded into $X$, and $B_{h}$ is defined on a vector space $\mathscr{U}_{h}$ that embeds into the Hilbert space $\mathscr{U}$ with values in $X_{h}$.

We consider the embedding $\rho_{h}: X_{h} \rightarrow X$, which provides an Hilbert structure on $X_{h}$ by $\|\cdot\|_{h}=\left\|\rho_{h}(\cdot)\right\|_{X}$.

To simplify the presentation, we further assume that $B_{h}$ is simply given by $\rho_{h}^{*} B$, where $B$ is the continuous control operator, so that $\mathscr{U}_{h}$ simply coincides with $\mathscr{U}$. Otherwise, similar ideas can be applied, see for instance Section 6.2.3

We also assume that the spaces $X_{h}$ fill the space $X$ as $h \rightarrow 0$ in a sense that will be made precise below. Of course, the finite difference or the finite-element approximation schemes for the wave equation fit into this setting, and a more precise description can be made in these cases.

We have already seen in Section 4.4 that, for the finite-difference method, the discrete controls fulfilling the control requirement $\mathbf{x}_{h}(T)=0$ may blow up as $h \rightarrow 0$, due to the fact that observability properties do not hold uniformly with respect to the discretization parameter $h>0$.

However, we have seen in Section 5 that weak observability results can be shown to hold uniformly with respect to the discretization parameter $h>0$, provided suitable filtering mechanisms are implemented. To be more precise, we assume that there exist a positive constant $C_{o b s}$ and a time $T$ such that, for all $h>0$,

$$
\left\|\varphi_{h}^{T}\right\|_{h}^{2} \leq C_{o b s}^{2} \int_{0}^{T} \eta(t)\left\|B_{h}^{*} \varphi_{h}(t)\right\|_{\mathscr{U}}^{2} d t, \quad \forall \varphi_{h}^{T} \in \mathfrak{C}_{h},
$$

where $\mathfrak{C}_{h}$ is a subspace of $X_{h}, \eta$ is a smooth function with values in $[0,1]$, vanishing for $t \notin[0, T]$ and equals to 1 on some non trivial subset of $[0, T]$, similarly as in [21), and $\varphi_{h}$ is the solution of the adjoint system

$$
\varphi_{h}^{\prime}=A_{h} \varphi_{h}, \quad t \in(0, T), \quad \varphi_{h}(T)=\varphi_{h}^{T} .
$$

We now consider the HUM-type functional $J_{h}$, defined for $\varphi_{h}^{T} \in \mathfrak{C}_{h}$ by 


$$
J_{h}\left(\varphi_{h}^{T}\right)=\frac{1}{2} \int_{0}^{T} \eta(t)\left\|B_{h}^{*} \varphi_{h}(t)\right\|_{\mathscr{U}}^{2} d t+\left\langle\mathbf{x}_{h}^{0}, \varphi_{h}(0)\right\rangle_{h} .
$$

Using the same arguments as in Theorem 1 and Corollary 1, one easily checks that:

Theorem 14. Assume that (133) holds. with constants $C$ and $T$ independent of $h>$ 0 .

Let $h>0$ and $\mathbf{x}_{h}^{0} \in X_{h}$. Then the functional $J_{h}$ in (135) is continuous, strictly convex and coercive on $\mathfrak{C}_{h}$ and it admits a unique minimizer $\Phi_{h}^{T} \in \mathfrak{C}_{h}$. Then, setting $\mathbf{V}_{h}=\eta(t) B_{h}^{*} \Phi_{h}$, where $\Phi_{h}$ is the solution of (134) with initial data $\Phi_{h}^{T}$, the solution $\mathbf{x}_{h}$ of (132) satisfies

$$
\forall \varphi_{h}^{T} \in \mathfrak{C}_{h}, \quad\left\langle\varphi_{h}^{T}, \mathbf{x}_{h}(T)\right\rangle_{h}=0,
$$

or equivalently $x_{h}(T) \in \mathfrak{C}_{h}^{\perp}$.

Besides, this is the only control $\mathbf{V}_{h} \in L^{2}(0, T ; \mathscr{U})$ such that the corresponding $\mathbf{x}_{h}$ satisfies (136) and which is of the form $\mathbf{V}_{h}=\eta B_{h}^{*} \varphi_{h}$ for some $\varphi_{h}$ solution of (134) with $\varphi_{h}(T) \in \mathfrak{C}_{h}$.

Moreover,

$$
\frac{1}{C_{o b s}^{2}}\left\|\Phi_{h}^{T}\right\|_{h}^{2} \leq \int_{0}^{T}\left\|\mathbf{V}_{h}(t)\right\|_{\mathscr{U}}^{2} \frac{d t}{\eta(t)}=\int_{0}^{T} \eta(t)\left\|B_{h}^{*} \Phi_{h}(t)\right\|_{\mathscr{U}}^{2} d t \leq C_{o b s}^{2}\left\|\mathbf{x}_{h}^{0}\right\|_{h}^{2} .
$$

The following two questions arise now naturally:

- Convergence. Given $\mathbf{x}_{h}^{0}$ that converge (weakly or strongly) to $x^{0}$ in $X$ as $h \rightarrow 0$ (in a sense to be made precise), can we show that the discrete controls $\mathbf{V}_{h}$ converge to $V$, the continuous control corresponding to $x^{0}$ for (13) ?

- Convergence rates. Can we furthermore give a convergence rate for the convergence of $\mathbf{V}_{h}$ towards $V$ ?

These two questions will be investigated below in this very general setting. Of course, getting such results requires a more precise knowledge of the numerical schemes under consideration.

We shall then present a general frame on which, under suitable hypotheses that should then be carefully verified in each situation, the convergence will be proved with convergence rates.

\subsubsection{Convergence}

To derive the convergence of the discrete controls $\mathbf{V}_{h}$ given by Theorem 14 we need the following hypotheses, that should be verified in each situation:

Hypothesis \#1. For $\varphi^{T} \in \cap_{s>0} \mathscr{D}\left(A^{s}\right)$ and $\varphi$ be the corresponding solution of (14), there exists a sequence of functions $\varphi_{h}^{T} \in \mathfrak{C}_{h}$ such that, if $\varphi_{h}$ denotes the corresponding solution of 134, 


$$
\begin{aligned}
& \rho_{h} \varphi_{h}(0) \underset{h \rightarrow 0}{\longrightarrow} \varphi(0) X \\
& B_{h}^{*} \varphi_{h} \underset{h \rightarrow 0}{\longrightarrow} B^{*} \varphi \quad L^{2}(0, T ; \mathscr{U}) .
\end{aligned}
$$

Hypothesis \#1 looks like a classical result of convergence of the numerical methods under consideration. This is indeed the case, except for the fact that the approximations of $\varphi^{T}$ are searched within the restricted subspace $\mathfrak{C}_{h}$ of $X_{h}$. This in practice requires proving the convergence of suitable projections of the numerical approximations.

We also need the following assumption:

Hypothesis \#2. For $\varphi_{h}^{T} \in X_{h}$ and $\varphi^{T} \in X$ such that

$$
\rho_{h} \varphi_{h}^{T} \underset{h \rightarrow 0}{\rightarrow} \varphi^{T} \quad X \quad \text { and } \sup _{h}\left\|B_{h}^{*} \varphi_{h}(t)\right\|_{L^{2}(0, T ; U)}<\infty,
$$

denoting by $\varphi_{h}$ and $\varphi$ respectively the solutions of (134) and (14) with initial data $\varphi_{h}^{T}$ and $\varphi^{T}$ respectively,

$$
\begin{gathered}
\rho_{h} \varphi_{h} \underset{h \rightarrow 0}{\rightarrow} \varphi \quad L^{2}(0, T ; X) \\
B_{h}^{*} \varphi_{h} \underset{h \rightarrow 0}{\rightarrow} B^{*} \varphi \quad L^{2}(0, T ; \mathscr{U}) \\
\rho_{h} \varphi_{h}(0) \underset{h \rightarrow 0}{\rightarrow} \varphi(0) \quad X .
\end{gathered}
$$

The statements in Hypothesis \#2 typically hold for classical numerical approximation schemes.

Under these two main hypotheses we get the following result:

Theorem 15. Let $x^{0} \in X$ and $\mathbf{x}_{h}^{0} \in X_{h}$ be such that $\rho_{h} \mathbf{x}_{h}^{0}$ weakly converges to $x^{0}$ in $X$ as $h \rightarrow 0$.

We further assume that Hypotheses \#1 and \#2 hold true.

Then the discrete controls $\mathbf{V}_{h}$ given by Theorem 14 weakly converge to $V$ given by Proposition $\square$ in $L^{2}(0, T ; d t / \eta ; \mathscr{U})$ as $h \rightarrow 0$.

Moreover, if $\rho_{h} \mathbf{x}_{h}^{0}$ strongly converge to $x^{0}, \mathbf{V}_{h}$ strongly converge to $V$ in the norm of $L^{2}(0, T ; d t / \eta ; \mathscr{U})$ (hence in the $L^{2}(0, T ; \mathscr{U})$-norm as well) as $h \rightarrow 0$.

Proof. The proof of Theorem 15 is divided into several steps.

Step 1. Extraction of a weakly convergent sequence of controls. From Theorem 14 the sequence $\mathbf{V}_{h}$ is bounded in $L^{2}(0, T ; d t / \eta ; \mathscr{U})$. Hence, up to extraction of a subsequence, the controls $\mathbf{V}_{h}$ weakly converge to some function $v$ in $L^{2}(0, T ; d t / \eta ; \mathscr{U})$.

Step 2. Any weak accumulation point of $\mathbf{V}_{h}$ is a control function for (13). The Euler-Lagrange equation satisfied by the minimizer $\Phi_{h}^{T}$ of $J_{h}$ in (135) is the following one:

$$
\forall \varphi_{h}^{T} \in \mathfrak{C}_{h}, \quad \int_{0}^{T}\left\langle\mathbf{V}_{h}(t), B_{h}^{*} \varphi_{h}\right\rangle_{\mathscr{U}} d t+\left\langle\mathbf{x}_{h}^{0}, \varphi_{h}(0)\right\rangle_{h}=0 .
$$


Let us then take $\varphi^{T} \in \cap_{s>0} \mathscr{D}\left(A^{s}\right)$. Using Hypothesis \#1, we obtain a sequence $\varphi_{h}^{T} \in$ $\mathfrak{C}_{h}$ such that the strong convergences 1139 - $(138)$ hold. Further using that

$$
\left\langle\mathbf{x}_{h}^{0}, \varphi_{h}(0)\right\rangle_{h}=\left\langle\rho_{h} \mathbf{x}_{h}^{0}, \rho_{h} \varphi_{h}(0)\right\rangle_{X},
$$

and passing to the limit in (144), we obtain that for all $\varphi^{T} \in \cap_{s>0} \mathscr{D}\left(A^{s}\right)$,

$$
\int_{0}^{T}\left\langle v(t), B^{*} \varphi\right\rangle_{\mathscr{U}} d t+\left\langle x^{0}, \varphi(0)\right\rangle_{X}=0
$$

By density, this also holds true for all $\varphi^{T} \in X$. From (20), this implies that $v$ is a control function for (13).

Step 3. Any weak accumulation point $v$ of $\mathbf{V}_{h}$ can be written as $v=\eta B^{*} \varphi$ for some $\varphi$ solution of the adjoint system (14). For all $h>0, \mathbf{V}_{h}=\eta B_{h}^{*} \Phi_{h}$, where $\Phi_{h}$ is the solution of (134) with initial data $\Phi_{h}^{T}$, and $\mathbf{V}_{h}$ and $\rho_{h} \Phi_{h}^{T}$ are bounded, respectively, in $L^{2}(0, T ; d t / \eta ; \mathscr{U})$ and $X$, due to (137). Thus, up to subsequence, $\rho_{h} \Phi_{h}^{T}$ weakly converge in $X$ to some $\varphi^{T}$. Thus, from Hypothesis \#2, $v=\eta B^{*} \varphi$, where $\varphi$ is the solution of (14) corresponding to $\varphi^{T}$.

Step 4. Any weak accumulation point of $\mathbf{V}_{h}$ is the control $V$ given by Proposition 17 This follows from the uniqueness of the control functions that can be written $\eta B^{*} \varphi$ for some $\varphi$ solution of (14) (see Proposition 1).

Hence there is only one weak accumulation point for the sequence $\left(\mathbf{V}_{h}\right)$, which coincides with the control $V$ given by Proposition 1 . Therefore, the sequence $\left(\mathbf{V}_{h}\right)$ weakly converges to $V$ in $L^{2}(0, T ; d t / \eta ; \mathscr{U})$ as $h \rightarrow 0$.

Step 5. Strong convergence when $\rho_{h} \mathbf{x}_{h}^{0}$ strongly converges to $x^{0}$. In view of the weak convergence property from Step 4 , we only need to prove the convergence of the $L^{2}(0, T ; d t / \eta ; \mathscr{U})$-norms of $\mathbf{V}_{h}$ as $h \rightarrow 0$.

But, from (144) applied to $\Phi_{h}^{T}\left(\in \mathfrak{C}_{h}\right)$,

$$
\left\|\mathbf{V}_{h}\right\|_{L^{2}(0, T ; d t / \eta ; \mathscr{U})}^{2}=\int_{0}^{T} \eta(t)\left\|B_{h}^{*} \Phi_{h}(t)\right\|_{\mathscr{U}}^{2} d t=-\left\langle\rho_{h} \mathbf{x}_{h}^{0}, \rho_{h} \Phi_{h}(0)\right\rangle_{X} .
$$

On the other hand, $V=\eta B^{*} \Phi$, where $\Phi$ is given by Proposition 1]. From (20) applied to $\varphi^{T}=\Phi^{T}$, we obtain

$$
\|V\|_{L^{2}(0, T ; d t / \eta ; \mathscr{U})}^{2}=\int_{0}^{T} \eta(t)\left\|B^{*} \Phi(t)\right\|_{\mathscr{U}}^{2} d t=-\left\langle x_{0}, \Phi(0)\right\rangle_{X} .
$$

Now, using Step 3 and Hypothesis \#2, $\rho_{h} \Phi_{h}^{T}$ weakly converges to some $\varphi^{T}$ in $X$ which is such that $V=\eta B^{*} \varphi$. From the observability inequality (22), $\varphi \equiv \Phi$, the one corresponding to the minimizer of the functional $J$ in $(23)$. Hence $\rho_{h} \Phi_{h}^{T}$ weakly converges in $X$ to $\Phi^{T}$. Applying again Hypothesis \#2, $\rho_{h} \Phi_{h}(0)$ weakly converges to $\Phi(0)$ in $X$ as $h \rightarrow 0$.

Passing to the limit, $\left\langle\rho_{h} \mathbf{x}_{h}^{0}, \rho_{h} \Phi_{h}(0)\right\rangle_{X}$ converges to $\left\langle x_{0}, \Phi(0)\right\rangle_{X}$ as $h \rightarrow 0$, and then passing to the limit in (146) and using (147), the $L^{2}(0, T ; d t / \eta ; \mathscr{U})$-norms of $\mathbf{V}_{h}$ converge to the $L^{2}(0, T ; d t / \eta ; \mathscr{U})$-norm of $V$.

This concludes the proof of the Theorem. 
Note that this method of proof is not new (see, for instance, [53]) and it has been shown to be robust and efficient, whatever the discretization scheme or the weak observability properties under consideration are.

However, this approach did not seem to be sufficient to get convergence rates for the discrete controls. The main reason is that it was not known, with this degree of generality, that smooth initial data to be controlled yield smooth controls. As we have explained above, we now know that such results hold true in a broad abstract setting, but only when the cut-off function in time $\eta(t)$ is introduced or when the control operator is bounded, i.e. $B \in \mathfrak{L}(\mathscr{U}, X)$. Then, using Theorem 3 , we will be in conditions to prove also convergence rates.

\subsubsection{Convergence rates}

To prove convergence rates for the discrete controls towards the continuous ones, it is necessary, as is standard in numerical analysis, to assume some smoothness on the initial data. One then needs to make sure that the numerical schemes approximating the PDE model have suitable convergence rates that we will then transfer to the controls. In the following Hypothesis \#3 we require this property to be fulfilled.

Hypothesis \#3. There exist $s_{1}>0$ and a constant $\theta_{1}>0$ such that for all $\varphi^{T} \in$ $\mathscr{D}\left(A^{s_{1}}\right)$, one can find a sequence of functions $\varphi_{h}^{T} \in \mathfrak{C}_{h}$ such that the corresponding solutions $\varphi_{h}$ of (134) satisfy, for $h>0$,

$$
\sup _{t \in(0, T)}\left(\left\|\rho_{h} \varphi_{h}-\varphi\right\|_{X}\right)+\left\|B^{*}\left(\rho_{h} \varphi_{h}-\varphi\right)\right\|_{L^{2}(0, T ; \mathscr{U})} \leq C h^{\theta_{1}}\left\|\varphi^{T}\right\|_{\mathscr{D}\left(A^{s_{1}}\right)},
$$

where $\varphi$ is the solution of (14) with initial data $\varphi^{T}$.

Note that Hypothesis \#3 is a stronger version of Hypothesis \#1. It always holds with $X_{h}$ instead of $\mathfrak{C}_{h}$ for convergent numerical approximation schemes. As we shall see, in specific examples, similar results hold within the classes $\mathfrak{C}_{h}$ as assumed in Hypothesis \#3.

Also note that when $B^{*}$ is bounded, estimate (148) is implied by the weaker one:

$$
\sup _{t \in(0, T)}\left\|\rho_{h} \varphi_{h}-\varphi\right\|_{X} \leq C h^{\theta_{1}}\left\|\varphi^{T}\right\|_{\mathscr{D}\left(A^{s_{1}}\right)} .
$$

We also need a similar convergence assumption for the controlled equation:

Hypothesis \#4. There exist $s_{2}>0$ and a constant $\theta_{2}$ such that for all $x^{0} \in \mathscr{D}\left(A^{s_{2}}\right)$ and $\Phi^{T} \in \mathscr{D}\left(A^{s_{2}}\right)$, setting $\mathbf{x}_{h}^{0}=\rho_{h}^{*} x^{0}, v=\eta B^{*} \Phi$ where $\Phi$ is the solution of (14) with initial data $\Phi^{T}$ and $\mathbf{v}_{h} \in L^{2}(0, T ; \mathscr{U})$, the corresponding solutions $\mathbf{x}_{h}$ and $x$ of (132) and (13) respectively satisfy:

$$
\left\|\rho_{h} \mathbf{x}_{h}(T)-x(T)\right\|_{X} \leq C h^{\theta_{2}}\left(\left\|x^{0}\right\|_{\mathscr{D}\left(A^{s_{2}}\right)}+\left\|\Phi^{T}\right\|_{\mathscr{D}\left(A^{s_{2}}\right)}\right)+C\left\|\mathbf{v}_{h}-v\right\|_{L^{2}(0, T ; \mathscr{U})} .
$$


Note that Hypothesis \#4 looks like a classical convergence result for numerical methods. The fact that the source term is given as $\eta B^{*} \Phi$ is needed to guarantee that the controlled trajectory $x$ lies in a smooth space, and in particular that this is a strong solution, see Corollary 3 and Section 3.3

We are now in position to state our main result:

Theorem 16. Assume that Hypotheses \#3 and \#4 hold.

Let $s=\max \left\{s_{1}, s_{2}\right\}$ and $\theta=\min \left\{\theta_{1}, \theta_{2}\right\}$.

Then, for any $x^{0} \in \mathscr{D}\left(A^{s}\right)$, setting $\mathbf{x}_{h}^{0}=\rho_{h}^{*} x^{0}$, the discrete controls $\mathbf{V}_{h}$ given by Theorem 14 converge to the control $V$ given by Proposition $\square$ and

$$
\left\|\mathbf{V}_{h}-V\right\|_{L^{2}(0, T ; d t / \eta ; \mathscr{U})} \leq C h^{\theta}\left\|x^{0}\right\|_{\mathscr{D}\left(A^{s}\right)} .
$$

Proof. The proof is divided into several steps.

Step 1. The continuous control is smooth. Let $x^{0} \in \mathscr{D}\left(A^{s}\right)$. From Theorem 3 , the weighted HUM method yields a control $V(t)=\eta(t) B^{*} \Phi(t)$, computed by Proposition 11 where $\Phi$ is the solution of (14) corresponding to the minimizer $\Phi^{T}$ of the functional $J$ in $(23)$, which is smooth:

$$
\left\|\Phi^{T}\right\|_{\mathscr{D}\left(A^{s}\right)} \leq C\left\|x^{0}\right\|_{\mathscr{D}\left(A^{s}\right)} .
$$

Step 2. An approximate control. Since $\Phi^{T} \in \mathscr{D}\left(A^{s}\right)$, by Hypothesis \#3, one can approximate $\Phi$ by a sequence $\tilde{\Phi}_{h}$ of solutions of the discrete equations (134) with initial data $\tilde{\Phi}_{h}^{T} \in \mathfrak{C}_{h}$ such that

$$
\left\|B^{*}\left(\rho_{h} \tilde{\Phi}_{h}-\Phi\right)\right\|_{L^{2}(0, T ; \mathscr{U})} \leq C h^{\theta}\left\|\Phi^{T}\right\|_{\mathscr{D}\left(A^{s}\right)} \leq C h^{\theta}\left\|x^{0}\right\|_{\mathscr{D}\left(A^{s}\right)} .
$$

Hence, setting

$$
\tilde{\mathbf{v}}_{h}(t)=\eta(t) B_{h}^{*} \tilde{\Phi}_{h}(t)
$$

$\tilde{\mathbf{v}}_{h}$ satisfies

$$
\left\|\tilde{\mathbf{v}}_{h}-V\right\|_{L^{2}(0, T ; d t / \eta ; \mathscr{U})} \leq C h^{\theta}\left\|x^{0}\right\|_{\mathscr{D}\left(A^{s}\right)} .
$$

Then, using Hypothesis \#4, we get that the solution $\tilde{\mathbf{x}}_{h}$ of

$$
\tilde{\mathbf{x}}_{h}^{\prime}=A_{h} \tilde{\mathbf{x}}_{h}+B_{h} \tilde{\mathbf{v}}_{h}, \quad t \geq 0, \quad \tilde{\mathbf{x}}_{h}(0)=\mathbf{x}_{h}^{0},
$$

satisfies

$$
\left\|\tilde{\mathbf{x}}_{h}(T)\right\|_{h} \leq C h^{\theta}\left\|x^{0}\right\|_{\mathscr{D}\left(A^{s}\right)} .
$$

Step 3. An exact discrete control. From Theorem 14 there exists a control function $\hat{\mathbf{v}}_{h} \in L^{2}(0, T ; \mathscr{U})$ such that the function $\mathbf{w}_{h}$ solution of

$$
\mathbf{w}_{h}^{\prime}=A_{h} \mathbf{w}_{h}+B_{h} \hat{\mathbf{v}}_{h}, \quad t \geq 0, \quad \mathbf{w}_{h}(0)=0,
$$

satisfies

$$
\forall \varphi_{h}^{T} \in \mathfrak{C}_{h}, \quad\left\langle\mathbf{w}_{h}(T)+\tilde{\mathbf{x}}_{h}(T), \varphi_{h}(T)\right\rangle_{h}=0 .
$$


Besides, from Theorem 14 this can be done with a control function $\hat{\mathbf{v}}_{h} \in L^{2}(0, T ; \mathscr{U})$ that can be written $\hat{\mathbf{v}}_{h}=\eta B_{h}^{*} \zeta_{h}$ for $\zeta_{h}$ solution of (134) with initial data $\zeta_{h}^{T} \in \mathfrak{C}_{h}$, and with

$$
\left\|\hat{\mathbf{v}}_{h}\right\|_{L^{2}(0, T ; d t / \eta ; \mathscr{U})} \leq C\left\|\tilde{x}_{h}(T)\right\|_{h} \leq C h^{\theta}\left\|x^{0}\right\|_{\mathscr{D}\left(A^{s}\right)} .
$$

Hence $\tilde{\mathbf{v}}_{h}+\hat{\mathbf{v}}_{h}$ is a control for (132) (in the sense of (136)).

Step 4. Identification of the controls. From the uniqueness of the discrete controls that can be written as $\eta B_{h}^{*} \varphi_{h}$ with $\varphi_{h}^{T} \in \mathfrak{C}_{h}$ stated in Theorem 14, $\mathbf{V}_{h}=\tilde{\mathbf{v}}_{h}+\hat{\mathbf{v}}_{h}$.

Hence, from (153)-154),

$\left\|V-\mathbf{V}_{h}\right\|_{L^{2}(0, T ; d t / \eta ; \mathscr{U})} \leq\left\|V-\tilde{\mathbf{v}}_{h}\right\|_{L^{2}(0, T ; d t / \eta ; \mathscr{U})}+\left\|\hat{\mathbf{v}}_{h}\right\|_{L^{2}(0, T ; d t / \eta ; \mathscr{U})} \leq C h^{\theta}\left\|x^{0}\right\|_{\mathscr{D}\left(A^{s}\right)}$.

This completes the proof of the Theorem.

The approach presented above is very general and can be applied in many situations. Below, we shall explain how it yields convergence results from the weak observability results stated in Section 5 .

Remark 18. We refer to the recent work [20] for approximation results based on the continuous approach. In that approach the approximate controls are not built as controls for an approximate discrete dynamics but rather discretizing an iterative algorithm leading to convergence at the continuous level, but necessarily to the control of minimal norm. Note also that the method developed in [20] only converges for initial data to be controlled lying in $\mathscr{D}\left(A^{3 / 2}\right)$ (the proofs in [20] focus on the finite element methods for the wave equation, for which this space is the natural one), but does not a priori converge when the initial data to be controlled only lie in $X$. The discrete approach we develop here provides both, convergence results in the optimal class of initial data and convergence rates for smooth data.

Remark 19. In a first reading, the fact that the proof of convergence of the discrete controls does not require the convergence of the controlled equations might seem surprising. Indeed, Hypotheses \#1, \#2 and \#3 refer only to the adjoint equation (134)-(14) and only Hypothesis \#4 directly refers to the convergence of the controlled equation.

But the convergence properties of the adjoint equation (134) towards the continuous one (14) in Hypotheses \#1, \#2 and \#3 also yield convergence results for the discrete controlled system (132)-(13) since their solutions are defined by transposition, taking scalar products with the solutions of the adjoint system.

\subsection{Controllability Results}

In this Section we apply the above procedure for deriving convergence rates for numerical controls in various relevant examples.

Before going further, let us emphasize that the problem of boundary control, as the internal control problem above, corresponds to a case in which the energy space 
is not identified with its dual, as it is done in the previous paragraph. This fact creates a shift in the functional spaces below. We made the choice of presenting the abstract theory in the reflexive case with the identification between $X$ and its dual for the sake of simplicity.

More precisely, in the case of the boundary controllability of the wave equation, the adjoint equation (39) lies in $X=H_{0}^{1}(0,1) \times L^{2}(0,1)$, whereas the controlled equation (42) is solved in the space $X^{*}=L^{2}(0,1) \times H^{-1}(0,1)$.

Note in particular that the wave semigroup is an isometry in both spaces $X$ and $X^{*}$, and thus the only difference with respect to the presentation above is that the identification between $X$ and its dual is not done.

Hence, Hypotheses \#1, \#2, \#3 should be checked in the energy space $H_{0}^{1}(0,1) \times$ $L^{2}(0,1)$, whereas Hypothesis \#4, that refers to the convergence of the continuous controlled equation towards [42), should be proved in the space $L^{2}(0,1) \times$ $H^{-1}(0,1)$.

\subsubsection{Filtering methods}

Based on Theorem 10, we can set $\mathfrak{C}_{h}=\mathscr{C}_{h}\left(\gamma / h^{2}\right)$ with $\gamma \in(0,4)$. Note that, here $\mathscr{C}_{h}\left(\gamma / h^{2}\right)$ refers to the space in which the trajectories $\mathbf{u}_{h}$, solutions of 81b, live. Of course, this can be identified with the set of data such that for some $t \in(0, T)$ (and then for all $t \in(0, T)),\left(\mathbf{u}_{h}(t), \mathbf{u}_{h}^{\prime}(t)\right)$ belongs to the vector space spanned by the first eigenvectors $\mathbf{w}_{h}^{k}$ corresponding to the eigenvalues $\lambda_{h}^{k} \leq \gamma / h^{2}$.

In that case, the control requirement (136) for solutions $\mathbf{y}_{h}$ of (98) becomes:

$$
\forall \mathbf{u}_{h} \in \mathscr{C}_{h}\left(\gamma / h^{2}\right), \quad h \sum_{j=1}^{N} y_{j}(T) u_{j}^{\prime}(T)-h \sum_{j=1}^{N} y_{j}^{\prime}(T) u_{j}(T)=0
$$

or, equivalently,

$$
\pi_{\mathscr{C}_{h}\left(\gamma / h^{2}\right)} \mathbf{y}_{h}(T)=0, \text { and } \pi_{\mathscr{C}_{h}\left(\gamma / h^{2}\right)} \mathbf{y}_{h}^{\prime}(T)=0,
$$

where $\pi_{\mathscr{C}_{h}\left(\gamma / h^{2}\right)}$ denotes the orthogonal projection of $L_{h}^{2}(0,1)$ on the vector space spanned by the eigenfunctions $\mathbf{w}_{h}^{k}$ corresponding to eigenvalues $\lambda_{h}^{k} \leq \gamma / h^{2}$.

Fix now $\gamma \in(0,4)$, and $T>T(\gamma)$ given by Theorem 10. Introduce $\delta>0$ such that $T>T(\gamma)+2 \delta$. Let $\eta$ be a smooth function of time such that

$$
\eta: \mathbb{R} \rightarrow[0,1], \quad \eta(t)=\left\{\begin{array}{l}
1 \text { on }[\delta, T(\gamma)+\delta] \\
0 \text { on } \mathbb{R} \backslash(0, T) .
\end{array}\right.
$$

According to the analysis done in the previous Section, it is then natural to introduce the following functional

$$
J_{h}\left(\mathbf{u}_{h}\right)=\frac{1}{2} \int_{0}^{T} \eta(t)\left|\frac{u_{N}(t)}{h}\right|^{2} d t+h \sum_{j=1}^{N} y_{j}^{0} u_{j}^{1}-h \sum_{j=1}^{N} y_{j}^{1} u_{j}^{0},
$$


for $\mathbf{u}_{h} \in \mathscr{C}_{h}\left(\gamma / h^{2}\right)$.

Then, similarly as in Theorem 7 we have:

Theorem 17. Let $\gamma \in(0,4)$ and $T>T(\gamma)$ given by Theorem 10 .

For all $h>0$ system (98) is controllable in the sense of 155) (or, equivalently, (156)).

More precisely, for any $\left(\mathbf{y}_{h}^{0}, \mathbf{y}_{h}^{1}\right) \in \mathbb{R}^{N} \times \mathbb{R}^{N}$, there exists a control $\mathbf{V}_{h} \in L^{2}(0, T ; d t / \eta)$ such that the solution of (98) satisfies (155).

Moreover, the control $\mathbf{V}_{h}$ of minimal $L^{2}(0, T ; d t / \eta)$-norm fulfilling (155) can be characterized through the minimization (over $\mathscr{C}_{h}\left(\gamma / h^{2}\right)$ ) of the functional $J_{h}$ in (158) as

$$
\mathbf{V}_{h}(t)=-\eta(t) \frac{U_{N}(t)}{h},
$$

where $\mathbf{U}_{h}$ is the minimizer of $J_{h}$ in (158) over $\mathscr{C}_{h}\left(\gamma / h^{2}\right)$.

Here, the difference with the situation in Theorem 7 is that discrete systems are observable within the space $\mathscr{C}_{h}\left(\gamma / h^{2}\right)$, uniformly with respect to the discretization parameter $h>0$. This allows to deduce that the discrete controls $\mathbf{V}_{h}$ given by Theorem 17 are bounded.

One should then prove that the Hypotheses \#1 and \#2 hold in this case, to obtain a convergence result. In this case, they take the following form:

Lemma $2\left([\mathbf{5 3},[\mathbf{3 6}])\right.$. Let $\left(u^{0}, u^{1}\right) \in C_{0}^{\infty}(0,1)^{2}$ and $u$ be the corresponding solution of 39. Then there exists a sequence of functions $\mathbf{u}_{h} \in \mathscr{C}_{h}\left(\gamma / h^{2}\right)$ such that

$$
\begin{gathered}
\left(\rho_{h} \mathbf{u}_{h}(0), \rho_{h} \mathbf{u}_{h}^{\prime}(0)\right) \underset{h \rightarrow 0}{\longrightarrow}\left(u(0), u^{\prime}(0)\right) \quad H_{0}^{1}(0,1) \times L^{2}(0,1) \\
-\frac{u_{N, h}}{h} \underset{h \rightarrow 0}{\longrightarrow} \partial_{x} u(1, t) \quad L^{2}(0, T)
\end{gathered}
$$

where $\rho_{h}$ is the continuous extension of the discrete function $\mathbf{u}_{h}$ by Fourier series.

In other words, Hypothesis \#1 is satisfied in this case. Corresponding to Hypothesis \#2, we have:

Lemma $3\left([\mathbf{5 3},[\mathbf{3 6}])\right.$. Let $\left(\mathbf{u}_{h}^{0}, \mathbf{u}_{h}^{1}\right)$ be discrete functions and $\left(u^{0}, u^{1}\right) \in H_{0}^{1}(0,1) \times$ $L^{2}(0,1)$ such that

$$
\left(\rho_{h} \mathbf{u}_{h}^{0}, \rho_{h} \mathbf{u}_{h}^{1}\right) \underset{h \rightarrow 0}{\rightarrow}\left(u^{0}, u^{1}\right) \quad H_{0}^{1}(0,1) \times L^{2}(0,1)
$$

and

$$
\sup _{h}\left\|\frac{u_{N, h}(t)}{h}\right\|_{L^{2}(0, T)}<\infty .
$$

Then, denoting by $\mathbf{u}_{h}$ and $u$ respectively the solutions of (81) and 39) with initial data $\left(\mathbf{u}_{h}^{0}, \mathbf{u}_{h}^{1}\right)$ and $\left(u^{0}, u^{1}\right)$ respectively, we have 


$$
\begin{gathered}
\left(\rho_{h} \mathbf{u}_{h}, \rho_{h} \mathbf{u}_{h}^{\prime}\right) \underset{h \rightarrow 0}{\rightarrow}\left(u, u^{\prime}\right) \quad L^{2}\left(0, T ; H_{0}^{1}(0,1) \times L^{2}(0,1)\right) \\
-\frac{u_{N, h}}{h} \underset{h \rightarrow 0}{\rightarrow} \partial_{x} u(1, t) \quad L^{2}(0, T) \\
\left(\rho_{h} \mathbf{u}_{h}^{0}, \rho_{h} \mathbf{u}_{h}^{1}\right) \underset{h \rightarrow 0}{\rightarrow}\left(u^{0}, u^{1}\right) \quad H_{0}^{1}(0,1) \times L^{2}(0,1) .
\end{gathered}
$$

Here, again, $\rho_{h}$ denotes the continuous extension operator of discrete functions by Fourier series.

In other words, Hypothesis \#2 is satisfied in this case.

Note that, due to the multiplier identity $(110)$, one easily checks that 163 ) is a consequence of (162). Indeed, weakly convergent sequences are bounded, and (110) immediately yields an uniform admissibility result for the discrete wave equation 81).

We refer to [53, 36] for the proof of Lemmas 2,3

Accordingly, based on the convergence result in Theorem 15, we get

Theorem 18. Within the setting of Theorem 17 given $\left(y^{0}, y^{1}\right) \in L^{2}(0,1) \times H^{-1}(0,1)$ and a sequence of discrete initial data $\left(\mathbf{y}_{h}^{0}, \mathbf{y}_{h}^{1}\right)$ such that $\left(\rho_{h} \mathbf{y}_{h}^{0}, \rho_{h} \mathbf{y}_{h}^{1}\right)$ weakly converges to $\left(y^{0}, y^{1}\right)$ in $L^{2}(0,1) \times H^{-1}(0,1)$, the discrete controls $\mathbf{V}_{h}$ provided by Theorem 17 weakly converges in $L^{2}(0, T ; d t / \eta)$ to $V$, the control provided by Theorem [5] as $h \rightarrow 0$.

Besides, if the discrete initial data $\left(\mathbf{y}_{h}^{0}, \mathbf{y}_{h}^{1}\right)$ are such that $\left(\rho_{h} \mathbf{y}_{h}^{0}, \rho_{h} \mathbf{y}_{h}^{1}\right)$ strongly converges to $\left(y^{0}, y^{1}\right)$ in $L^{2}(0,1) \times H^{-1}(0,1)$, then the discrete controls $\mathbf{V}_{h}$ strongly converges to $V$ in $L^{2}(0, T ; d t / \eta)$ as $h \rightarrow 0$.

It is then natural to address the issue of the convergence rates for the discrete controls $\mathbf{V}_{h}$ given by Theorem 17. For this to be done, as we have said, it is sufficient to derive the order of convergence for the discrete wave equation, namely the ones corresponding to Hypotheses \#3 and \#4.

The following result is proved in [36]:

Proposition 4 ([36]). Let $\left(u^{0}, u^{1}\right) \in H^{2} \cap H_{0}^{1}(0,1) \times H_{0}^{1}(0,1)$. Then there exists a constant $C=C(T)$ independent of $\left(u^{0}, u^{1}\right)$ and a sequence $\left(\mathbf{u}_{h}^{0}, \mathbf{u}_{h}^{1}\right) \in \mathscr{C}_{h}\left(1 / h^{4 / 3}\right)$ of initial data such that for all $h>0$,

$$
\left\|\left(\rho_{h} \mathbf{u}_{h}^{0}, \rho_{h} \mathbf{u}_{h}^{1}\right)-\left(u^{0}, u^{1}\right)\right\|_{H_{0}^{1} \times L^{2}} \leq C h^{2 / 3}\left\|\left(u^{0}, u^{1}\right)\right\|_{H^{2} \cap H_{0}^{1} \times H_{0}^{1}}
$$

and the solutions $u$ of (39) with initial data $\left(u^{0}, u^{1}\right)$ and $\mathbf{u}_{h}$ of $(81$ with initial data $\left(\mathbf{u}_{h}^{0}, \mathbf{u}_{h}^{1}\right)$ satisfy, for all $h>0$,

$$
\begin{gathered}
\sup _{t \in[0, T]}\left\|\left(\rho_{h} \mathbf{u}_{h}(t), \rho_{h} \mathbf{u}_{h}^{\prime}(t)\right)-\left(u(t), u^{\prime}(t)\right)\right\|_{H_{0}^{1} \times L^{2}} \leq C h^{2 / 3}\left\|\left(u^{0}, u^{1}\right)\right\|_{H^{2} \cap H_{0}^{1} \times H_{0}^{1}}, \\
\left\|\frac{u_{N, h}(\cdot)}{h}+u_{x}(1, \cdot)\right\|_{L^{2}(0, T)} \leq C h^{2 / 3}\left\|\left(u^{0}, u^{1}\right)\right\|_{H^{2} \cap H_{0}^{1} \times H_{0}^{1}}, \quad h>0 .
\end{gathered}
$$

Moreover, 


$$
\begin{gathered}
\sup _{t \in[0, T]}\left\|\left(\rho_{h} \mathbf{u}_{h}(t), \rho_{h} \mathbf{u}_{h}^{\prime}(t)\right)\right\|_{H^{2} \cap H_{0}^{1} \times H_{0}^{1}} \leq C\left\|\left(u^{0}, u^{1}\right)\right\|_{H^{2} \cap H_{0}^{1} \times H_{0}^{1}}, \\
\left\|\frac{u_{N, h}(\cdot)}{h}\right\|_{H^{1}(0, T)} \leq C\left\|\left(u^{0}, u^{1}\right)\right\|_{H^{2} \cap H_{0}^{1} \times H_{0}^{1}}, \quad h>0 .
\end{gathered}
$$

Note that Proposition 4 is proved by taking the Fourier series decomposition of the continuous solution $u$ of 39 and truncating it at the best order, which turns out to be $\lambda_{h}^{k} \simeq 1 / h^{4 / 3}$. This might be surprising since it introduces powers of the form $h^{2 / 3}$ for the rate of convergence of the numerical scheme. But, actually, this strategy is optimal, as explained in [91]. This is due to the fact that

$$
\sqrt{\lambda_{h}^{k}}-k \pi=\frac{2}{h} \sin \left(\frac{k \pi h}{2}\right)-k \pi \simeq \frac{\pi^{3}}{24} k^{3} h^{2},
$$

which is small within the range of $k$ such that $k \lesssim h^{-2 / 3}$, hence corresponding to $\lambda_{h}^{k} \lesssim h^{-4 / 3}$.

Also note that $\rho_{h}$ denotes the Fourier extension of the discrete solutions. Hence it is smooth and one can take the $H^{2}(0,1)$ norms of these continuous approximations as required in the statement above.

Finally, let us emphasize that Proposition 4 is well-known except for what concerns the convergence of the normal derivatives on the boundary. In particular, our approach strongly uses the uniform hidden regularity property given by the multiplier identity (110).

Once this is done, we are in position to state the following counterpart of Hypothesis \#4:

Theorem 19 ([36]). Let $\left(y^{0}, y^{1}\right) \in H_{0}^{1}(0,1) \times L^{2}(0,1)$ and $v \in H_{0}^{1}(0, T)$ and denote by $y$ the corresponding solution of 42.

Consider a sequence of initial data $\left(\mathbf{y}_{h}^{0}, \mathbf{y}_{h}^{1}\right)$ and control functions $\mathbf{v}_{h} \in L^{2}(0, T)$ such that

$$
\sup _{h>0}\left\{\left\|\left(\rho_{h} \mathbf{y}_{h}^{0}, \rho_{h} \mathbf{y}_{h}^{1}\right)\right\|_{H_{0}^{1} \times L^{2}}+\left\|\mathbf{v}_{h}\right\|_{H_{0}^{1}(0, T)}\right\}<\infty
$$

and denote by $\mathbf{y}_{h}$ the corresponding solution of (98). Then there exists a positive constant $C$ independent of $h>0$ such that

$$
\begin{aligned}
& \left\|\left(\rho_{h} y_{h}(T), \rho_{h} y_{h}^{\prime}(T)\right)-\left(y(T), y^{\prime}(T)\right)\right\|_{L^{2} \times H^{-1}} \\
& \leq C h^{2 / 3} \sup _{h>0}\left\{\left\|\left(\rho_{h} \mathbf{y}_{h}^{0}, \rho_{h} \mathbf{y}_{h}^{1}\right)\right\|_{H_{0}^{1} \times L^{2}}+\left\|\mathbf{v}_{h}\right\|_{H_{0}^{1}(0, T)}\right\} \\
& \quad+\left\|\left(\rho_{h} \mathbf{y}_{h}^{0}, \rho_{h} \mathbf{y}_{h}^{1}\right)-\left(y^{0}, y^{1}\right)\right\|_{L^{2} \times H^{-1}}+C\left\|\mathbf{v}_{h}-v\right\|_{L^{2}(0, T)} .
\end{aligned}
$$

The details of the proof of Theorem 19 will be given in [36].

This is slightly more subtle than Proposition 4 at least for two reasons:

- To give a precise definition of the solution of the wave equation with initial data in $L^{2}(0,1) \times H^{-1}(0,1)$ with a boundary data $v \in L^{2}(0, T)$, one needs to introduce 
the concept of solutions in the sense of transposition, i.e. based on the duality with solutions $u$ of equations similar to 39 lying in the energy space $H_{0}^{1}(0,1) \times$ $L^{2}(0,1)$, and to use hidden regularity results that show that $u_{x}(1, t) \in L^{2}(0, T)$, see [68].

- One should then use the explicit convergence results stated in Proposition4 and in particular the one on the normal derivative 169.

Then, using Proposition 4 and Theorem 19 and Theorem 16 we get:

Theorem 20. Let $\left(y^{0}, y^{1}\right) \in H_{0}^{1}(0,1) \times L^{2}(0,1)$ and consider a sequence of discrete initial data $\left(\mathbf{y}_{h}^{0}, \mathbf{y}_{h}^{1}\right)$ such that $\left(\rho_{h} \mathbf{y}_{h}^{0}, \rho_{h} \mathbf{y}_{h}^{1}\right)$ strongly converges to $\left(y^{0}, y^{1}\right)$ in $L^{2}(0,1) \times$ $H^{-1}(0,1)$.

Let $\gamma \in(0,4)$ and $T>T(\gamma)$. Then the controls $\mathbf{V}_{h}$ given by Theorem [17 strongly converge to $V$ in $L^{2}(0, T ; d t / \eta)$, where $V$ is the control given by Theorem $[$ corresponding to $\left(y^{0}, y^{1}\right)$.

Besides, there exists a constant $C$ such that for all $h>0$,

$$
\begin{aligned}
\left\|\mathbf{V}_{h}-V\right\|_{L^{2}(0, T ; d t / \eta)} \leq C h^{2 / 3} \sup _{h>0}\{ & \left.\left\|\left(\rho_{h} \mathbf{y}_{h}^{0}, \rho_{h} \mathbf{y}_{h}^{1}\right)\right\|_{H_{0}^{1} \times L^{2}}\right\} \\
& +C\left\|\left(\rho_{h} \mathbf{y}_{h}^{0}, \rho_{h} \mathbf{y}_{h}^{1}\right)-\left(y^{0}, y^{1}\right)\right\|_{L^{2} \times H^{-1}} .
\end{aligned}
$$

In particular, choosing $\left(\mathbf{y}_{h}^{0}, \mathbf{y}_{h}^{1}\right)$ such that for some $C$ independent of $h>0$,

$$
\begin{gathered}
\left\|\left(\rho_{h} \mathbf{y}_{h}^{0}, \rho_{h} \mathbf{y}_{h}^{1}\right)\right\|_{H_{0}^{1} \times L^{2}} \leq C\left\|\left(y^{0}, y^{1}\right)\right\|_{H_{0}^{1} \times L^{2}}, \\
\left\|\left(\rho_{h} \mathbf{y}_{h}^{0}, \rho_{h} \mathbf{y}_{h}^{1}\right)-\left(y^{0}, y^{1}\right)\right\|_{L^{2} \times H^{-1}} \leq C h^{2 / 3}\left\|\left(y^{0}, y^{1}\right)\right\|_{H_{0}^{1} \times L^{2}},
\end{gathered}
$$

one immediately gets

$$
\left\|\mathbf{V}_{h}-V\right\|_{L^{2}(0, T ; d t / \eta)} \leq C h^{2 / 3}\left\|\left(y^{0}, y^{1}\right)\right\|_{H_{0}^{1} \times L^{2}} .
$$

To our knowledge, this is the first result on the order of convergence for the discrete controls obtained in Theorem 17

Let us also emphasize that the convergence results stated in (174)-(175) are satisfied when taking as discrete initial data the restriction to the mesh points of the orthogonal projections in $L^{2}(0,1)$ or $H_{0}^{1}(0,1)$ on the vector space spanned by the functions $\left(w^{k}(x)=\sin (k \pi x)\right)_{1 \leq k \leq N}$. Of course, other interpolation operators can be considered for which assumptions (174)-(175) are satisfied.

Remark 20. The observability results in classes of filtered solutions stated in Section 5.4 and obtained in [111] for the semidiscrete finite-difference approximations of the multi-dimensional wave equation, also yield similar convergence estimates with proofs that follow line to line those above. We do not write down the details here for the sake of conciseness.

The results stated in Theorem 13 ([27]) do not apply in the context of boundary controllability, but rather when the control is distributed inside the domain. In that case one does not need to use transposition methods since solutions are defined in a 
classical manner and this can be done by standard energy and semigroup methods (see Theorem 4). Consequently, the needed convergence results are more classical. But still, to our knowledge, a rigorous proof of the fact that Hypothesis \#3 holds in that case is still missing.

Of course, despite of this, Hypotheses \#1 and \#2 hold and follow from classical convergence results for the finite element methods, see [4]. Therefore, one can prove the counterpart of Theorem 18] in that case, see [27] for details.

\subsubsection{The bi-grid technique}

The methods above can also be used to obtain convergence results and convergence rates for the two-grid filtering technique.

In this case $\mathfrak{C}_{h}=\mathscr{V}_{h}$, where $\mathscr{V}_{h}$ is given by (113). We are then precisely in the same setting as the one in Section 6.1

Based on the observability result stated in Theorem 11, using Theorem 14 we obtain:

Theorem 21. Let $T>2 \sqrt{2}$ and $\eta$ be as in (157) with $T(\gamma)$ replaced by $2 \sqrt{2}$.

Let $\left(\mathbf{y}_{h}^{0}, \mathbf{y}_{h}^{1}\right)$ be discrete initial data.

Then introduce the functional $J_{h}$ defined as in (158) for $\mathbf{u}_{h}$ solution of (81) such that $\left(\mathbf{u}_{h}(T), \mathbf{u}_{h}^{\prime}(T)\right) \in \mathscr{V}_{h}$. This functional has a unique minimizer $\mathbf{U}_{h}$ solution of (81) with $\left(\mathbf{U}_{h}(T), \mathbf{U}_{h}^{\prime}(T)\right) \in \mathscr{V} / h$, among the space of solutions $\mathbf{u}_{h}$ such that $\left(\mathbf{u}_{h}(T), \mathbf{u}_{h}^{\prime}(T)\right) \in \mathscr{V}_{h}$.

Then $\mathbf{V}_{h}$ defined as in (159) is a control function for which the solution $\mathbf{y}_{h}$ of (98) satisfies

$$
\forall\left(\mathbf{u}_{h}^{0, T}, \mathbf{u}_{h}^{1, T}\right) \in \mathscr{V}_{h}, \quad h \sum_{j=1}^{N} y_{j}(T) u_{j}^{1, T}-h \sum_{j=1}^{N} y_{j}^{\prime}(T) u_{j}^{0, T}=0 .
$$

Moreover, $\mathbf{V}_{h}$ is the control of minimal $L^{2}(0, T ; d t / \eta)$ norm for which the corresponding solution of (98) satisfies the control requirement 1777. It is also the only control satisfying (177) that can be written as in (159) for a solution $\mathbf{u}_{h}$ of (81) with $\left(\mathbf{u}_{h}(T), \mathbf{u}_{h}^{\prime}(T)\right) \in \mathscr{V}_{h}$.

Now, using Theorem 15, Lemma 3 and an easy variant of Lemma 2 left to the reader, one can then prove the following:

Theorem 22 ([36]). Within the setting of Theorem 21] given $\left(y^{0}, y^{1}\right) \in L^{2}(0,1) \times$ $H^{-1}(0,1)$ and a sequence of discrete initial data $\left(\mathbf{y}_{h}^{0}, \mathbf{y}_{h}^{1}\right)$ such that $\left(\rho_{h} \mathbf{y}_{h}^{0}, \rho_{h} \mathbf{y}_{h}^{1}\right)$ weakly converges to $\left(y^{0}, y^{1}\right)$ in $L^{2}(0,1) \times H^{-1}(0,1)$, the discrete controls $\mathbf{V}_{h}$ provided by Theorem 21 weakly converge in $L^{2}(0, T ; d t / \eta)$ to $V$, the control provided by Theorem [5] as $h \rightarrow 0$.

Besides, if the discrete initial data $\left(\mathbf{y}_{h}^{0}, \mathbf{y}_{h}^{1}\right)$ are such that $\left(\rho_{h} \mathbf{y}_{h}^{0}, \rho_{h} \mathbf{y}_{h}^{1}\right)$ strongly converge to $\left(y^{0}, y^{1}\right)$ in $L^{2}(0,1) \times H^{-1}(0,1)$, the discrete controls $\mathbf{V}_{h}$ strongly converge to $V$ in $L^{2}(0, T ; d t / \eta)$ as $h \rightarrow 0$. 
To go further, one should then prove a variant of Proposition 4 for the solutions $\mathbf{u}_{h}$ of the discrete wave equations (81) such that $\left(\mathbf{u}_{h}(T), \mathbf{u}_{h}^{\prime}(T)\right) \in \mathscr{V}_{h}$. One way of doing that is to take the discrete solutions given by (4), which belong to $\mathscr{C}_{h}\left(1 / h^{4 / 3}\right)$ and to add to them high-frequency components so that $\left(\mathbf{u}_{h}(T), \mathbf{u}_{h}^{\prime}(T)\right) \in \mathscr{V}_{h}$. Doing this, one can check that the high-frequency components that have been added that way are small and do not modify the estimates in Proposition 4.

Note that, of course, these approximations will not belong anymore to $\mathscr{C}_{h}\left(1 / h^{4 / 3}\right)$ but it does not matter for our purpose.

Then, using Theorem 15 , Theorem 19 and this slightly modified variant of Proposition 4 where we further imposed on the discrete data the condition $\left(\mathbf{u}_{h}(T), \mathbf{u}_{h}^{\prime}(T)\right) \in$ $\mathscr{V}_{h}$, one can obtain convergence rates for the convergence of the discrete controls:

Theorem 23 ([36]). Let $\left(y^{0}, y^{1}\right) \in H_{0}^{1}(0,1) \times L^{2}(0,1)$ and consider a sequence of discrete initial data $\left(\mathbf{y}_{h}^{0}, \mathbf{y}_{h}^{1}\right)$ such that $\left(\rho_{h} \mathbf{y}_{h}^{0}, \rho_{h} \mathbf{y}_{h}^{1}\right)$ strongly converges to $\left(y^{0}, y^{1}\right)$ in $L^{2}(0,1) \times H^{-1}(0,1)$.

Let $T>2 \sqrt{2}$. Then the controls $\mathbf{V}_{h}$ given by Theorem 21 strongly converge to $V$ in $L^{2}(0, T ; d t / \eta)$, where $V$ is the control given by Theorem 5 corresponding to $\left(y^{0}, y^{1}\right)$.

Besides, there exists a constant $C$ such that for all $h>0$, estimate (173) holds. In particular, choosing $\left(\mathbf{y}_{h}^{0}, \mathbf{y}_{h}^{1}\right)$ such that for some $C$ independent of $h>0$, (174)-175 are satisfied, one immediately gets 176.).

The proof can be found in [36] but, again, it follows the general theory developed in Section 6.1.

\subsubsection{Tychonoff regularization}

The Tychonoff regularization is of slightly different nature since, in agreement with Theorem 12, one has to reinforce the observation operator by adding an extra observation, distributed everywhere in the discrete grid, so that observability holds uniformly on the mesh-size parameter for all solutions. In view of this, the applied control mechanism has to be reinforced as well, so that to the boundary discrete control we will add an extra control distributed everywhere in the domain. However, this added control will vanish as $h \rightarrow 0$ and the methods of Section 6.1 will apply to show the convergence towards the limit control of the leading term. There are however some minor modifications to be introduced with respect to the abstract functional setting provided in Section 6.1 that we describe below.

Let $\eta$ be as in (157) with $T(\gamma)$ replaced by 2 .

First, we introduce the functional $\hat{J}_{h}$ defined for $\left(\mathbf{u}_{h}^{0}, \mathbf{u}_{h}^{1}\right) \in \mathbb{R}^{N} \times \mathbb{R}^{N}$ by: 


$$
\begin{array}{r}
\hat{J}_{h}\left(\mathbf{u}_{h}^{0}, \mathbf{u}_{h}^{1}\right)=\frac{1}{2} \int_{0}^{T} \eta(t)\left|\frac{u_{N}(t)}{h}\right|^{2} d t+\frac{h^{3}}{4} \sum_{j=0}^{N} \int_{0}^{T} \eta(t)\left|\frac{u_{j+1}^{\prime}-u_{j}^{\prime}}{h}\right|^{2} d t \\
+h \sum_{j=1}^{N} y_{j}^{0} u_{j}^{1}-h \sum_{j=1}^{N} y_{j}^{1} u_{j}^{0}
\end{array}
$$

where $\mathbf{u}_{h}$ is the solution of the adjoint system 81 with initial datum $\left(\mathbf{u}_{h}^{0}, \mathbf{u}_{h}^{1}\right)$.

Using this functional and based on Theorem 12, we get the following:

Theorem 24. Set $T>2$, and consider an initial datum $\left(\mathbf{y}_{h}^{0}, \mathbf{y}_{h}^{1}\right) \in \mathbb{R}^{N} \times \mathbb{R}^{N}$.

For each $h>0$, the functional $\hat{J}_{h}$ in 178 has a unique minimizer $\left(\mathbf{U}_{h}^{0}, \mathbf{U}_{h}^{1}\right)$. Then, setting

$$
\left\{\begin{array}{l}
\mathbf{V}_{h}(t)=-\eta(t) \frac{U_{N}(t)}{h} \\
G_{j, h}(t)=\frac{\eta(t)}{2 h^{2}}\left(U_{j+1}^{\prime}-2 U_{j}^{\prime}+U_{j-1}^{\prime}\right), \quad j=1, \cdots, N,
\end{array}\right.
$$

the solution $\mathbf{y}_{h}$ of

$$
\begin{cases}y_{j}^{\prime \prime}-\frac{1}{h^{2}}\left[y_{j+1}+y_{j-1}-2 y_{j}\right]=h^{2} G_{j, h}^{\prime}, & 0<t<T, j=1, \ldots, N \\ y_{0}(t)=0 ; y_{N+1}(t)=\mathbf{V}_{h}(t), & 0<t<T \\ y_{j}(0)=y_{j}^{0}, y_{j}^{\prime}(0)=y_{j}^{1}, & j=1, \ldots, N,\end{cases}
$$

satisfies the control requirement

$$
\left(\mathbf{y}_{h}(T), \mathbf{y}_{h}^{\prime}(T)\right)=(0,0) .
$$

Theorem 24 shows how the Tychonoff regularization modifies the control problem. It introduces a control everywhere in the domain, that weakly converges to zero. This is of course compatible with our analysis, which states the existence of high-frequency spurious solutions which do not propagate and therefore can not be controlled from the boundary. Therefore, if one wants to satisfy the strong control requirement 181, one needs to introduce a control everywhere in the domain. But this control can be built in such a way that it vanishes when $h \rightarrow 0$.

Note that Theorem 12 gives a lot more of information, and in particular the following one:

Proposition 5. Under the assumptions of Theorem 24 there exists a constant $C(T)$ independent of $h>0$ such that

$$
\begin{array}{r}
\left\|\mathbf{V}_{h}\right\|_{L^{2}(0, T)}+h\left\|\rho_{h} \mathbf{G}_{h}\right\|_{L^{2}\left(0, T ; H^{-1}(0,1)\right)}+h^{2}\left\|\rho_{h} \mathbf{G}_{h}\right\|_{L^{\infty}\left(0, T ; L^{2}(0,1)\right)} \\
\leq C(T)\left\|\left(\rho_{h} \mathbf{y}_{h}^{0}, \rho_{h} \mathbf{y}_{h}^{1}\right)\right\|_{L^{2}(0,1) \times H^{-1}(0,1)}
\end{array}
$$

We now state the following counterparts of Lemma 2 and 3 :

Lemma 4 ([36]). In the setting of Lemma 2 (with $\gamma=4$ so that no filtering is implemented), we further have 


$$
h^{2} \rho_{h} \Delta_{h} \mathbf{u}_{h}^{\prime} \underset{h \rightarrow 0}{\longrightarrow} 0 \quad L^{2}((0, T) \times(0,1)) .
$$

Lemma 5 ([36]). In the setting of Lemma 3$]$ we further have

$$
h^{2} \rho_{h} \Delta_{h} \mathbf{u}_{h}^{\prime} \underset{h \rightarrow 0}{\rightarrow 0} \quad L^{2}((0, T) \times(0,1)) .
$$

Based on Proposition 5 Lemmas 45 and using the same ideas as in Theorem 15 , one gets the following:

Theorem 25 ([36]). Within the setting of Theorem 24 given $\left(y^{0}, y^{1}\right) \in L^{2}(0,1) \times$ $H^{-1}(0,1)$ and a sequence of discrete initial data $\left(\mathbf{y}_{h}^{0}, \mathbf{y}_{h}^{1}\right)$ such that $\left(\rho_{h} \mathbf{y}_{h}^{0}, \rho_{h} \mathbf{y}_{h}^{1}\right)$ weakly converges to $\left(y^{0}, y^{1}\right)$ in $L^{2}(0,1) \times H^{-1}(0,1)$, then the discrete controls $\left(\mathbf{V}_{h}, \mathbf{G}_{h}\right)$ provided by Theorem 24 weakly converge in the following sense:

$$
\begin{array}{ll}
\mathbf{V}_{h} \underset{h \rightarrow 0}{\rightarrow} V, & \text { in } L^{2}(0, T ; d t / \eta), \\
h^{2} \rho_{h} \mathbf{G}_{h} \underset{h \rightarrow 0}{\rightarrow} 0, & \text { in } L^{2}((0, T) \times(0,1)),
\end{array}
$$

where $V$ is the control provided by Theorem $[5$ as $h \rightarrow 0$.

Besides, if the discrete initial data $\left(\mathbf{y}_{h}^{0}, \mathbf{y}_{h}^{1}\right)$ are such that $\left(\rho_{h} \mathbf{y}_{h}^{0}, \rho_{h} \mathbf{y}_{h}^{1}\right)$ strongly converges to $\left(y^{0}, y^{1}\right)$ in $L^{2}(0,1) \times H^{-1}(0,1)$, then the discrete controls $\left(\mathbf{V}_{h}, h^{2} \rho_{h} \mathbf{G}_{h}\right)$ strongly converge to $(V, 0)$ in $L^{2}(0, T ; d t / \eta) \times L^{2}((0, T) \times(0,1))$ as $h \rightarrow 0$.

One can even follow the proof of Theorem 16 to obtain convergence rates for the discrete controls. For doing that, inspecting the proof of Theorem 19 , we need the following for the convergence of the equations of (81) to 39):

Proposition 6 ([36]). In the setting of Lemma 3 we further have

$$
\sup _{t}\left\|h \rho_{h} \Delta_{h} \mathbf{u}_{h}^{\prime}(t)\right\|_{L^{2}(0,1)} \leq C h\left\|\left(u^{0}, u^{1}\right)\right\|_{H^{2} \cap H_{0}^{1} \times H_{0}^{1}} .
$$

The proof of this additional estimate is easy: Basically, it uses that $h \nabla_{h}$ are uniformly bounded with norm smaller than 2 , and then

$$
\left\|h \rho_{h} \Delta_{h} \mathbf{u}_{h}^{\prime}\right\|_{L^{2}(0,1)} \lesssim\left\|\rho_{h} \mathbf{u}_{h}^{\prime}\right\|_{H_{0}^{1}} \lesssim\left\|\left(\rho_{h} \mathbf{u}_{h}^{0}, \rho_{h} \mathbf{u}_{h}^{1}\right)\right\|_{H^{2} \cap H_{0}^{1} \times H_{0}^{1}} .
$$

We also need to be able to give an estimate on the controlled equation, which is mainly the one in Theorem 19 except that an internal control in $H^{-1}\left(0, T ; L^{2}(0,1)\right)$ has been added. When the distributed source terms are in $L^{2}\left(0, T ; L^{2}(0,1)\right)$ convergence results in the energy space are classical and can be found, for instance, in [4]. One can easily deal with source terms in $H^{-1}\left(0, T ; L^{2}(0,1)\right)$ integrating the equations in time, and working in the space $L^{2}(0,1) \times H^{-1}(0,1)$.

Hence we can derive the following result:

Theorem 26 ([36]). Within the setting of Theorem 25$]$ let $\left(y^{0}, y^{1}\right) \in H_{0}^{1}(0,1) \times$ $L^{2}(0,1)$ and consider a sequence of discrete initial data $\left(\mathbf{y}_{h}^{0}, \mathbf{y}_{h}^{1}\right)$ such that $\left(\rho_{h} \mathbf{y}_{h}^{0}, \rho_{h} \mathbf{y}_{h}^{1}\right)$ strongly converge to $\left(y^{0}, y^{1}\right)$ in $L^{2}(0,1) \times H^{-1}(0,1)$. 


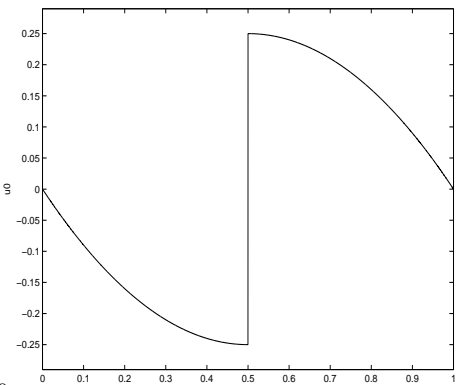

Fig. 11 The initial position $y^{0}$ to be controlled.

Let $T>2$. Then the controls $\left(\mathbf{V}_{h}, h^{2} \mathbf{G}_{h}\right)$ given by Theorem 24 strongly converge to $(V, 0)$ in $L^{2}(0, T ; d t / \eta) \times L^{2}((0, T) \times(0,1))$, where $V$ is the control given by Theorem 5 corresponding to $\left(y^{0}, y^{1}\right)$.

Besides, there exists a constant $C$ such that for all $h>0$,

$$
\begin{aligned}
& \left\|\mathbf{V}_{h}-V\right\|_{L^{2}(0, T ; d t / \eta)}+\left\|h^{2} \rho_{h} \mathbf{G}_{h}\right\|_{L^{2}((0, T) \times(0,1))} \\
& \leq C h^{2 / 3} \sup _{h>0}\left\{\left\|\left(\rho_{h} \mathbf{y}_{h}^{0}, \rho_{h} \mathbf{y}_{h}^{1}\right)\right\|_{H_{0}^{1} \times L^{2}}\right\}+\left\|\left(\rho_{h} \mathbf{y}_{h}^{0}, \rho_{h} \mathbf{y}_{h}^{1}\right)-\left(y^{0}, y^{1}\right)\right\|_{L^{2} \times H^{-1}} .
\end{aligned}
$$

In particular, if 174-175) are satisfied, we get

$$
\left\|\mathbf{V}_{h}-V\right\|_{L^{2}(0, T ; d t / \eta)}+\left\|h^{2} \rho_{h} \mathbf{G}_{h}\right\|_{L^{2}((0, T) \times(0,1))} \leq C h^{2 / 3}\left\|\left(y^{0}, y^{1}\right)\right\|_{H_{0}^{1} \times L^{2}} .
$$

The precise proofs will be given in [36], but here again, they rely on the same ideas as for Theorem 16 . Indeed it consists in using that the minimizer $\left(U^{0}, U^{1}\right)$ of the continuous HUM functional is smooth. Therefore, one can approximate it with a known error term by a discrete solution $\left(\tilde{\mathbf{U}}_{h}^{0}, \tilde{\mathbf{U}}_{h}^{1}\right)$ of 81 , which corresponds to some approximate controls $\left(\tilde{\mathbf{v}}_{h}, h^{2} \tilde{\mathbf{g}}_{h}\right)$ defined by 179 with $\tilde{\mathbf{U}}_{h}$ instead of $\mathbf{U}_{h}$. One should then correct this error, and this can be done with small controls using the observability result in Proposition 5. We finally conclude by the uniqueness of controls $\left(\mathbf{v}_{h}, \mathbf{g}_{h}\right)$ that can be written as 179) for some solution $\mathbf{u}_{h}$ of 81 .

\subsection{Numerical Experiments}

In this Section, our goal is to illustrate the convergence results proven above. We focus on the study of the filtering method, the others being very similar.

We first consider the case in which the initial datum to be controlled lies in $L^{2}(0,1) \times H^{-1}(0,1): y^{0}(x)=x^{2}$ for $x \in(0,1 / 2), y^{0}(x)=-(1-x)^{2}$ for $x \in(1 / 2,1)$ and $y^{1} \equiv 0$ (see Figure 11 . 

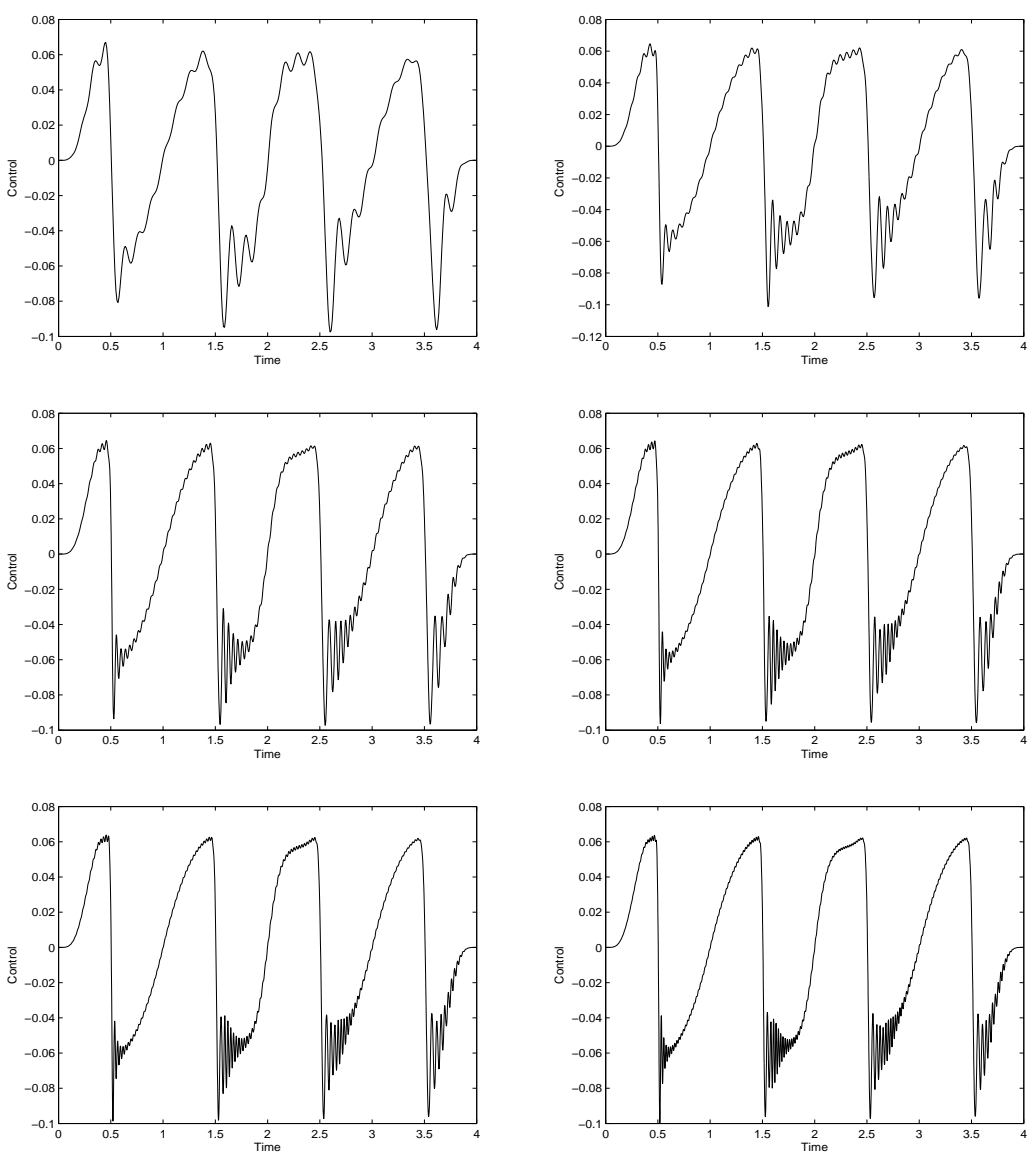

Fig. 12 Discrete controls computed for the initial datum $\left(y^{0}, 0\right)$ with $y^{0}$ as in Figure 11 for different values of $N$, under the CFL condition $\Delta t=0.5 h$, in time $T=4$ and with a filtering parameter $\gamma=1$. From left to right and top to bottom: $N=50, N=100, N=150, N=200, N=250$ and $N=300$.

We then represent in Figure 12 the control functions for various choices of $N$. Note that here, due to the weight function in time, the explicit expression of the control that is given through the minimization of the functional $J$ in (76) is not available anymore.

Here, the wave equation is discretized in time, with a CFL condition $\Delta t=0.5 h$. The filtering parameter is taken to be $\gamma=1$. The function $\eta$ is chosen such that: $\eta=1$ for $t \in(0.4,3.6)$. On $t \in(0,0.4), \eta(t)$ is a polynomial of order 3 so that $\eta(0)=\eta^{\prime}(0)=\eta^{\prime}(1)=0$ and $\eta(0.4)=1$, and we choose it in a similar way in $(3.6,4)$. Of course, $\eta$ is not $C^{\infty}$ smooth but only $C^{1}$, but this would be enough for our purpose. With these choices, the time of control $T=4$ suffices to control the fully discrete dynamics. 
Fig. 13 The initial velocity $y^{1}$ to be controlled.

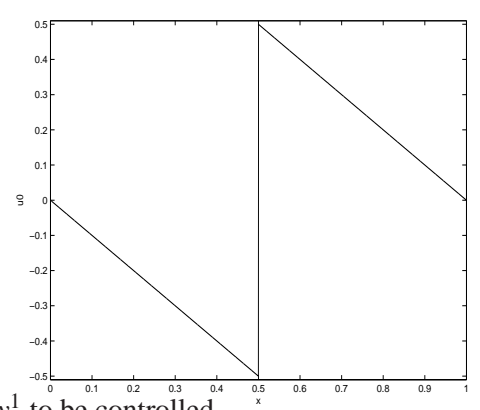

As one can see, the controls in Figure 12 exhibit some kind of Gibbs phenomenon close to the discontinuities of the control.

Let us now present similar numerical results, but for an initial datum to be controlled in $H_{0}^{1}(0,1) \times L^{2}(0,1)$. Now, $\left(y^{0}, y^{1}\right)$ are chosen such that: $y^{0}=0$ and $y^{1}$ is the discontinuous triangular function in Figure 13. The analytic expression of $y^{1}$ is $y^{1}(x)=-x$ for $x \in(0,1 / 2)$ and $y^{1}(x)=1-x$ in $(1 / 2,1)$.

This corresponds to an initial datum to be controlled in $H_{0}^{1}(0,1) \times L^{2}(0,1)$. Therefore, we should expect better convergence properties as before.

We present in Figure 14 the controls computed for that initial data and for several values of $N$. One can see that there, the controls in Figure 14 seem to be smoother than the ones in Figure 12. This is of course consistent with our analysis which states that:

- the smoothness of the continuous control corresponds to the smoothness of the initial datum to be controlled,

- the discrete controls converge towards the continuous one,

and the added regularity of the data being controlled.

To conclude our analysis, we illustrate our results on the rate of convergence of the discrete controls. For that to be done, we take as reference control the one carefully computed for some large reference system size $N_{\text {ref }}$. Using this accurately computed control $V_{N_{r e f}}$, we compute the norm of $V_{N}-V_{N_{\text {ref }}}$ for various $N \leq N_{\text {ref }}$. The rate of convergence of $V_{N}$ towards $V_{N_{\text {ref }}}$ should give a realistic estimate of the convergence rate of the discrete controls towards the continuous one. In log-log scales, this yields Figure 15 .

The linear interpolations of the obtained curves have slope -1.04 when controlling $\left(0, y^{1}\right)$ with $y^{1}$ as in Figure 13 and slope -0.34 when controlling $\left(y^{0}, 0\right)$ with $y^{0}$ as in Figure 11

The fact that, for $\left(0, y^{1}\right)$ with $y^{1}$ as in Figure 13 , the rate is much better than the expected rate $-2 / 3$ predicted by Theorem 20 comes from the fact that the initial datum to be controlled $\left(0, y^{1}\right)$, with $y^{1}$ as in Figure 13 lies not only in $H_{0}^{1}(0,1) \times$ $L^{2}(0,1)$ but in $H_{0}^{s}(0,1) \times H_{0}^{s-1}(0,1)$ for all $s<3 / 2$. This gain of $1 / 2^{-}$derivative with respect of the energy space explain the faster convergence rate as we shall explain below. 

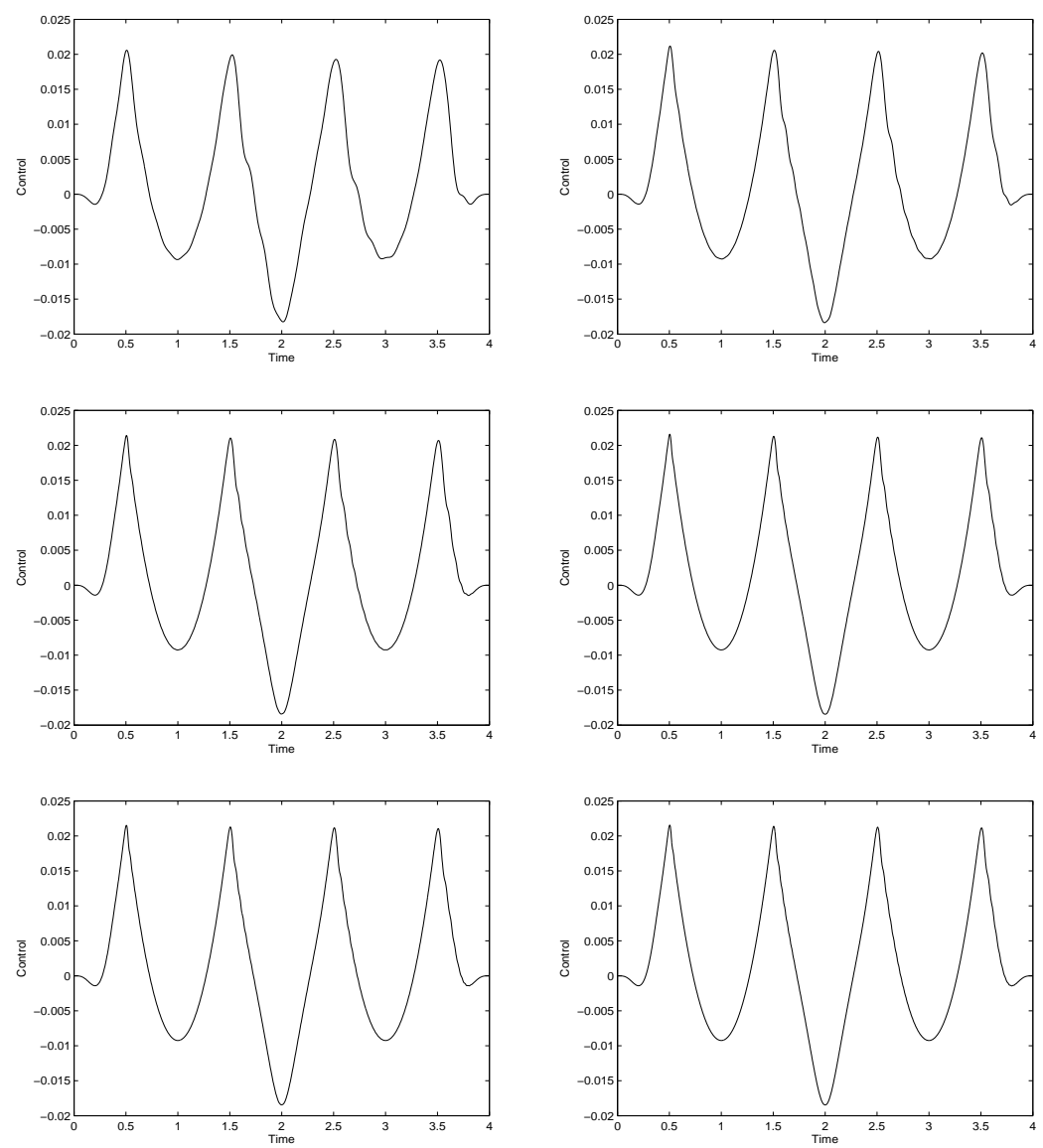

Fig. 14 Discrete controls computed for the initial datum $\left(0, y^{1}\right)$ with $y^{1}$ as in Figure 13 for different values of $N$, under the CFL condition $\Delta t=0.5 h$, in time $T=4$ and with a filtering parameter $\gamma=1$. From left to right and top to bottom: $N=50, N=100, N=150, N=200 N=250$ and $N=300$.

Similarly, $\left(y^{0}, 0\right)$ with $y^{0}$ as in Figure 11 lies not only in $L^{2}(0,1) \times H^{-1}(0,1)$ but also in $H_{0}^{s}(0,1) \times H_{0}^{s-1}(0,1)$ for all $s<1 / 2$, thus explaining why the controls seem to converge with a rate of the order of $1 / 3$.

In fact, the numerical approximations of the controls converge to that of the limit system with rates corresponding to the class of regularity of the initial data under consideration. Actually, following the proofs of [36], if the initial data to be controlled lye in $H_{0}^{s}(0,1) \times H_{0}^{s-1}(0,1)$ for $s \in(0,3 / 2)$ (above the value $s=3 / 2$, more compatibility boundary conditions are required), the convergence rate is of the order of $h^{2 s / 3}$. This is completely consistent with the numerical simulations in Figure 15 since the theory then predicts a convergence rate of order $h^{1 / 3}$ for $s=1 / 2$ and of $h$ for $s=3 / 2$, to be compared with the rates $h^{0.34}$ and $h^{1.04}$ found in Figure 15 For 

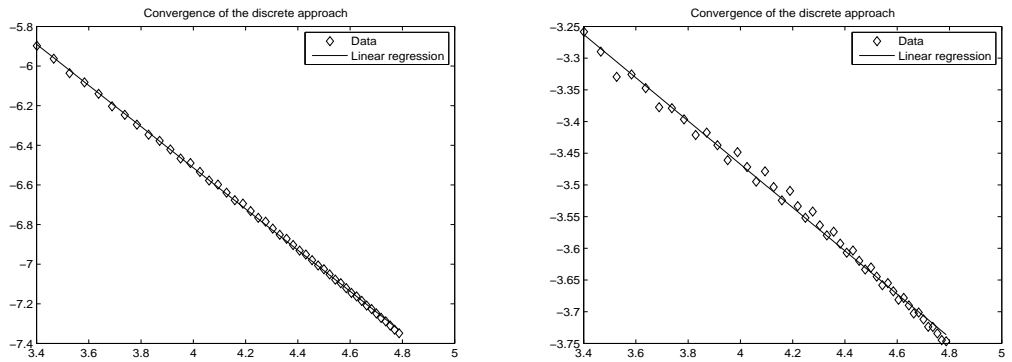

Fig. 15 Graph of $\log \left(\left\|V_{N}-V_{300}\right\|\right)$ as a function of $\log (N)$ for $N \in(30,120)$ : left, for the discrete controls computed for the initial datum $\left(0, y^{1}\right)$ with $y^{1}$ as in Figure 13 the linear interpolant has slope -1.04 ; right, for the discrete controls computed for the initial datum $\left(y^{0}, 0\right)$ with $y^{0}$ as in Figure 11 the linear interpolant has slope -0.34 .

the proof of these more general convergence rates results it suffices, in fact, to prove the analogs of Theorems 19,20 in the spaces of the corresponding regularity and convergence rates.

\section{Further Comments and Open Problems}

\subsection{Further Comments}

1. Time-discrete and fully discrete approximations. In these notes, we have addressed the problem of the convergence of the controls for space semidiscrete approximations of the wave equation as the mesh-size goes to zero. But one can go further and discretize in time these space semidiscrete approximations to obtain fully discrete approximation schemes. This time-discretization adds further spurious high-frequency waves and, consequently, extra difficulties to the fulfillment of the observability inequalities. This is so since the time-discretization process deforms the spectrum and the dispersion relation of the system.

This added numerical dispersion effect has been studied more precisely in [31] for abstract conservative systems (see also [107] for a study of a time discrete and space continuous wave equation) using resolvent type estimates [16, 79, 88]. The interest of the method developed there is that it completely decouples the effects of the space discretization process from the ones originating from the time discretization. Again, the main results can be stated as follows: removing high-frequency solutions, one can get uniform observability properties, where, here, uniformity is referred to space and time discretization parameters. Spurious waves appear at frequencies of the order of $1 /(\Delta t)$, where $\Delta t$ is the time discretization parameter ([31]). On the other hand, the added filtering that the time-discretization processes require can be 
avoided through suitable CFL type conditions on the space and time discretization parameters. These results are sharp, as it has been shown explicitly in [107].

However, the results in [31] do not provide any precise estimate on the time needed to guarantee the uniform observability inequality. This is a drawback of the method developed in [31], which is based on resolvent estimates.

To overcome this drawback, more recently in [36], we have developed a discrete transmutation technique, inspired on previous works, in particular by Miller [80, 79], which establishes a connection between solutions of the time continuous systems and the time-discrete ones. This approach yields explicit estimates on the time needed to guarantee uniform observability results.

The approach developed in Section 6.1 also applies in the context of fully discrete schemes and also yields convergence results for the corresponding discrete controls with explicit convergence rates based on the existing results on the convergence of the fully discrete systems towards the continuous one.

2. Other space discretization methods. In these notes, we have mainly considered the 1-d wave equation discretized using finite differences and we have proved that their observability and controllability properties fail to be uniform as the meshsize parameters tend to zero. This turns out to occur for most numerical methods. In particular, this is also the case for the finite element method, see [53], among others.

However, there are some schemes that enjoy uniform observability properties, but they seem to be very rare. This is the case for instance for the mixed finite element method [41, 17, 18, 28]. For these schemes observability and controllability properties are uniform, without any need of filtering, and the discrete controls converge towards the continuous ones. But this discretization method has an important drawback: Its CFL type condition for stability has the form $\Delta t \leq h^{2}$, where $\Delta t$ is the time discretization parameter. This is in contrast with the above methods which only require $\Delta t \leq h$.

3. Stabilization and discretization. As already noticed in [96], the theory of stabilization and observation/control are strongly linked.

This connection has been made even more precise in [46], showing that the damped wave system

$$
\begin{cases}z_{t t}-\Delta z+\chi_{\omega} z_{t}=0 & \text { in } Q, \\ z=0 & \text { on } \Sigma, \\ z(x, 0)=z^{0}(x), z_{t}(x, 0)=z^{1}(x) & \text { in } \Omega .\end{cases}
$$

is exponentially stable, in the sense that there exist a constant $C$ and a strictly positive constant $\mu>0$ such that for all initial data $\left(z^{0}, z^{1}\right) \in H_{0}^{1}(\Omega) \times L^{2}(\Omega)$ and for all $t \geq 0$,

$$
E(t) \leq C e^{-\mu t} E(0),
$$

if and only if the wave system (64) is observable through $\omega$.

This result can be easily extended to an abstract framework, provided the damping and control operators are bounded. 
In the context of stabilization of waves one often considers boundary damping operators. They turn out to be unbounded perturbations of the conservative semigroup and, therefore, the equivalence of stabilization of the damped system and the observation of the conservative one does not apply. We refer to [1] for partial results in that direction.

Going back to the problem of stabilization by distributed damping as above, and in what concerns the numerical approximation issues, our understanding of the lack of observability for space semidiscrete systems (and fully discrete ones, see Comment \#1 above) suggests that similar pathologies may arise making the decay properties of the corresponding semidiscrete or fully discrete systems not to be uniform. That is indeed the case. As a by byproduct of the lack of uniform observability for (64), the apparently most natural discretizations of (189) are not exponentially uniformly stable, see e.g. [99, 81, 90]. Again, this is due to high-frequency phenomena and spurious solutions coming from the numerical schemes under consideration. One shall then add a numerical viscosity term everywhere in the domain to damp out efficiently these spurious waves. This is the idea that has been developed in [99] for the 1 and 2-d wave equation and then later formalized in a much more general form in [33, 34].

The possible use of two-grid filtering techniques to ensure uniform decay properties is an interesting subject that requires further analysis. Of course, one of the main difficulties is related to the fact that the property of being of two-grid form is not preserved along the dissipative dynamics.

4. Other models. Let us also point out that many control results exist for other type of models, such as Schrödinger equation [67], beam equation [89], where similar ideas as the one presented above can be applied, even if of course, each case presents some specificity and should be handled carefully.

The convergence properties of controls for discrete heat equations has also been developed lately in [70, 61, 30, 8, 9, 10]. The later works [8, 9] are based on Carleman estimates for discrete elliptic operators, which require important technical developments.

\subsection{Open Problems}

Problem 1. Semilinear Wave Equations. We have studied the convergence of the discrete controls for linear wave equations, and we have described the difficulties encountered because of the spurious high-frequency solutions and how to remedy them.

Of course, the same questions arise in the context of semilinear wave equations, even with globally Lipschitz nonlinearities, a case that has been handled for instance in [113]. Most often the nonlinear problems are addressed by means of a fixed point argument together with a careful analysis of the control properties of the linearized system. One of the main difficulties that appears when doing that is to estimate the dependence of the observability constants on the $(t, x)$-depending potentials of the 
linearized the equation. This can be handled using sidewise energy estimates (but this works only in 1-d), multipliers or Carleman estimates [109, 110, 37, 26], thus yielding various constraints on the growth of the non-linearity at infinity for the controllability property to hold. This kind of results guarantees the controllability of the nonlinear system for all initial data in an uniform time.

But one can relax the control problem, analyzing it locally, for small data. Local results, together with exponential convergence ones obtained by means of suitable damping mechanisms, allow showing that, eventually, every initial data can be controlled to zero but on a time that depends on the size of the initial data and that may tend to infinity when the norm of the data tend to infinity. Local results can be proved for nonlinearities growing at infinity in a superlinear manner. When using energy methods, however, one needs to impose growth conditions at infinity. More recently, using dispersive estimates (see [25, 24]), the class of nonlinearities for which this kind of results holds has been extended to cover the range of nonlinearities that can be handled for the well-posedness of the Cauchy problem in the energy space by means of Strichartz inequalities. We refer to the survey article [108] for a discussion of this issue.

The extension of the numerical analysis we have developed and presented in this article to this semilinear setting is a widely open problem. In [115], the adaptation of the two-grid technique to globally Lipschitz nonlinearities is presented, together with some open problems and directions of research.

There is also plenty to be done to adapt the numerical analysis techniques presented here to super-critical exponents since the theory of dispersive estimates for linear discrete waves is also difficult matter in itself. We refer to in [51, 49, 50] for the first results in that direction in the context of Schrödinger equations.

The same problems arise in the context of many other nonlinear PDE, for instance: semilinear Schrödinger equations [62], KdV equations [95], semilinear heat equations [112, 26], etc.

Problem 2. Non-uniform meshes. In applications, one usually deals with nonuniform meshes for finite element methods. But the Fourier analysis methods we have developed here can not be applied in that setting. Roughly speaking, the only existing result in this direction is the one presented in Theorem[13 ([27]), ensuring that, when filtering the high frequencies at the scale $1 / \sqrt{h}$, uniform observability holds. But on uniform meshes, the critical scale is $1 / h$. An in depth analysis is needed in order to explain what is the behavior of numerical waves in this intermediate range for frequencies in between $1 / \sqrt{h}$ and $1 / h$.

The issue is even open in 1-d. For instance, it would worth identifying the class of quasi-uniform meshes for which the $1 / h$ filtering scale suffices.

In this context, the article [28] is worth mentioning: There, it has been proved that, for the mixed finite element method in 1-d on non-uniform meshes, uniform observability properties hold under some mild restrictions on the mesh. This is based on the very nature of the mixed finite element discretization which allows to compute explicitly the spectrum of the discrete equations and then to apply Fourier analysis techniques. 
Note that this issue can also be related to the observability properties of the wave equation with variable coefficients in uniform meshes. For the continuous 1-d wave equation the assumption on the BV regularity of the coefficients is sharp (see [19]). Adapting the numerical analysis results presented in this paper to that setting is a challenging open problem.

Problem 3. Uniform control of the low frequencies. In [77] it has been proved that, in 1-d, for initial data having only a finite number of Fourier components, the discrete controls are uniformly bounded and converge as $h \rightarrow 0$ towards the control of the wave equation. This result has been proved using moment problem techniques. The article [77] provides explicit estimates on the bi-orthogonal functions depending both on the frequency and the mesh-size parameters and in particular yields uniform estimates in the case in which only a finite number of frequencies are involved. This analysis is limited by now to 1-d problems. The extension of this result to multi-dimensional problems, even in the case of the unit square observed from two consecutive boundaries, is a challenging and interesting open problem.

Problem 4. Wigner measures. In [72, 73], Macià adapted Wigner measures to study the propagation of the singularities of waves in a discrete setting on uniform meshes of the whole space (see Problem 2). Roughly speaking, to any sequence of solutions of the discrete wave equation one associates a measure living on the space and frequency variables that is constant along the bicharacteristic flow of the Hamiltonian corresponding to the wave process under consideration. This Wigner measure has some interesting features. In particular, when considering sequences that weakly converge to zero in $L^{2}$, the Wigner measure describes the possible lack of strong convergence very accurately.

But this theory is still to be developed more completely to handle, for instance, boundary conditions and non-uniform meshes or to adapt the notion of polarization introduced in [15] to the discrete setting.

Problem 5. Numerical methods using randomness. When discretizing one dimensional hyperbolic systems of conservation laws, one can use the so-called Glimm's random choice method.

This idea, originally developed in [39], has even been used to prove existence of solutions for one dimensional hyperbolic systems.

A natural question then is the following one: Can we use Glimm's random choice method to obtain convergent sequences of discrete controls? So far, this issue is widely open. The only contribution we are aware of is [22], which states that, for the corresponding discrete 1-d wave equation, with an excellent probability, uniform observability holds. Here, excellent probability means with a probability greater than $\exp \left(-C(T)(\Delta t / h)^{2} /(\Delta t)\right)$, where $\Delta t$ is the time discretization parameter, and $C(T)$ is a strictly positive constant when $T>2$.

But of course, this first result should be further developed, in particular for conservation laws. Also, one could try to extend Glimm's idea to higher dimensions and derive numerical schemes for the 2-d wave equation with some random effects that could help on the obtention of discrete observability properties. 
Problem 6. Inverse Problems. The literature on inverse problems for hyperbolic equations is wide. We refer, for instance, to the works of Bukgheim and Klibanov [13] and the books [12, 58, 56, 59] (and the references therein) for a presentation of the state of the art in that field. For what concerns the acoustic wave equation, we can also refer to the works of [86, 52].

Roughly speaking, the problem is that of determining the properties of a medium by making boundary measurements on the waves propagating in it.

To illustrate the kind of problems that arise in this field and their intrinsic complexity let us consider the example of the $1 \mathrm{~d}$ wave equation in which the velocity of propagation $c$ is a positive unknown constant:

$$
u_{t t}-c^{2} u_{x x}=0, \quad 0<x<1,0<t<T, \quad u(0, t)=u(1, t)=0, \quad 0<t<T .
$$

One could then consider the problem of determining the velocity $c$ out of boundary measurements $u_{x}(1, t)$ for $t \in \mathbb{R}$.

In this continuous setting, using the time periodicity of solutions with time period $2 / c$, one could determine the value of $c$ in terms of the periodicity of the boundary measurement. But, of course, this cannot be applied in the discrete setting since the discrete versions of (190) generate a lot of spurious high-frequency waves that travel at any velocity between 0 and $c$, thus breaking down the periodicity properties of continuous waves.

Hence, even on that simple example, the convergence of the solutions of the discrete inverse problems towards those of the continuous one is not so obvious and very unlikely to hold. Of course, on more intricate examples, the situation will become even worse. Generally speaking, the problem of solving discrete inverse problems and passing to the limit as the mesh-size parameter tends to zero is widely open.

Note that these questions are also of interest for what concerns the so-called Calderón problem, which consists, in the elliptic setting, on identifying the electrical conductivity of a medium by the knowledge of the so-called Dirichlet to Neumann map (or voltage to current map), see [103]. There again, to our knowledge, convergence issues for numerical approximation schemes have not been analyzed.

Problem 7. Unique continuation for discrete waves. For the continuous wave equation in a bounded domain, it is well-known that if the solution vanishes in some open subset during a certain amount of time (which shall be large enough and depends on the whole geometry of the set $\omega$ where the solution vanishes and the domain $\Omega$ where the equation holds), then the solution is identically zero everywhere. For the constant coefficient wave equation this is a consequence of Holmgren's uniqueness theorem, see [48].

Such result is not true for the discrete wave equation, as an explicit counterexample by O. Kavian shows (mentioned in [114]): In the unit square, when discretizing the Laplacian on a uniform grid using the usual 5-points finite-difference discretization, there exists a concentrated eigenvalue, alternating between 1 and -1 on the diagonal, and taking the null value 0 outside. This corresponds to the eigenvalue $4 / h^{2}$, where $h$ is the mesh-size, hence to a very high eigenfunction. Of course, this 
makes the discrete version of the unique continuation property above to be false. However, one could expect this uniqueness property to be true within a class of filtered solutions. This is indeed the case, as it has been recently proved in [9].

But the same can be said about the quantitative versions of the uniqueness theorem above that are by now well known in the continuous setting (see among others, [63, 93, 94, 85]). These results consist in weak observability estimates for the continuous wave equation when no geometric condition is fulfilled.

When no geometric condition is fulfilled, such weak observability estimates for discrete wave equations are so far completely unknown, but we expect this to be reachable using suitable discrete versions of the Carleman inequalities, the preliminary results by [9] and the so-called Fourier-Bros-Iagoniltzer transform [94, 85].

Problem 8. Waves on networks. Several important applications require the understanding of the propagation of waves into networks, and their control theoretical properties. Even in the continuous setting, this question is intricate since the propagation of the waves in a network depend strongly on its geometrical and topological properties. In particular, when the network includes a closed loop, some resonant effects may appear. We refer to [23] (and to the references therein) for a precise description of the state of the art in this field, updated in the recent survey [116].

Hence, when discretizing these models, understanding the propagation, observation and control properties of discrete waves propagate into networks, becomes a complex topic that is widely open. Some preliminary results have been obtained in [11] on a star shaped network of three strings controlled from the exterior nodes. But there is still an important gap between the understanding of the observability properties of the waves on networks in the discrete and continuous frameworks.

Problem 9. Hybrid parabolic/hyperbolic systems. In these notes we focused on the classical wave equation and its semidiscrete approximation schemes, but in many applications the relevant models are much more complex.

A classical example is given by the system of linear thermoelasticity, whose nullcontrollability properties have been derived in [65]. This system is composed of one parabolic type equation coupled with an hyperbolic one. In [65], it is proved that the system of linear thermoelasticity is null-controllable when the Geometric Control Condition is satisfied, which of course comes from the hyperbolic nature of the underlying wave equation.

When discretizing such equations, in view of the results developed above, it is natural to expect that the discrete controllability properties may fail to be uniform. But this should be discussed more precisely, because of the coupling with the parabolic component that may strongly influence the dynamics.

Acknowledgements. When preparing the last version of this manuscript we were supported by Alejandro Maas Jr., internship student from the Universidad Técnica Federico Santa María (UTFSM), Chile, visiting BCAM for two months early 2011. He made an important contribution to improve our plots and also to run the numerical experiments we present here. We express our gratitude to him for his efficient and friendly help. 


\section{References}

1. K. Ammari and M. Tucsnak. Stabilization of second order evolution equations by a class of unbounded feedbacks. ESAIM Control Optim. Calc. Var., 6:361-386 (electronic), 2001.

2. M. Asch and G. Lebeau. Geometrical aspects of exact boundary controllability for the wave equation-a numerical study. ESAIM Control Optim. Calc. Var., 3:163-212 (electronic), 1998.

3. D. Auroux and J. Blum. Back and forth nudging algorithm for data assimilation problems. C. R. Math. Acad. Sci. Paris, 340(12):873-878, 2005.

4. G. A. Baker and J. H. Bramble. Semidiscrete and single step fully discrete approximations for second order hyperbolic equations. RAIRO Anal. Numér., 13(2):75-100, 1979.

5. C. Bardos, F. Bourquin, and G. Lebeau. Calcul de dérivées normales et méthode de Galerkin appliquée au problème de contrôlabilité exacte. C. R. Acad. Sci. Paris Sér. I Math., 313(11):757-760, 1991.

6. C. Bardos, G. Lebeau, and J. Rauch. Sharp sufficient conditions for the observation, control and stabilization of waves from the boundary. SIAM J. Control and Optim., 30(5):1024-1065, 1992.

7. T. Z. Boulmezaoud and J. M. Urquiza. On the eigenvalues of the spectral second order differentiation operator and application to the boundary observability of the wave equation. J. Sci. Comput., 31(3):307-345, 2007.

8. F. Boyer, F. Hubert, and J. Le Rousseau. Discrete Carleman estimates for elliptic operators and uniform controllability of semi-discretized parabolic equations. J. Math. Pures Appl. (9), 93(3):240-276, 2010.

9. F. Boyer, F. Hubert, and J. Le Rousseau. Discrete carleman estimates for elliptic operators in arbitrary dimension and applications,. SIAM J. Control Optim., 48:5357-5397, 2010.

10. F. Boyer, F. Hubert, and J. Le Rousseau. Uniform null-controllability properties for space/time-discretized parabolic equations. Numer. Math., to appear.

11. U. Brauer and G. Leugering. On boundary observability estimates for semi-discretizations of a dynamic network of elastic strings. Control Cybernet., 28(3):421-447, 1999. Recent advances in control of PDEs.

12. A. L. Bughgeim. Volterra equations and inverse problems. Inverse and Ill-posed Problems Series. VSP, Utrecht, 1999.

13. A. L. Bukhgerm and M. V. Klibanov. Uniqueness in the large of a class of multidimensional inverse problems. Dokl. Akad. Nauk SSSR, 260(2):269-272, 1981.

14. N. Burq and P. Gérard. Condition nécessaire et suffisante pour la contrôlabilité exacte des ondes. C. R. Acad. Sci. Paris Sér. I Math., 325(7):749-752, 1997.

15. N. Burq and G. Lebeau. Mesures de défaut de compacité, application au système de Lamé. Ann. Sci. École Norm. Sup. (4), 34(6):817-870, 2001.

16. N. Burq and M. Zworski. Geometric control in the presence of a black box. J. Amer. Math. Soc., 17(2):443-471 (electronic), 2004

17. C. Castro and S. Micu. Boundary controllability of a linear semi-discrete 1-d wave equation derived from a mixed finite element method. Numer. Math., 102(3):413-462, 2006.

18. C. Castro, S. Micu, and A. Münch. Numerical approximation of the boundary control for the wave equation with mixed finite elements in a square. IMA J. Numer. Anal., 28(1):186-214, 2008.

19. C. Castro and E. Zuazua. Concentration and lack of observability of waves in highly heterogeneous media. Arch. Ration. Mech. Anal., 164(1):39-72, 2002.

20. N. Cîndae, S. Micu, and M. Tucsnak. An approximation method for exact controls of vibrating systems. Preprint, 2010.

21. G. C. Cohen. Higher-order numerical methods for transient wave equations. Scientific Computation. Springer-Verlag, Berlin, 2002. With a foreword by R. Glowinski.

22. J.-M. Coron, S. Ervedoza, and O. Glass. Uniform observability estimates for the 1-d discretized wave equation and the random choice method. Comptes Rendus Mathematique, 347(9-10):505 - 510, 2009. 
23. R. Dáger and E. Zuazua. Wave propagation, observation and control in 1-d flexible multistructures, volume 50 of Mathématiques \& Applications (Berlin). Springer-Verlag, Berlin, 2006.

24. B. Dehman and G. Lebeau. Analysis of the HUM control operator and exact controllability for semilinear waves in uniform time. SIAM J. Control and Optim., 48(2):521-550, 2009.

25. B. Dehman, G. Lebeau, and E. Zuazua. Stabilization and control for the subcritical semilinear wave equation. Ann. Sci. École Norm. Sup. (4), 36(4):525-551, 2003.

26. T. Duyckaerts, X. Zhang, and E. Zuazua. On the optimality of the observability inequalities for parabolic and hyperbolic systems with potentials. Ann. Inst. H. Poincaré Anal. Non Linéaire, 25(1):1-41, 2008.

27. S. Ervedoza. Spectral conditions for admissibility and observability of wave systems: applications to finite element schemes. Numer. Math., 113(3):377-415, 2009.

28. S. Ervedoza. Observability properties of a semi-discrete 1D wave equation derived from a mixed finite element method on nonuniform meshes. ESAIM Control Optim. Calc. Var., 16(2):298-326, 2010.

29. S. Ervedoza. Admissibility and observability for Schrödinger systems: Applications to finite element approximation schemes. Asymptot. Anal., 71(1-2):1-32, 2011.

30. S. Ervedoza and J. Valein. On the observability of abstract time-discrete linear parabolic equations. Rev. Mat. Complut., 23(1):163-190, 2010.

31. S. Ervedoza, C. Zheng, and E. Zuazua. On the observability of time-discrete conservative linear systems. J. Funct. Anal., 254(12):3037-3078, June 2008.

32. S. Ervedoza and E. Zuazua. Analysis of the hum control operator for the computation of exact controls for conservative systems. Work in progress.

33. S. Ervedoza and E. Zuazua. Uniform exponential decay for viscous damped systems. In Advances in phase space analysis of partial differential equations, volume 78 of Progr. Nonlinear Differential Equations Appl., pages 95-112. Birkhäuser Boston Inc., Boston, MA, 2009.

34. S. Ervedoza and E. Zuazua. Uniformly exponentially stable approximations for a class of damped systems. J. Math. Pures Appl., 91:20-48, 2009.

35. S. Ervedoza and E. Zuazua. A systematic method for building smooth controls for smooth data. Discrete Contin. Dyn. Syst. Ser. B, 14(4):1375-1401, 2010.

36. S. Ervedoza and E. Zuazua. Propagation, observation and numerical approximations of waves. Book in preparation.

37. X. Fu, J. Yong, and X. Zhang. Exact controllability for multidimensional semilinear hyperbolic equations. SIAM J. Control Optim., 46(5):1578-1614 (electronic), 2007.

38. P. Gérard. Microlocal defect measures. Comm. Partial Differential Equations, 16(11):17611794, 1991.

39. J. Glimm. Solutions in the large for nonlinear hyperbolic systems of equations. Comm. Pure Appl. Math., 18:697-715, 1965.

40. R. Glowinski. Ensuring well-posedness by analogy: Stokes problem and boundary control for the wave equation. J. Comput. Phys., 103(2):189-221, 1992.

41. R. Glowinski, W. Kinton, and M. F. Wheeler. A mixed finite element formulation for the boundary controllability of the wave equation. Internat. J. Numer. Methods Engrg., 27(3):623-635, 1989.

42. R. Glowinski and C. H. Li. On the numerical implementation of the Hilbert uniqueness method for the exact boundary controllability of the wave equation. C. R. Acad. Sci. Paris Sér. I Math., 311(2):135-142, 1990.

43. R. Glowinski, C. H. Li, and J.-L. Lions. A numerical approach to the exact boundary controllability of the wave equation. I. Dirichlet controls: description of the numerical methods. Japan J. Appl. Math., 7(1):1-76, 1990.

44. R. Glowinski, J.-L. Lions, and J. He. Exact and approximate controllability for distributed parameter systems, volume 117 of Encyclopedia of Mathematics and its Applications. Cambridge University Press, Cambridge, 2008. A numerical approach.

45. P. Grisvard. Contrôlabilité exacte des solutions de l'équation des ondes en présence de singularités. J. Math. Pures Appl. (9), 68(2):215-259, 1989. 
46. A. Haraux. Une remarque sur la stabilisation de certains systèmes du deuxième ordre en temps. Port. Math., 46(3):245-258, 1989.

47. L. F. Ho. Observabilité frontière de l'équation des ondes. C. R. Acad. Sci. Paris Sér. I Math., 302(12):443-446, 1986.

48. L. Hörmander. Linear partial differential operators. Die Grundlehren der mathematischen Wissenschaften, Bd. 116. Academic Press Inc., Publishers, New York, 1963.

49. L. I. Ignat and E. Zuazua. Dispersive properties of a viscous numerical scheme for the Schrödinger equation. C. R. Math. Acad. Sci. Paris, 340(7):529-534, 2005.

50. L. I. Ignat and E. Zuazua. A two-grid approximation scheme for nonlinear Schrödinger equations: dispersive properties and convergence. C. R. Math. Acad. Sci. Paris, 341(6):381386, 2005.

51. L. I. Ignat and E. Zuazua. Numerical dispersive schemes for the nonlinear Schrödinger equation. SIAM J. Numer. Anal., 47(2):1366-1390, 2009.

52. O. Y. Imanuvilov and M. Yamamoto. Determination of a coefficient in an acoustic equation with a single measurement. Inverse Problems, 19(1):157-171, 2003.

53. J.A. Infante and E. Zuazua. Boundary observability for the space semi discretizations of the 1-d wave equation. Math. Model. Num. Ann., 33:407-438, 1999.

54. A. E. Ingham. Some trigonometrical inequalities with applications to the theory of series. Math. Z., 41(1):367-379, 1936.

55. E. Isaacson and H. B. Keller. Analysis of numerical methods. John Wiley \& Sons Inc., New York, 1966.

56. V. Isakov. Inverse problems for partial differential equations, volume 127 of Applied Mathematical Sciences. Springer, New York, second edition, 2006.

57. J. Klamka. Controllability of dynamical systems, volume 48 of Mathematics and its Applications (East European Series). Kluwer Academic Publishers Group, Dordrecht, 1991.

58. M. V. Klibanov. Inverse problems and Carleman estimates. Inverse Problems, 8(4):575-596, 1992.

59. M. V. Klibanov and A. Timonov. Carleman estimates for coefficient inverse problems and numerical applications. Inverse and Ill-posed Problems Series. VSP, Utrecht, 2004.

60. V. Komornik. A new method of exact controllability in short time and applications. Ann. Fac. Sci. Toulouse Math. (5), 10(3):415-464, 1989.

61. S. Labbé and E. Trélat. Uniform controllability of semidiscrete approximations of parabolic control systems. Systems Control Lett., 55(7):597-609, 2006.

62. C. Laurent. Global controllability and stabilization for the nonlinear Schrödinger equation on some compact manifolds of dimension 3. SIAM J. Math. Anal., 42(2):785-832, 2010.

63. G. Lebeau. Contrôle analytique. I. Estimations a priori. Duke Math. J., 68(1):1-30, 1992.

64. G. Lebeau and M. Nodet. Experimental study of the HUM control operator for linear waves. Experiment. Math., 19(1):93-120, 2010.

65. G. Lebeau and E. Zuazua. Null-controllability of a system of linear thermoelasticity. Arch. Rational Mech. Anal., 141(4):297-329, 1998.

66. E. B. Lee and L. Markus. Foundations of optimal control theory. Robert E. Krieger Publishing Co. Inc., Melbourne, FL, second edition, 1986.

67. L. León and E. Zuazua. Boundary controllability of the finite-difference space semidiscretizations of the beam equation. ESAIM Control Optim. Calc. Var, 8:827-862 (electronic), 2002. A tribute to J. L. Lions.

68. J.-L. Lions. Contrôlabilité exacte, Stabilisation et Perturbations de Systèmes Distribués. Tome 1. Contrôlabilité exacte, volume RMA 8. Masson, 1988.

69. J.-L. Lions. Exact controllability, stabilization and perturbations for distributed systems. SIAM Rev., 30(1):1-68, 1988.

70. A. López and E. Zuazua. Some new results related to the null controllability of the 1-d heat equation. In Séminaire sur les Équations aux Dérivées Partielles, 1997-1998, pages Exp. No. VIII, 22. École Polytech., Palaiseau, 1998.

71. P. Loreti and M. Mehrenberger. An ingham type proof for a two-grid observability theorem. ESAIM: COCV, 14(3):604-631, 2008. 
72. F. Macià. The effect of group velocity in the numerical analysis of control problems for the wave equation. In Mathematical and numerical aspects of wave propagation-WAVES 2003, pages 195-200. Springer, Berlin, 2003.

73. F. Macià. Wigner measures in the discrete setting: high-frequency analysis of sampling and reconstruction operators. SIAM J. Math. Anal., 36(2):347-383 (electronic), 2004.

74. F. Macià and E. Zuazua. On the lack of observability for wave equations: a Gaussian beam approach. Asymptot. Anal., 32(1):1-26, 2002.

75. A. Marica. Propagation and dispersive properties for the discontinuous Galerkin and higher order finite element approximations of the wave and Schrödinger equations, $P h D$ Thesis, Universidad Autónoma de Madrid, 2010.

76. A. Marica and E. Zuazua. Localized solutions for the finite difference semi-discretization of the wave equation. C. R. Math. Acad. Sci. Paris, 348(11-12):647-652, 2010.

77. S. Micu. Uniform boundary controllability of a semi-discrete 1-D wave equation. Numer. Math., 91(4):723-768, 2002.

78. S. Micu and E. Zuazua. An introduction to the controllability of partial differential equations. Collection Travaux en Cours Hermannin "Quelques questions de théorie du contrôle”, pages 67-150, Sari, T., ed., 2005.

79. L. Miller. Controllability cost of conservative systems: resolvent condition and transmutation. J. Funct. Anal., 218(2):425-444, 2005.

80. L. Miller. The control transmutation method and the cost of fast controls. SIAM J. Control Optim., 45(2):762-772 (electronic), 2006.

81. A. Münch and A. F. Pazoto. Uniform stabilization of a viscous numerical approximation for a locally damped wave equation. ESAIM Control Optim. Calc. Var., 13(2):265-293 (electronic), 2007.

82. M. Negreanu, A.-M. Matache, and C. Schwab. Wavelet filtering for exact controllability of the wave equation. SIAM J. Sci. Comput., 28(5):1851-1885 (electronic), 2006.

83. M. Negreanu and E. Zuazua. Convergence of a multigrid method for the controllability of a 1-d wave equation. C. R. Math. Acad. Sci. Paris, 338(5):413-418, 2004.

84. A. Osses. A rotated multiplier applied to the controllability of waves, elasticity, and tangential Stokes control. SIAM J. Control Optim., 40(3):777-800 (electronic), 2001.

85. K. D. Phung. Waves, damped wave and observation. In Ta-Tsien Li, Yue-Jun Peng, and BoPeng Rao, editors, Some Problems on Nonlinear Hyperbolic Equations and Applications, Series in Contemporary Applied Mathematics CAM 15, 2010.

86. J.-P. Puel and M. Yamamoto. On a global estimate in a linear inverse hyperbolic problem. Inverse Problems, 12(6):995-1002, 1996.

87. J. V. Ralston. Solutions of the wave equation with localized energy. Comm. Pure Appl. Math., 22:807-823, 1969.

88. K. Ramdani, T. Takahashi, G. Tenenbaum, and M. Tucsnak. A spectral approach for the exact observability of infinite-dimensional systems with skew-adjoint generator. J. Funct. Anal., 226(1):193-229, 2005.

89. K. Ramdani, T. Takahashi, and M. Tucsnak. Semi-discrétisation en espace du problème de la stabilisation interne de l'équation des poutres. ESAIM: Proceedings, 18:48-56, 2007.

90. K. Ramdani, T. Takahashi, and M. Tucsnak. Uniformly exponentially stable approximations for a class of second order evolution equations-application to LQR problems. ESAIM Control Optim. Calc. Var., 13(3):503-527, 2007.

91. J. Rauch. On convergence of the finite element method for the wave equation. SIAM J. Numer. Anal., 22(2):245-249, 1985.

92. P.-A. Raviart and J.-M. Thomas. Introduction à l'analyse numérique des équations aux dérivées partielles. Collection Mathématiques Appliquées pour la Maitrise. [Collection of Applied Mathematics for the Master's Degree]. Masson, Paris, 1983.

93. L. Robbiano. Théorème d'unicité adapté au contrôle des solutions des problèmes hyperboliques. Comm. Partial Differential Equations, 16(4-5):789-800, 1991.

94. L. Robbiano. Fonction de coût et contrôle des solutions des équations hyperboliques. Asymptotic Anal., 10(2):95-115, 1995. 
95. L. Rosier and B.-Y. Zhang. Control and stabilization of the Korteweg-de Vries equation: recent progresses. J. Syst. Sci. Complex., 22(4):647-682, 2009.

96. D. L. Russell. Controllability and stabilizability theory for linear partial differential equations: recent progress and open questions. SIAM Rev., 20(4):639-739, 1978.

97. T. I. Seidman and J. Yong. How violent are fast controls? II. Math. Control Signals Systems, 9(4):327-340, 1996.

98. E. D. Sontag. Mathematical control theory, volume 6 of Texts in Applied Mathematics. Springer-Verlag, New York, second edition, 1998. Deterministic finite-dimensional systems.

99. L. R. Tcheugoué Tebou and E. Zuazua. Uniform boundary stabilization of the finite difference space discretization of the $1 d$ wave equation. Adv. Comput. Math., 26(1-3):337-365, 2007.

100. L.R. Tcheugoué Tébou and E. Zuazua. Uniform exponential long time decay for the space semi-discretization of a locally damped wave equation via an artificial numerical viscosity. Numer. Math., 95(3):563-598, 2003.

101. L. N. Trefethen. Group velocity in finite difference schemes. SIAM Rev., 24(2):113-136, 1982.

102. M. Tucsnak and G. Weiss. Observation and Control for Operator Semigroups, volume XI of Birkäuser Advanced Texts. Springer, 2009.

103. G. Uhlmann. Developments in inverse problems since Calderón's foundational paper. In Harmonic analysis and partial differential equations (Chicago, IL, 1996), Chicago Lectures in Math., pages 295-345. Univ. Chicago Press, Chicago, IL, 1999.

104. R. Vichnevetsky and J. B. Bowles. Fourier analysis of numerical approximations of hyperbolic equations, volume 5 of SIAM Studies in Applied Mathematics. SIAM, Philadelphia, PA., 1982. With a foreword by G. Birkhoff.

105. R. M. Young. An introduction to nonharmonic Fourier series. Academic Press Inc., San Diego, CA, first edition, 2001.

106. X. Zhang. Explicit observability estimate for the wave equation with potential and its application. R. Soc. Lond. Proc. Ser. A Math. Phys. Eng. Sci., 456(1997):1101-1115, 2000.

107. X. Zhang, C. Zheng, and E. Zuazua. Exact controllability of the time discrete wave equation. Discrete and Continuous Dynamical Systems, 2007.

108. X. Zhang and E. Zuazua. Exact controllability of the semi-linear wave equation. In Princeton University Press, editor, Unsolved problems in mathematical systems and control theory, pages 173-178, 2004.

109. E. Zuazua. Exact controllability for the semilinear wave equation. J. Math. Pures Appl. (9), 69(1):1-31, 1990.

110. E. Zuazua. Exact controllability for semilinear wave equations in one space dimension. Ann. Inst. H. Poincaré Anal. Non Linéaire, 10(1):109-129, 1993.

111. E. Zuazua. Boundary observability for the finite-difference space semi-discretizations of the 2-D wave equation in the square. J. Math. Pures Appl. (9), 78(5):523-563, 1999.

112. E. Zuazua. Some results and open problems on the controllability of linear and semilinear heat equations. In Carleman estimates and applications to uniqueness and control theory (Cortona, 1999), volume 46 of Progr. Nonlinear Differential Equations Appl., pages 191211. Birkhäuser Boston, Boston, MA, 2001.

113. E. Zuazua. Controllability of partial differential equations and its semi-discrete approximations. Discrete Contin. Dyn. Syst., 8(2):469-513, 2002. Current developments in partial differential equations (Temuco, 1999).

114. E. Zuazua. Propagation, observation, and control of waves approximated by finite difference methods. SIAM Rev., 47(2):197-243 (electronic), 2005.

115. E. Zuazua. Control and numerical approximation of the wave and heat equations. In International Congress of Mathematicians. Vol. III, pages 1389-1417. Eur. Math. Soc., Zürich, 2006.

116. E. Zuazua. Control and stabilization of waves on $1-d$ networks. In B. Piccoli and M. Rascle, editors, Traffic flow on networks, CIME Subseries. Springer Verlag, to appear. 